Argonne National Laboratory, a U.S. Department of Energy Office of Science laboratory, is operated by The University of Chicago under contract W-31-109-Eng-38.

\title{
DISCLAIMER
}

This report was prepared as an account of work sponsored by an agency of the United States Government. Neither the United States Government nor any agency thereof, nor The University of Chicago, nor any of their employees or officers, makes any warranty, express or implied, or assumes any legal liability or responsibility for the accuracy, completeness, or usefulness of any information, apparatus, product, or process disclosed, or represents that its use would not infringe privately owned rights. Reference herein to any specific commercial product, process, or service by trade name, trademark, manufacturer, or otherwise, does not necessarily constitute or imply its endorsement, recommendation, or favoring by the United States Government or any agency thereof. The views and opinions of document authors expressed herein do not necessarily state or reflect those of the United States Government or any agency thereof, Argonne National Laboratory, or The University of Chicago. 
ANL-04/19

ARGONNE NATIONAL LABORATORY

9700 South Cass Avenue

Argonne, Illinois 60439

\section{PRACTICAL SUPERCONDUCTOR DEVELOPMENT FOR ELECTRICAL POWER APPLICATIONS}

ANNUAL REPORT FOR FY 2003

U. Balachandran

Contributors:

R. Baurceanu

R. E. Koritala

J. P. Singh

H. Claus*

M. Li

S. Srinivasan

S. E. Dorris

B. $\mathrm{Ma}$

Y. Tang*

J. E. Emerson

V. A. Maroni**

K. Uprety

R. A. Erck

R. L. McDaniel

B. Veal ${ }^{*}$

B. L. Fisher

M. Mika

V. Vlasko-Vlasov*

K. E. Gray*

D. J. Miller*

U. Welp*

J. R. Hull

J. J. Picciolo

Energy Technology Division

October 2004

Work Supported by

U. S. DEPARTMENT OF ENERGY

Office of Electric Transmission and Distribution

*Materials Science Division

${ }^{* *}$ Chemical Engineering Division 


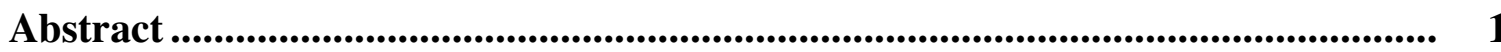

1 Introduction................................................................................................................ 1

2 Technical Progress in 2002-2003............................................................................ 1

2.1) Y-Ba-Cu-O Coated Conductors.......................................................................... 2

2.1.1) Conductors with Standard ISD-MgO Architecture .............................. 2

2.1.1a) ISD-MgO Process...................................................................... 2

2.1.1b) Performance of Standard ISD-MgO Conductors ................................ 4

2.1.1c) Texture Development/Crystallography of Standard ISD Conductors... 7

2.1.2) Conductors with $\mathrm{SrRuO}_{3}(\mathrm{SRO})$-Buffered ISD MgO Architecture.... 11

2.1.2a) Initial Study of Feasibility ........................................................... 11

2.1.2b) Study of SRO-Buffered MgO Single-Crystal Substrates ..................... 15

2.1.2c) SRO-Buffered ISD-MgO Substrates ................................................ 23

2.1.3) Direct Deposition of YBCO on Ag Alloy Substrates ............................. 27

2.1.4) Raman Microscopy of Coated Conductors............................................. 30

2.1.4a) Studies Probing Applicability of Raman Microscopy …….................. 30

2.1.4b) Basic Features of Raman Microscopy Relevant to YBCO................... 40

2.1.4c) Raman Microscopy for Examining YBCO Precursor Evolution.......... 43

2.1.5) Oxygen Doping of YBCO Grain Boundaries.......................................... 53

2.1.6) Mechanical Properties of Coated Conductors........................................ 60

2.1.6a) Strain Tolerance of YBCO in ISD-Based Conductors ........................ 60

2.1.6b) Residual Stress Evaluation.......................................................... 62

\section{2) Bi-Sr-Ca-Cu-O Conductor}

Modifications to Thermal Slide Heat Treatment of Bi-2223 Conductors ... 62

References................................................................................................................... 65

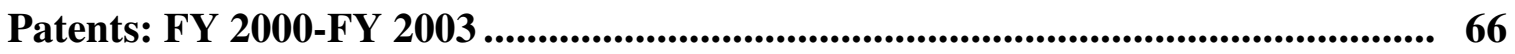

Publications: FY 2003 _....................................................................................................... 67 


\section{Figures}

1. Transport measurements $(77 \mathrm{~K})$ for sample with standard ISD architecture

2. Field-dependence of transport $J_{C}(77 \mathrm{~K})$ for sample with standard ISD architecture.

3. Relative transport $\mathrm{J}_{\mathrm{c}}(77 \mathrm{~K}, 1 \mathrm{~T}$ applied field) for standard ISD architecture vs. $\xi$, the angle between film surface and applied field.

4. Full-width-at-half-maximum of $\mathrm{MgO}(002) \phi$-scans vs. position in 1-m-long ISD $\mathrm{MgO}$ tape.

5. Electron diffraction patterns collected from various locations in ISD-MgO template layer

6. Full-width at half-maximum of electron diffraction reflection as function of position in ISD-MgO template layer.

7. Electron diffraction patterns from ISD-MgO and $\mathrm{HE}-\mathrm{MgO}$ layers showing enhanced texture in HE layer.

8. Bright-field TEM image from "standard" ISD conductor and corresponding electron diffraction pattern used to determine orientation relationships

9. $\mathrm{CeO}_{2}[211]$ and $\mathrm{YBCO}[001]$ reciprocal space representations showing $\mathrm{CeO}_{2}(001) / / \mathrm{YBCO}(010)$ orientation relationship minimizes elastic interactions

10. Superconducting transition for $\mathrm{YBCO}$ on SRO-buffered $\mathrm{MgO}$ single crystal

11. Current/voltage curve for $\mathrm{YBCO}$ on SRO-buffered $\mathrm{MgO}$ single crystal

12. Plan-view SEM images of SRO buffer layer and YBCO film on SRO-buffered ISD $\mathrm{MgO}$ substrate

13. X-ray pole figures $[\mathrm{MgO}(002), \mathrm{SRO}(020), \mathrm{YBCO}(005)$, and YBCO (103)] measured from YBCO deposited on SRO-buffered ISD-MgO substrate

14. Current/voltage curve for YBCO on SRO-buffered ISD-MgO on $\mathrm{HC}$ substrate.

15. $\mathrm{SRO}(220) \phi$-scan and $\mathrm{SRO}(020) \omega$-scan for SRO deposited by $\mathrm{PLD}$ at $770^{\circ} \mathrm{C}$ on $\mathrm{MgO}$ single crystal substrate.

16. Full-width-at-half-maximum of $\mathrm{YBCO}(113)$-scan and YBCO(003) $\omega$-scan vs. temperature of YBCO deposition on SRO-buffered $\mathrm{MgO}$ single crystal substrates. 
17. YBCO(113) $\phi$-scan and $\mathrm{YBCO}(003) \omega$-scan for YBCO deposited at $770^{\circ} \mathrm{C}$ on SRO-buffered $\mathrm{MgO}$ single crystal substrate.

18. Critical temperature, $T_{C}$, and superconducting transition width, $\triangle \mathrm{T}$, for $\mathrm{YBCO}$ on SRO-buffered single crystal $\mathrm{MgO}$ substrates vs. temperature of $\mathrm{YBCO}$ deposition

19. Superconducting transition, $\triangle \mathrm{T}$, for $\mathrm{YBCO}$ deposited at $770^{\circ} \mathrm{C}$ on SRO-buffered $\mathrm{MgO}$ single crystal substrate

20. Transport $\mathrm{J}_{\mathrm{C}}$ for $\mathrm{YBCO}$ films on SRO-buffered single crystal $\mathrm{MgO}$ substrates vs. temperature of YBCO deposition.

21. Secondary electron images of SRO deposited by PLD on $\mathrm{MgO}$ single crystal substrates at $770^{\circ} \mathrm{C}$ and $790^{\circ} \mathrm{C}$

22. Secondary electron images of $\mathrm{YBCO}$ deposited by PLD at $770^{\circ} \mathrm{C}$ on SRO-buffered $\mathrm{MgO}$ single crystal substrate

23. Raman spectra of YBCO deposited on SRO-buffered $\mathrm{MgO}$ single crystal substrate at 770 and $790^{\circ} \mathrm{C}$

24. X- ray diffraction pattern of YBCO on SRO-buffered ISD-MgO on $\mathrm{HC}$ substrate.

25. $\mathrm{SRO}(020)$ and $\mathrm{MgO}(200) \phi$-scans from SRO-buffered ISD-MgO on $\mathrm{HC}$ substrate.

26. $\mathrm{YBCO}(103) \phi$-scan for $\mathrm{YBCO}$ on SRO-buffered ISD-MgO on HC substrate.

27. Transport $\mathrm{J}_{\mathrm{C}}$ of $\mathrm{YBCO}$ on SRO-buffered ISD-MgO on HC substrate

28. Superconducting transition of $\mathrm{YBCO}$ on SRO-buffered ISD$\mathrm{MgO}$ on HC substrate.

29. (111) and (220) pole figures from Ag-0.2 at.\% Cu substrates with $\{110\}<110>$ and $\{110\}<112>$ textures

30. Thermal faceting and grain boundary grooving in $\mathrm{Ag}-\mathrm{Cu}$ alloy substrate after exposure to PLD characteristic temperatures

31. SEM images showing particles and voids in YBCO deposited on Ag-Cu substrates that had been recrystallized in oxygencontaining atmosphere

32. SEM image of Ag-Cu substrate after it was annealed at $780^{\circ} \mathrm{C}$ in $1 \mathrm{~atm}$ Ar.

33. Raman spectra from three thicknesses of EuBCO deposited by PLD on single-crystal La-Sr-Al-Ta-O substrates 
34. Raman spectra from 0.19 - and $1.80-\mu \mathrm{m}$-thick YBCO films deposited by PLD on single-crystal $\mathrm{SrTiO}_{3}$, recorded with laser excitation wavelengths of 633 and $476 \mathrm{~nm}$

35. Raman spectra from YBCO films exhibiting $470 \mathrm{~cm}^{-1}$ mode indicative of tetragonal YBCO: (1) YBCO (by PLD)/ $\mathrm{SmBCO}$ (by $\mathrm{PLD}) / \mathrm{YBCO}$ (by $\mathrm{PLD}) / \mathrm{CeO}_{2} / \mathrm{YSZ} / \mathrm{HC}$, (2) YBCO(by $\mathrm{PLD}) / \mathrm{CeO}_{2} / \mathrm{YSZ} / \mathrm{CeO}_{2} / \mathrm{Ni}-\mathrm{RABiTS}$, (3) tetragonal YBCO bulk specimen (melt-processed/ textured)

36. Averaged Raman spectra of YBCO deposited by PLD with thickness of $0.19,0.30,0.44,0.88,1.30$, and $1.80 \mu \mathrm{m}$ on singlecrystal $\mathrm{SrTiO}_{3}$ substrates and thickness of $0.74 \mu \mathrm{m}$ on singlecrystal $\mathrm{LaAlO}_{3}$

37. Raman microprobe texture map for 0.19 - and $1.80-\mu \mathrm{m}$-thick YBCO films deposited by PLD on single crystal $\mathrm{SrTiO}_{3}$

38. Raman spectra of $\mathrm{YBCO}$ produced by $\mathrm{BaF}_{2}$-type ex situ process (at ORNL) on IBAD substrates produced at LANL

39. Raman microprobe texture maps for samples in Fig. 38 and graph showing $\mathrm{J}_{\mathrm{c}}$ vs. YBCO thickness along with values for other ex situ films on various substrates.

40. Raman spectra from YBCO deposited by PLD on roll-textured $\mathrm{Ag}-\mathrm{Cu}$ substrates with varying $\mathrm{Cu}$ content along with plot of magnetization-based driving current, $\mathrm{I}_{500} / \mathrm{I}_{340}$ value, and $\mathrm{T}_{c}$ value for each film vs. $\mathrm{Cu}$ content of substrate

41. Raman spectra from YBCO deposited by PLD on Ag-0.2 at.\% $\mathrm{Cu}$ substrates at differing laser pulse energy densities along with plot of magnetization-based driving current and $\mathrm{I}_{500} / \mathrm{I}_{340}$ value vs. laser pulse energy density

42. Raman microprobe measurements from YBCO film vs. objective magnification, numerical aperture (NA), and working distance

43. Atom motion vectors for five Raman-active c-axis phonons of orthorhombic and tetragonal $\mathrm{MBCO}$ and typical frequency values for each crystal type

44. Raman spectra of bulk, melt-processed $\mathrm{MBCO} / \mathrm{O}$ and $\mathrm{MBCO} / \mathrm{T}$ samples for two orientations of incident excitation laser and observation direction.

45. Picture of reel-to-reel device used to feed and control longlength coated conductor specimens during examination by Raman microscopy

46. Time and temperature profiles used to produce time-gradientprocessed YBCO film on 1.25-m-long metal substrate 
47. Raman spectra of $\mathrm{Y}-\mathrm{BaF}_{2}-\mathrm{Cu}$ precursor at five increments in ramp zone of $1.25-\mathrm{m}$-long time-gradient-processed tape

48. Raman spectra recorded at $650^{\circ} \mathrm{C}$ ramp increment and at increment where precursor just reached transformation temperature $\left(740^{\circ} \mathrm{C}\right)$ before quench.

49. Raman spectra of increments along $1.25-\mathrm{m}$ time-gradientprocessed tape that reached $740^{\circ} \mathrm{C}$ along with their time at $740^{\circ} \mathrm{C}$

50. Plot correlating Raman bands for pure $\mathrm{Y}_{2} \mathrm{Cu}_{2} \mathrm{O}_{5}$ powder with bands seen in early stages of YBCO precursor transformation during time-gradient-processing of 1.25-m-long YBCO tape

51. Raman spectra from mid-portion of phase transition region, showing $\mathrm{Y}_{2} \mathrm{Cu}_{2} \mathrm{O}_{5}$ and $\mathrm{CuO}$ in early stages of $\mathrm{YBCO}$ formation along time-gradient-processed tape

52. Raman spectra from end-of-transformation region to beginning of over-processed region along 1.25-m YBCO time-gradientprocessed tape

53. Intensity-scale-expanded Raman spectra at four increments in "sweet spot" of 1.25-m-long YBCO time-gradient-processed tape

54. Three-dimensional plot of Raman spectral frequency and relative intensity versus position along the ORNL timegradient-processed tape

55. Top view of the Raman frequency-reaction time plane for the $\approx 300$-nm-thick ORNL time-gradient-processed tape during the ramp up and early soak period at $740^{\circ} \mathrm{C}$

56. Top view of the Raman frequency-reaction time plane for the $\approx 300$-nm-thick ORNL time-gradient-processed tape from early to late in the soak period at $740^{\circ} \mathrm{C}$

57. Top view of the Raman frequency-reaction time plane for the $\approx 1000 \mathrm{~nm}$-thick ORNL time-gradient-processed tape late in the soak period at $740^{\circ} \mathrm{C}$

58. $\mathrm{J}_{\mathrm{C}}$ of grain boundaries vs. grain boundary angle showing a crossover from film-limited $\mathrm{J}_{\mathrm{C}}$ to grain boundary-limited $\mathrm{J}_{\mathrm{C}}$ near $5^{\circ}$

59. Persistent current vs. temperature for $\mathrm{YBCO}$ ring with a $10^{\circ}$ grain boundary.

60. $\mathrm{J}_{\mathrm{c}}(\mathrm{T})$ for four $\mathrm{YBCO}$ rings, each with a $10^{\circ}$ grain boundary 56

61. $\mathrm{J}_{\mathrm{c}}(77 \mathrm{~K})$ vs. $\mathrm{T}_{\mathrm{c}}$ for the four $\mathrm{YBCO}$ rings with $10^{\circ}$ grain boundaries shown in Fig. 60 
62. $\mathrm{J}_{\mathrm{C}}(\mathrm{T})$ for $24^{\circ} \mathrm{YBCO}$ grain boundary in as-made state and after secondary oxygenation at $450^{\circ} \mathrm{C}$ and $400^{\circ} \mathrm{C}$.

63. $\mathrm{J}_{\mathrm{C}}(\mathrm{T})$ for $15^{\circ} \mathrm{YBCO}$ grain boundary in as-made state and after secondary oxygenation at $410^{\circ} \mathrm{C}, 380^{\circ} \mathrm{C}$ and $350^{\circ} \mathrm{C}$

64. $\mathrm{J}_{\mathrm{c}}(\mathrm{T})$ for $10^{\circ} \mathrm{YBCO}$ grain boundary in as-made state and after secondary oxygenation at $410^{\circ} \mathrm{C}, 380^{\circ} \mathrm{C}$ and $350^{\circ} \mathrm{C}$

65. Critical current ratio $\left(\mathrm{I}_{\mathrm{c}} / \mathrm{I}_{\mathrm{c} 0}\right)$ vs. applied bend strain $(\varepsilon)$ indicating critical strain $\varepsilon_{\mathrm{cr}}=0.17 \%$

66. Measured critical strain $\left(\varepsilon_{\mathrm{cr}}\right)$ vs. YBCO layer thickness.

67. Schematic comparison of standard AMSC HT-1 and five-step TSHT-type HT- 1 heat treatments for Bi-2223 conductors

\section{Tables}

1. X-ray diffraction full-width-at-half-maximum values showing the inplane texture of YBCO on SRO-buffered ISD-MgO substrate.

2. Summary of processing and performance results for $\mathrm{Ag} / \mathrm{Bi}-2223$ wires given standard AMSC first heat treatments (HT-1s) and Base Case (BC) Thermal Slide Heat Treatment (TSHT)-type HT-1s

3. Summary of processing and performance results for Ag/Bi-2223 wires given modified Thermal Slide Heat Treatment (TSHT)-type first heat treatments (HT-1s) 


\title{
PRACTICAL SUPERCONDUCTOR DEVELOPMENT FOR ELECTRICAL POWER APPLICATIONS
}

\author{
ANNUAL REPORT FOR FY 2003
}

\begin{abstract}
Most large-scale applications of high-critical-temperature superconductors will require conductors that can carry large currents in the presence of applied magnetic fields. This report describes progress at Argonne National Laboratory (ANL) in the research and development of practical superconducting components and devices. These efforts primarily focus on the use of $\mathrm{Y}-\mathrm{Ba}-\mathrm{Cu}-\mathrm{O}$ system in second-generation conductors, but they also include investigations of $\mathrm{Bi}-\mathrm{Pb}-\mathrm{Sr}-\mathrm{Ca}-\mathrm{Cu}-\mathrm{O}$ systems for use in first-generation conductors. Results are presented in the areas of processing first-generation superconductors and second-generation $(2 \mathrm{G})$ superconductors with several different architectures, applying Raman microscopy to the characterization of $2 \mathrm{G}$ conductors, studying the role of oxygen doping in the grain boundary transport of $2 \mathrm{G}$ conductors, and evaluating the mechanical properties of $2 \mathrm{G}$ conductors.
\end{abstract}

\section{Introduction}

The superconductor program at Argonne National Laboratory (ANL) is working to establish the technology that will enable broad-scale commercialization of high- $\mathrm{T}_{\mathcal{C}}$ superconductors (HTSs) in a wide variety of electric power applications. The principal objective of this program is to develop methods to fabricate and use structurally reliable HTSs for generating, transmitting, and storing electrical energy. This multifaceted effort seeks to improve the materials properties of HTSs through the understanding and manipulation of processing methods, develop practical methods for massproducing commercial conductors, fabricate and test prototype conductors, and study basic phenomena in order to understand and predict the performance of HTSs. In this program, the technology and science of fabricating long-length conductors is emphasized. Cooperative relationships with industrial and academic partners are integral to this program. At present, the program focuses primarily on $\mathrm{YBa}_{2} \mathrm{Cu}_{3} \mathrm{O}_{\mathrm{x}}(\mathrm{YBCO})$, but continues to explore materials in the bismuth-lead-strontium-calcium-copper oxide superconductor system.

Composite and monolithic conductors, in the form of wires, tapes, films, or other shapes, must satisfy several requirements. For most applications, the conductors must able to carry large currents in the presence of large magnetic fields and must be strong, flexible, and chemically and cryogenically stable. 
Potential applications for such conductors include transmission lines, motors, generators, transformers, magnetic energy storage devices, and electronics. Primary obstacles to the use of bulk HTSs include low critical current $\left(\mathrm{I}_{\mathrm{c}}\right)$ and critical current density $\left(\mathrm{J}_{\mathcal{C}}\right)$ in large applied magnetic fields, relatively poor mechanical properties, and difficulty in efficiently fabricating very long lengths with uniform properties. The ANL HTS program focuses on improving the methods for processing HTSs in order to eliminate each of these obstacles, with most of the effort centered on so-called second-generation YBCO-coated conductors.

This report reviews the technical progress in (1) fabricating YBCO-coated conductors with a variety of architectures, primarily using the Inclined Substrate Deposition (ISD) process; (2) utilizing Raman microscopy to characterize the microstructures of YBCO-coated conductors; (3) studying the influence of oxygen doping on the grain-boundary properties of YBCO-coated conductors; (4) evaluating the mechanical properties of $\mathrm{YBCO}$-coated conductors; and (5) optimizing the heat treatment of silver-sheathed $(\mathrm{Bi}, \mathrm{Pb})_{2} \mathrm{Sr}_{2} \mathrm{Ca}_{2} \mathrm{Cu}_{3} \mathrm{O}_{\mathrm{x}}(\mathrm{Bi}-2223)$ conductors.

\section{Technical Progress in 2002-2003}

\subsection{YBCO-Coated Conductors}

\subsubsection{Conductors with Standard ISD MgO Architecture}

ISD is a promising approach for fabricating biaxially textured template layers on non-textured metallic substrates. It requires neither an assisting ion source to develop biaxial alignment nor complicated high-temperature annealing treatments of the substrate. Because it yields the textured template films at room temperature, ISD is robust and relatively insensitive to heat treatment conditions. Using ISD, biaxially textured template layers have been made from both magnesium oxide (MgO) and yttria-stabilized zirconia (YSZ). In FY 2003, the focus at ANL has been on using template layers made by ISD of $\mathrm{MgO}$ to fabricate YBCO-coated conductors.

\subsection{1.a ISD MgO Process}

ISD MgO template layers are fabricated on polished Hastelloy C276 (HC) tapes using a procedure that is described elsewhere [1]. ISD MgO films contain grains that are columnar, stand nearly perpendicular to the substrate surface, and are terminated by $\mathrm{MgO}$ (002) planes. Because the [002] planes are tilted with respect to the substrate normal, ISD $\mathrm{MgO}$ films exhibit a roof-tile structure with a

relatively large surface roughness $(\approx 28 \mathrm{~nm})$. Deposition of an additional thin $(\approx 0.25-0.5 \mu \mathrm{m})$ homoepitaxial (HE) $\mathrm{MgO}$ layer at a substrate inclination, $\alpha$, of $0^{\circ}$ 
reduces the surface roughness to $\approx 9 \mathrm{~nm}$ and improves the biaxial texture, giving full width at half maximums (FWHMs) of 9.2 and $5.4^{\circ}$ for the $\mathrm{MgO}(002) \phi$ - and $\omega$-scans, respectively. In the "standard" ISD architecture, epitaxial layers of yttria-stabilized zirconia (YSZ) and ceria $\left(\mathrm{CeO}_{2}\right)$ are deposited on the $\mathrm{MgO}$ layer by pulsed laser deposition (PLD) before YBCO is deposited, because the lattice mismatch between $\mathrm{YBCO}$ and $\mathrm{MgO}$ is large $(\approx 9 \%)$. The standard ISD architecture is thus represented as $\mathrm{YBCO} / \mathrm{CeO}_{2} / \mathrm{YSZ} / \mathrm{HE}-\mathrm{MgO} / \mathrm{ISD}-\mathrm{MgO} / \mathrm{HC}$.

HC coupons $(\approx 0.1 \mathrm{~mm} x \approx 5 \mathrm{~mm} \times 1 \mathrm{~cm})$ are mechanically polished with diamond paste $(0.25 \mu \mathrm{m})$ before they are used as substrates for deposition of the standard ISD architecture. The polished substrates are mounted on a sample stage that can be tilted above the e-beam evaporator to give a range of substrate inclinations, $\alpha$, the angle between the substrate normal and the evaporation direction. For the studies described in this report, $\alpha$ was fixed at $55^{\circ}$. High purity oxygen is flowed into the system at $\approx 3 \mathrm{sccm}$ during film deposition. The base pressure is $1 \times 10^{-7}$ torr and rises to $\approx 2 \times 10^{-5}$ torr during deposition. Fused lumps of $\mathrm{MgO}$ (Alfa Aesar, $99.95 \%$ metals basis, 3-12 $\mathrm{mm}$ pieces) are used as the target material for growing $\mathrm{MgO}$ films. A quartz crystal monitor beside the sample stage monitors and controls the deposition rate at a fixed value in the range of $20-100 \AA / \mathrm{sec}$. The substrate temperature during deposition is maintained between room temperature and $50^{\circ} \mathrm{C}$. After the ISD film is deposited, a dense, thin, homoepitaxial layer of $\mathrm{MgO}$ is deposited at elevated temperature $\left(\approx 700^{\circ} \mathrm{C}\right)$ with $\alpha \approx 0^{\circ}$.

Buffer layers and YBCO films are deposited using a PLD system that is described elsewhere [2] and uses an optical beam raster to produce films with better uniformity over a broader area. Its laser beam is focused at the target through a quartz lens (1000 $\mathrm{mm}$ focal length) with an anti-reflective coating. A mirror that is part of the beam raster reflects the beam so that it hits the target at an incident angle of $45^{\circ}$. The rotating target carrousel carries four targets to accommodate the ablation of multiple layers without breaking vacuum. Commercial targets (99.999\% pure, $45 \mathrm{~mm}$ in diameter x $6 \mathrm{~mm}$ thick) from Superconductive Components are used. Substrates are attached to a heated sample stage with silver paste and heated to $700-800^{\circ} \mathrm{C}$ during deposition. The base pressure of the PLD chamber is $\approx 1 \times 10^{-5}$ torr. To maintain an operating pressure of 100-300 mtorr during deposition, the chamber is pumped with a molecular turbo pump while ultra-high-purity oxygen is flowed at $\approx 10 \mathrm{sccm}$. The spot size $\left(\approx 12 \mathrm{~mm}^{2}\right)$ of the laser beam on the rotating target gives an energy density of $\approx 2.0 \mathrm{~J} / \mathrm{cm}^{2}$. The distance between the target and the substrates is $\approx 7$ $\mathrm{cm}$. 
The superconducting critical transition temperature $\left(\mathrm{T}_{\mathrm{c}}\right)$ and critical current density $\left(\mathrm{J}_{\mathrm{C}}\right)$ are determined by an inductive method in which superconductor samples are placed between a primary and a secondary coil, each with an inner diameter of $\approx 1 \mathrm{~mm}$ and an outer diameter of $\approx 5 \mathrm{~mm}$. An alternating current of 1 $\mathrm{kHz}$ is introduced into the primary coil and detected by the secondary coil using a lock-in amplifier (Stanford Research Systems SR830 DSP). The transport $\mathrm{J}_{\mathrm{C}}$ of selected samples is measured by the four-point method at $77 \mathrm{~K}$ in liquid nitrogen using a $1 \mu \mathrm{V} / \mathrm{cm}$ criterion. Before measuring the transport $\mathrm{J}_{\mathrm{C}^{\prime}}$ the sample is first coated with silver (thickness $\approx 2 \mu \mathrm{m}$ ) by e-beam evaporation and then annealed at $400^{\circ} \mathrm{C}$ in flowing high-purity oxygen for $2 \mathrm{~h}$.

Film textures are characterized by $\mathrm{X}$-ray diffraction pole figure analysis using $\mathrm{Cu}-\mathrm{K}_{\alpha}$ radiation. The in-plane textures of the individual layers are characterized by the FWHMs of $\phi$-scans for the (002) reflections, and the out-ofplane textures are characterized by the FWHM of $\omega$-scans at the [001] pole for the same reflection. The in-plane texture of $\mathrm{YBCO}$ is measured using the FWHM of the YBCO (103) $\phi$-scan, and the out-of-plane texture is measured by the FWHM of the YBCO (005) $\omega$-scan. The surface morphology of individual layers is examined using a Hitachi S-4700-II scanning electron microscope (SEM). Selected area diffraction (SAD) is done using a Philips CM30 transmission electron microscope (TEM). Surface roughness is measured in the tapping mode using a Digital Instruments Dimension 3100 SPM Atomic Force Microscope (AFM).

\subsection{1.b Performance of Standard ISD Conductors}

By carefully controlling and optimizing the deposition parameters of individual layers during the ISD process, we sharpened considerably the biaxial texture of the YBCO layer during FY 2003, decreasing the full-width at half maximum (FWHM) of the YBCO (103) pole from $\approx 12^{\circ}$ on average to $\approx 10^{\circ}$. Along with the enhanced texture, YBCO films with the standard ISD architecture exhibited an increase in transport $\mathrm{J}_{\mathrm{C}}$. It is believed that the increase in $\mathrm{J}_{\mathrm{C}}$ resulted from the improvement in YBCO texture.

Figure 1 shows the results from transport measurements on a standard ISD sample (0.4- $\mu$ m-thick $\times 4$-mm-wide $\times 1$-cm-long) with a transport $\mathrm{J}_{\mathrm{C}}$ of $0.7 \mathrm{x}$ $10^{6} \mathrm{~A} / \mathrm{cm}^{2}$ at $77 \mathrm{~K}$ in self-field. Figure 2 shows the dependence of $\mathrm{J}_{\mathrm{c}}$ on applied magnetic field for the sample at $77 \mathrm{~K}$. Figure 3 shows the dependence of $\mathrm{J}_{\mathrm{c}}$ on $\xi$, the angle between the YBCO surface and the direction of an applied magnetic field of $1 \mathrm{~T}$. Sharp maxima in $\mathrm{J}_{\mathrm{c}}$ were observed when the field was aligned with the surface of the sample (Fig. 3), because the Lorenz force is perpendicular to the ab-plane in this configuration, and vortices induced by the applied magnetic field must cross the superconducting $\mathrm{CuO}_{2}$ planes in order for flux flow to occur. This 
observation is consistent with earlier reports that the c-axis of YBCO on c-axistilted ISD $\mathrm{MgO}$ is untilted, i.e., the $\mathrm{YBCO} \mathrm{c}$-axis is parallel to the substrate normal rather than the $\mathrm{c}$-axis of the $\mathrm{MgO}$ template layer.

Through an industrial collaboration with Universal Energy Systems (UES), ANL began in FY 2003 to explore the fabrication of long-length coated conductors by the ISD process. Using ISD technology developed at ANL, UES prepared 1-meter-long ISD MgO tapes on $\mathrm{HC}$, which were evaluated at ANL. The in-plane texture for these tapes was characterized by measuring the FWHM of the $\mathrm{MgO}$ (002) $\phi$-scan along the length of the tape. The results for one of these tapes are shown in Fig. 4 and indicate an average FWHM of $16.1^{\circ}$ with a standard deviation of $2.6^{\circ}$.

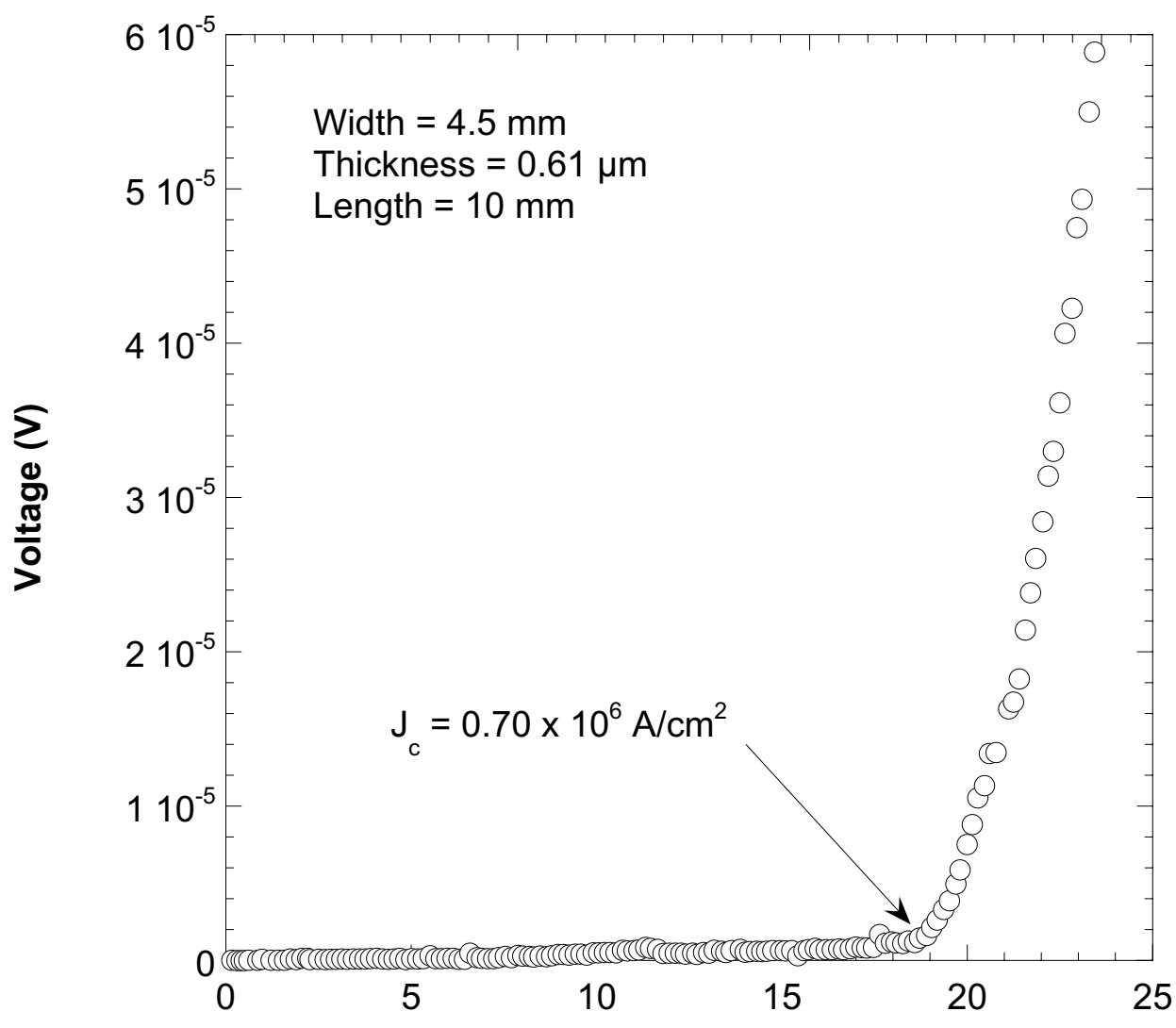

\section{Applied Current (A)}

Fig. 1 Transport measurements from sample with standard ISD architecture, $\mathrm{YBCO} / \mathrm{CeO}_{2} /$ yttria-stabilized zirconia (YSZ)/ISD $\mathrm{MgO} /$ Hastelloy C (HC). 


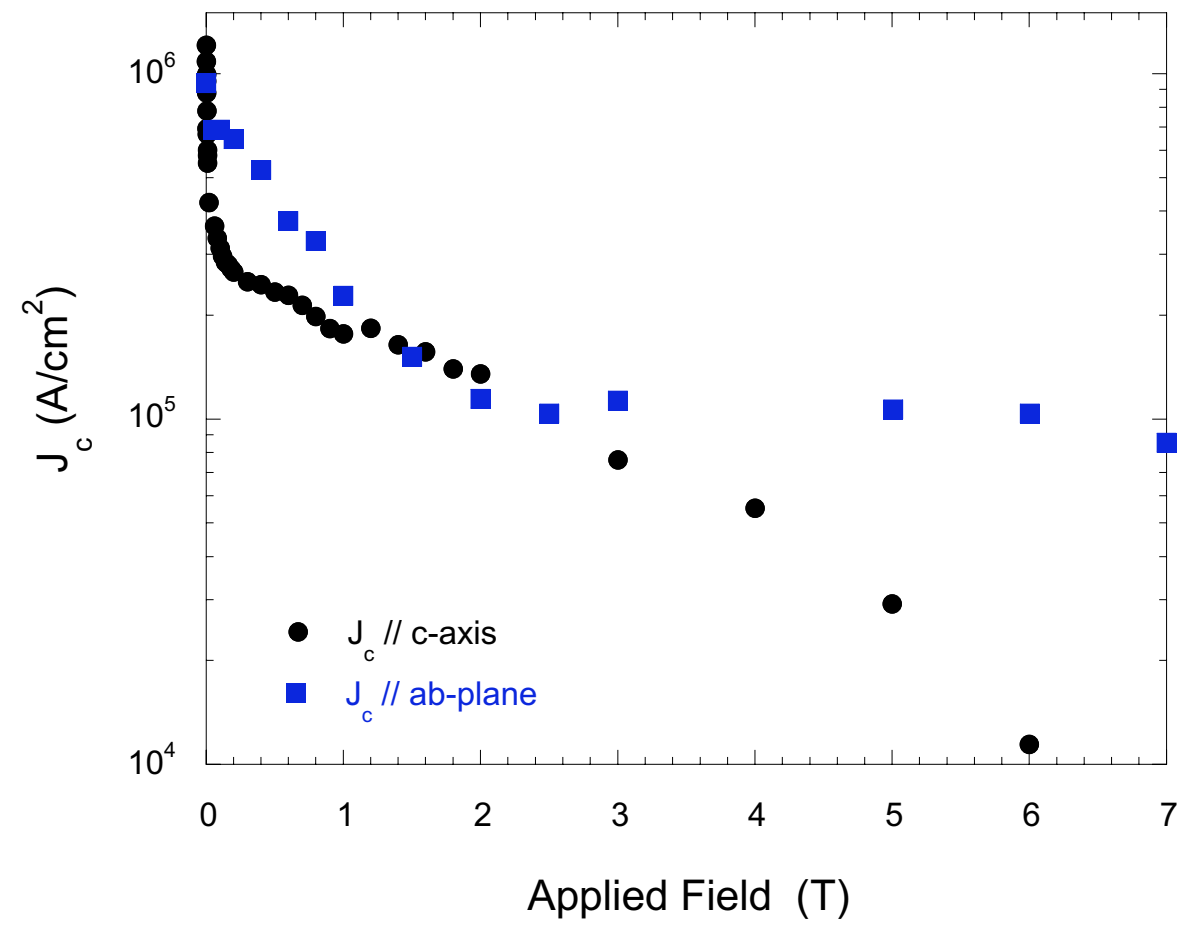

Fig. 2 Field-dependence of transport $J_{c}$ at $77 \mathrm{~K}$ for $Y B C O$ in "standard" ISD $\mathrm{MgO}$ architecture, $Y \mathrm{BCO} / \mathrm{CeO}_{2} / Y S Z / I S D$ $\mathrm{MgO} / \mathrm{HC}$.

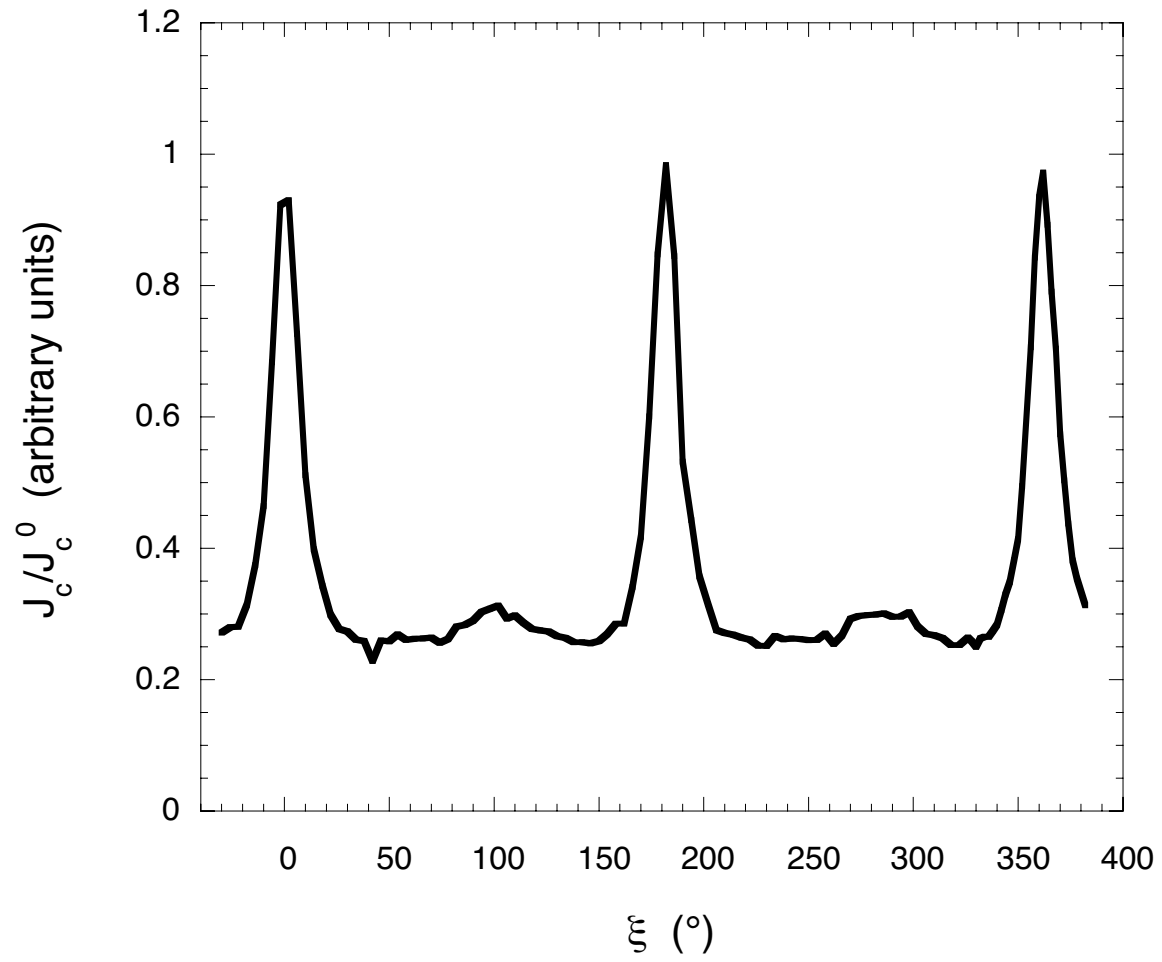

Fig. 3 Relative transport $J_{c}$ at $77 \mathrm{~K}$ in $1 \mathrm{~T}$ applied field as function of $\xi$, the angle between film surface and applied field, measured for YBCO in "standard" ISD MgO architecture. 


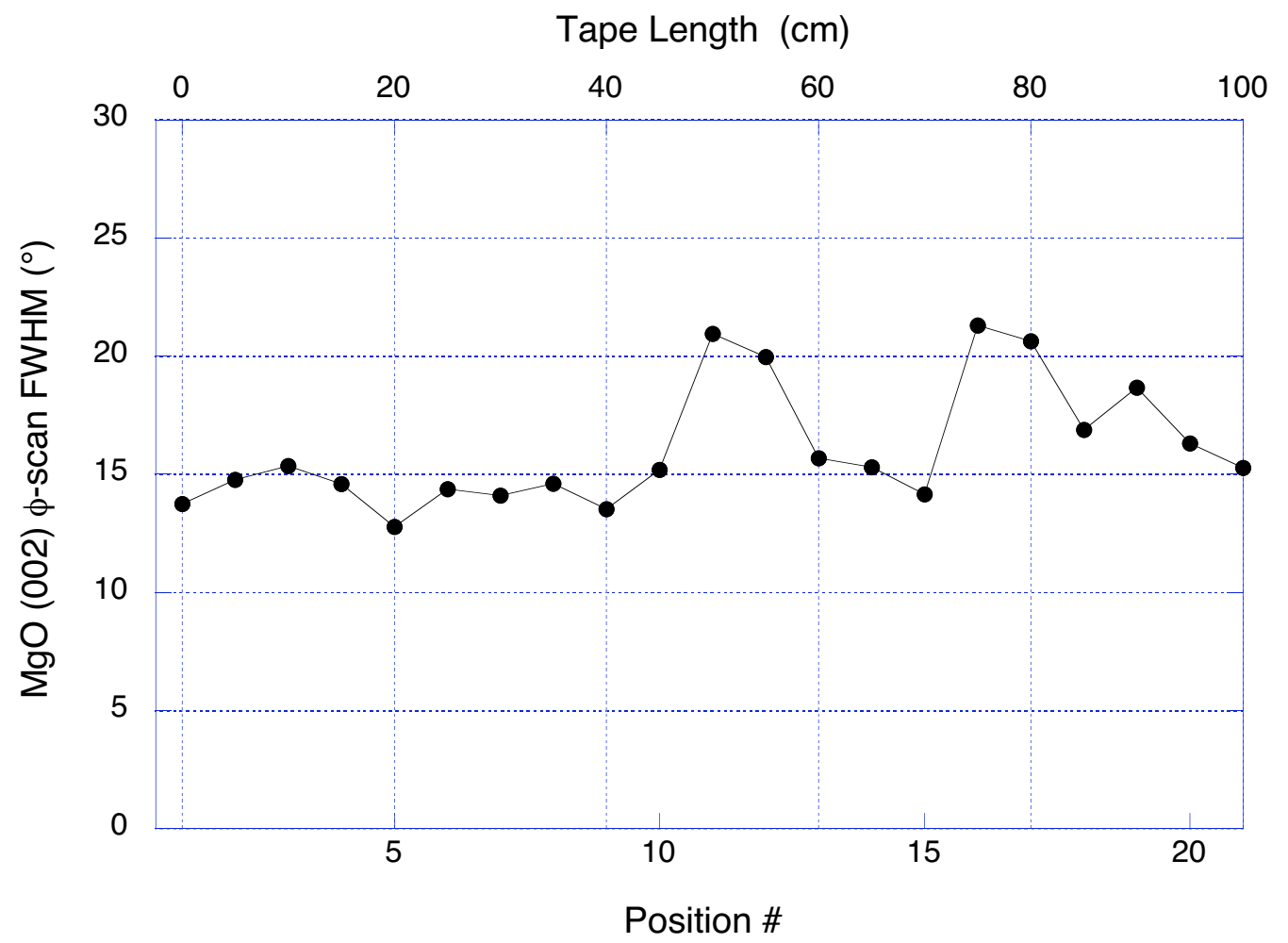

Fig. 4 Full width at half-maximum (FWHM) of $\mathrm{MgO}(002) \phi$-scans taken along the length of 1-m-long ISD MgO tape.

\subsection{1.c Texture Development/Crystallography of ISD MgO Conductors}

The Electron Microscopy Center at ANL conducted studies in FY 2003 to elucidate the texture formation mechanism during ISD, establish the microstructure of the textured template and subsequent layers, and identify the crystallography and orientation relationships between each layer.

Figure 5 shows a series of electron diffraction patterns from the ISD MgO template as a function of thickness. The broadening of the fundamental reflections is a measure of the range of grain orientations within the selected area. By measuring the angular width of the reflection, the texture can be determined as a function of thickness (equivalent to time), as shown in Fig. 6.

Two notable features are revealed in the plot of texture (as measured by the full-width of the electron diffraction reflection) as a function of thickness, seen in Fig. 6. First, biaxial texture develops very rapidly, reaching a plateau-like value at a thickness of $\approx 0.5 \mu \mathrm{m}$, which corresponds to a growth time of $\approx 2$ min under these deposition conditions. Second, deposition of the HE-MgO cap layer improves the biaxial texture, which has been confirmed by similar measurements on ISD films of differing thickness. For each ISD film thickness, the HE-MgO cap layer yields an improvement in texture. 


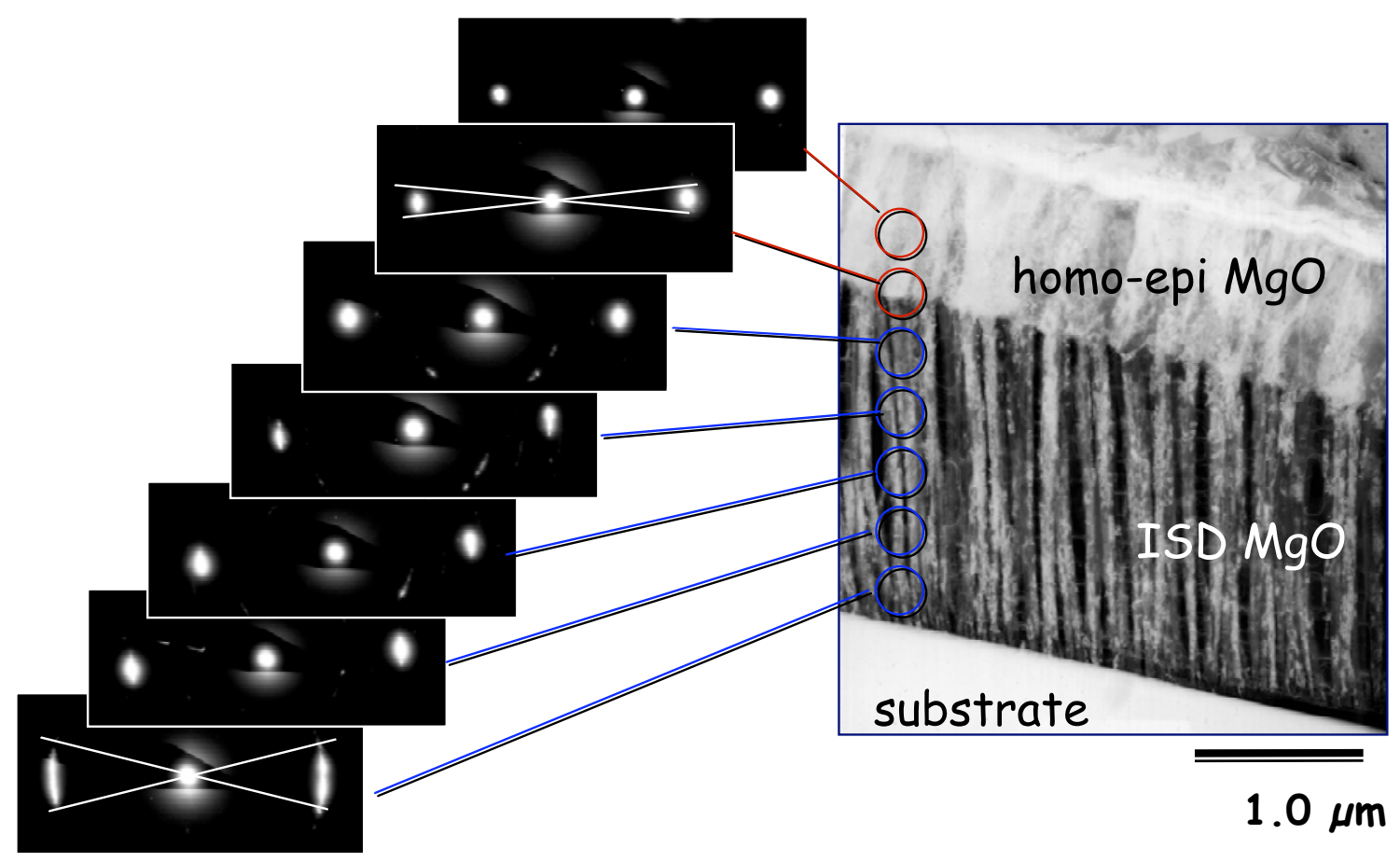

Fig. 5. Electron diffraction patterns from various points through the thickness of ISD-MgO template. Width of reflection indicates sharpness of texture.

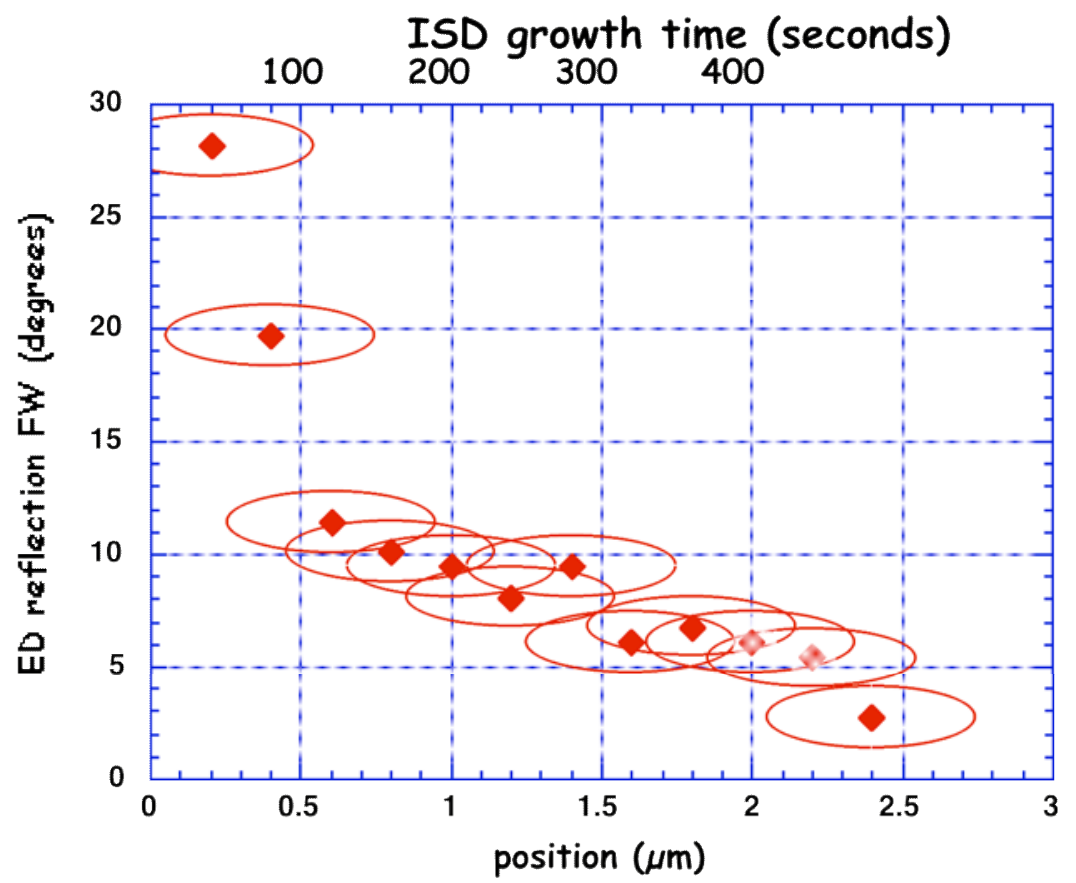

Fig. 6. Texture (as measured by full-width of electron diffraction reflection) through thickness of an ISD-MgO template. Lighter-shaded points (2.0-2.2 $\mu \mathrm{m})$ were measured from an area that included both ISD and $\mathrm{HE} \mathrm{MgO;}$ points to the left were measured from ISD MgO only, and point to the right from $\mathrm{HE} \mathrm{MgO}$ only. 
The rapid development of texture is significant in terms of the efficiency of the ISD process. The further evolution of texture during growth of the HE-MgO cap layer is shown in Fig. 7, where it is seen that a single grain in the HE layer caps three grains in the ISD layer. Considering the dimension out of the plane of the image, such HE grains appear to cap on the order of ten ISD grains. In a sort of averaging effect, the HE grains might improve the biaxial texture by adopting orientations in between those of the ISD grains beneath them.

Figure 8 shows a bright-field transmission electron microscopy (TEM) image and corresponding SAD pattern for the standard ISD architecture. In the image, the HE-MgO, YSZ, Ce-containing, and YBCO layers are each visible. The SAD pattern shows reflections from each of these layers, from which the orientation relationships can be established. In this particular case, the ceria has reacted during $\mathrm{YBCO}$ growth to form $\mathrm{BaCeO}_{3}$, so the orientation relationship before deposition cannot be determined. However, for each of the other layers, a specific orientation relationship is observed, as shown in Fig. 8. Thus, the epitaxial nature of growth through each of the layers is established.

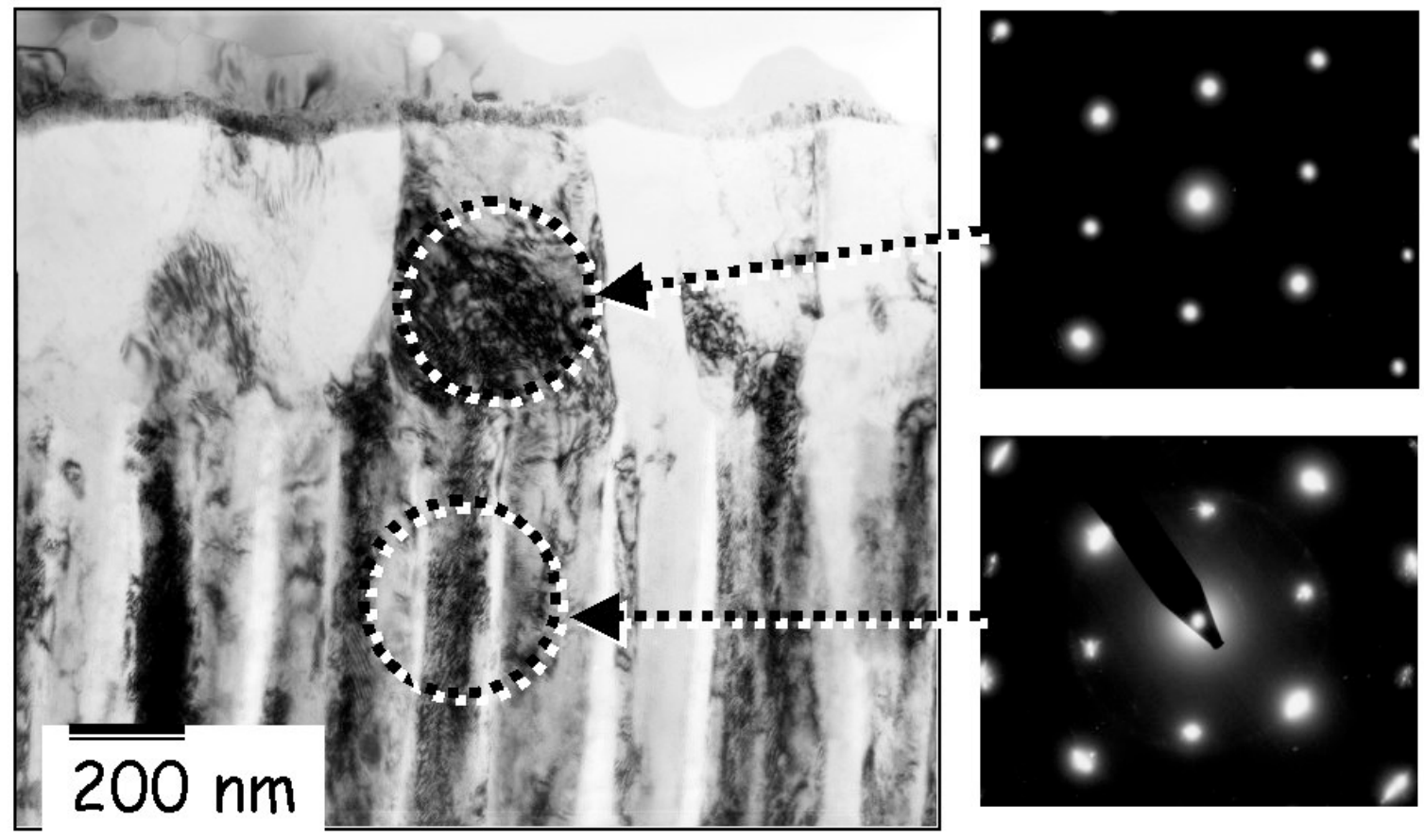

Fig. 7. Electron diffraction patterns from ISD-MgO and $\mathrm{HE}-\mathrm{MgO}$ layers showing enhancement of texture by HE layer. 


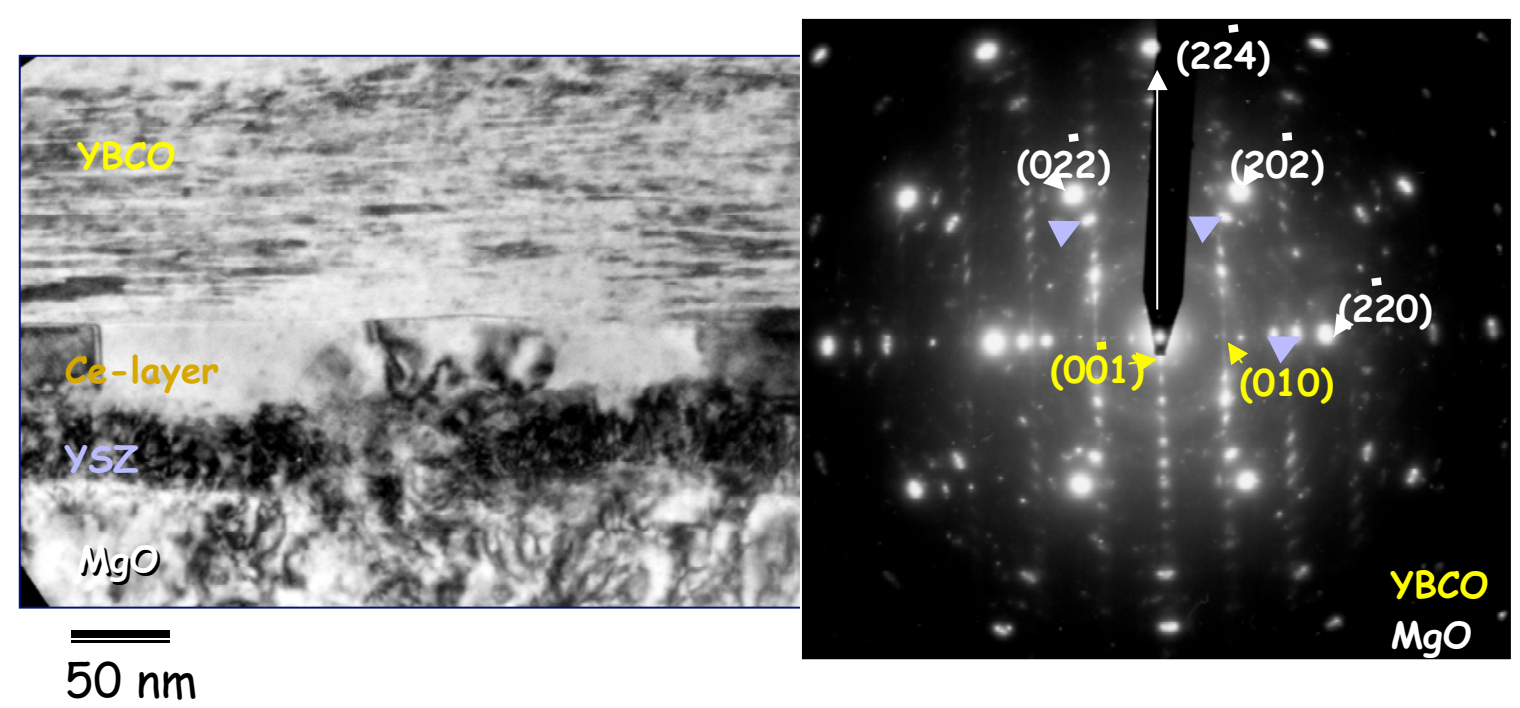

Fig. 8. Bright-field TEM image from ISD-based coated conductor and corresponding electron diffraction pattern used to determine orientation relationships.

An intriguing aspect of standard ISD conductors is that YBCO grows with its c-axis normal to the substrate (see Fig. 8), even though the $\mathrm{MgO}$ [002] planes are inclined with respect to the substrate normal. YBCO films with their c-axis oriented normal to the substrate offer several advantages. First, most design and modeling studies are based on films with this orientation, and second, films with c-axis-normal orientation can avoid the significant in-plane anisotropy that can result from inclined films.

The orientation of YBCO in ISD-based conductors depends on the crystallography of the underlying layers. For growth on some perovskite buffers, direct cube-on-cube epitaxy occurs, which leads to an inclined YBCO orientation. However the situation differs with the (211) surface plane that is presented by the "inclined" growth of the top $\mathrm{CeO}_{2}$ buffer layer. Because the lattice constants of $\mathrm{YBCO}$ and $\mathrm{CeO}_{2}$ differ considerably, cube-on-cube epitaxy in the inclined orientation leads to a relatively poor match for the YBCO (001) planes. In this situation, it is probable that crystalline anisotropy dictates YBCO growth in a c-axis orientation. Reciprocal space representations of the $\mathrm{CeO}_{2}$ [211] and YBCO [001] planes are shown in Fig. 9. From these representations, it is evident that the $\mathrm{CeO}_{2}$ (001)//YBCO (010) orientation relationship favors minimization of elastic interactions, and this is the orientation relationship that is observed experimentally. 


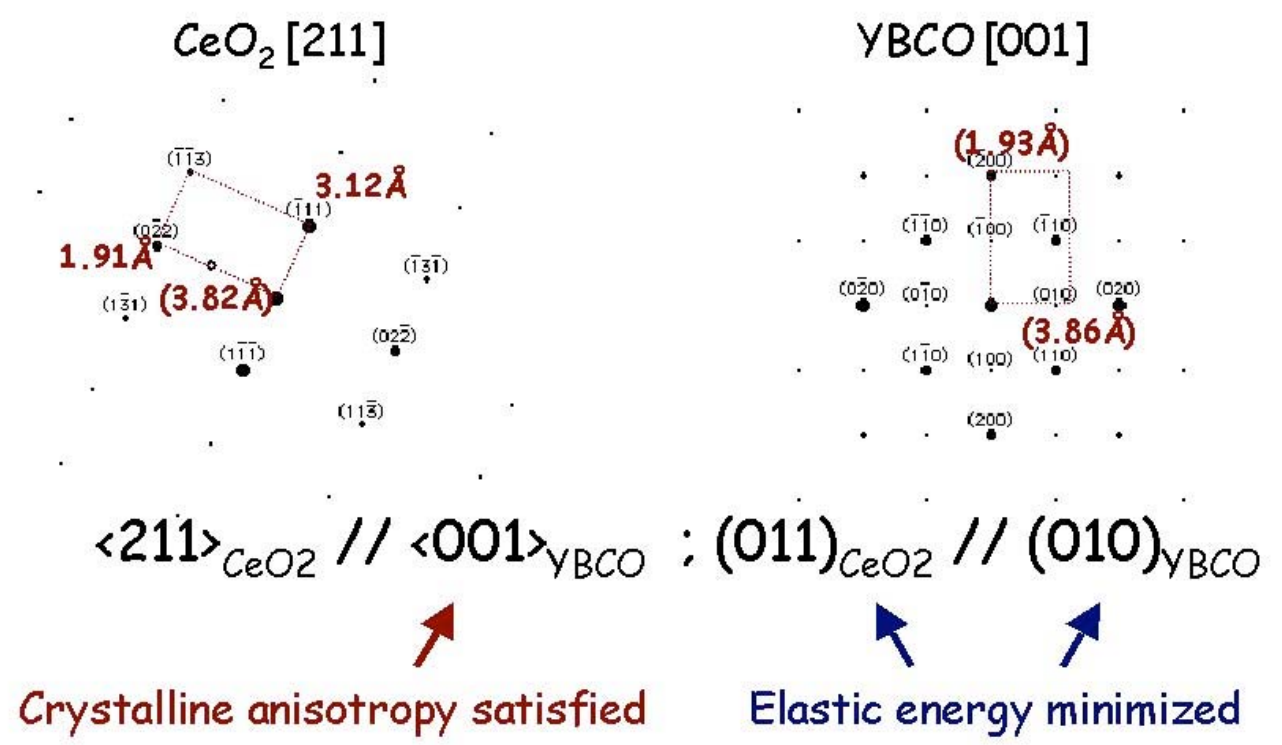

Fig. 9. Reciprocal space representations of $\mathrm{CeO}_{2}$ [211] and $\mathrm{YBCO}$ [001] planes showing that elastic interactions are minimized by orientation relationship $\mathrm{CeO}_{2}(001) / /$ YBCO (010).

\subsubsection{Conductors with $\mathrm{SrRuO}_{3}(\mathrm{SRO})$-buffered ISD $\mathrm{MgO}$ Architecture}

During FY 2003, we began investigating SRO as a potential alternative buffer layer for ISD coated conductors, i.e., YBCO-coated conductors made by the ISD method. SRO is chemically stable and exhibits good thermal and electrical conductivity. Its structure at room temperature is orthorhombic, with lattice parameters $\mathrm{a}=5.537 \AA, \mathrm{b}=7.852 \AA$, and $\mathrm{c}=5.573 \AA$ (JCPDS\# 89-5713). Because of its relatively small lattice mismatch $(\approx 6.5 \%)$ with $\mathrm{MgO}, \mathrm{SRO}$ can be grown epitaxially on the $\mathrm{MgO}$ template layer, and because its lattice mismatch with YBCO is intermediate between that of $\mathrm{YSZ}$ and $\mathrm{CeO}_{2}$, it might serve as a suitable alternative for one or both of the $\mathrm{YSZ}$ and $\mathrm{CeO}_{2}$ layers. The architecture of SRO-buffered conductors is thus: YBCO/SRO/ISD $\mathrm{MgO} / \mathrm{HC}$, where ISD $\mathrm{MgO}$ includes, in this case, both the ISD and $\mathrm{HE} \mathrm{MgO}$ layers.

\subsection{2.a Initial Study of Feasibility}

The efforts to develop SRO-buffered ISD conductors began by investigating YBCO films deposited on SRO-buffered $\mathrm{MgO}$ single crystals. Figures 10 and 11 show $\mathrm{T}_{\mathrm{C}}$ and $\mathrm{J}_{\mathrm{C}}$ curves for $\mathrm{a} \approx 0.3 \mu \mathrm{m}$ thick $\mathrm{YBCO}$ film on a single crystal $\mathrm{MgO}$ substrate. A sharp transition was measured with $\mathrm{T}_{\mathrm{C}}$ (onset) $=91 \mathrm{~K}$; the transport $\mathrm{J}_{\mathrm{C}}$ was $2.4 \times 10^{6} \mathrm{~A} / \mathrm{cm}^{2}$. These results showed that $\mathrm{SRO}$ is a good potential buffer layer for ISD conductors. 


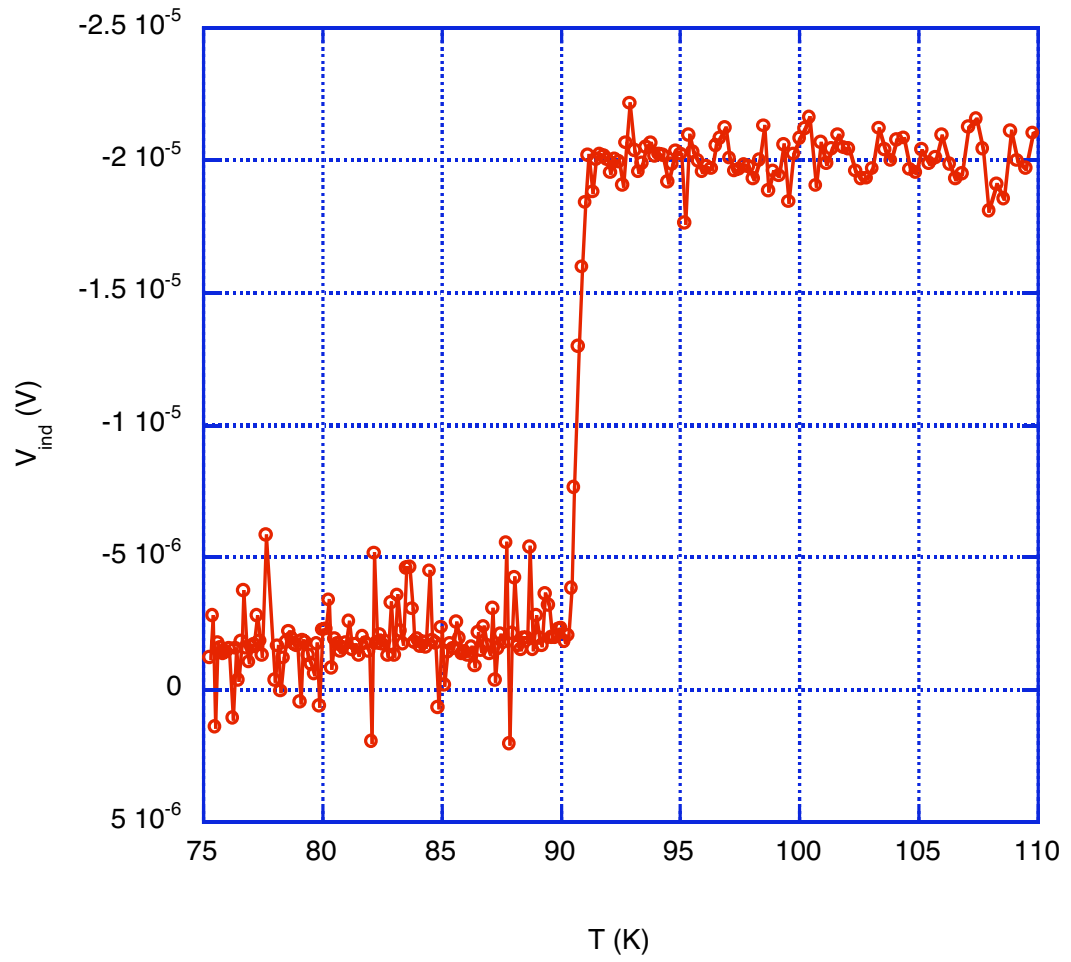

Fig. 10. $T_{c}$ transition curve for $a \approx 0.3 \mu \mathrm{m} Y B C O$ deposited on SRObuffered $\mathrm{MgO}$ single crystal.

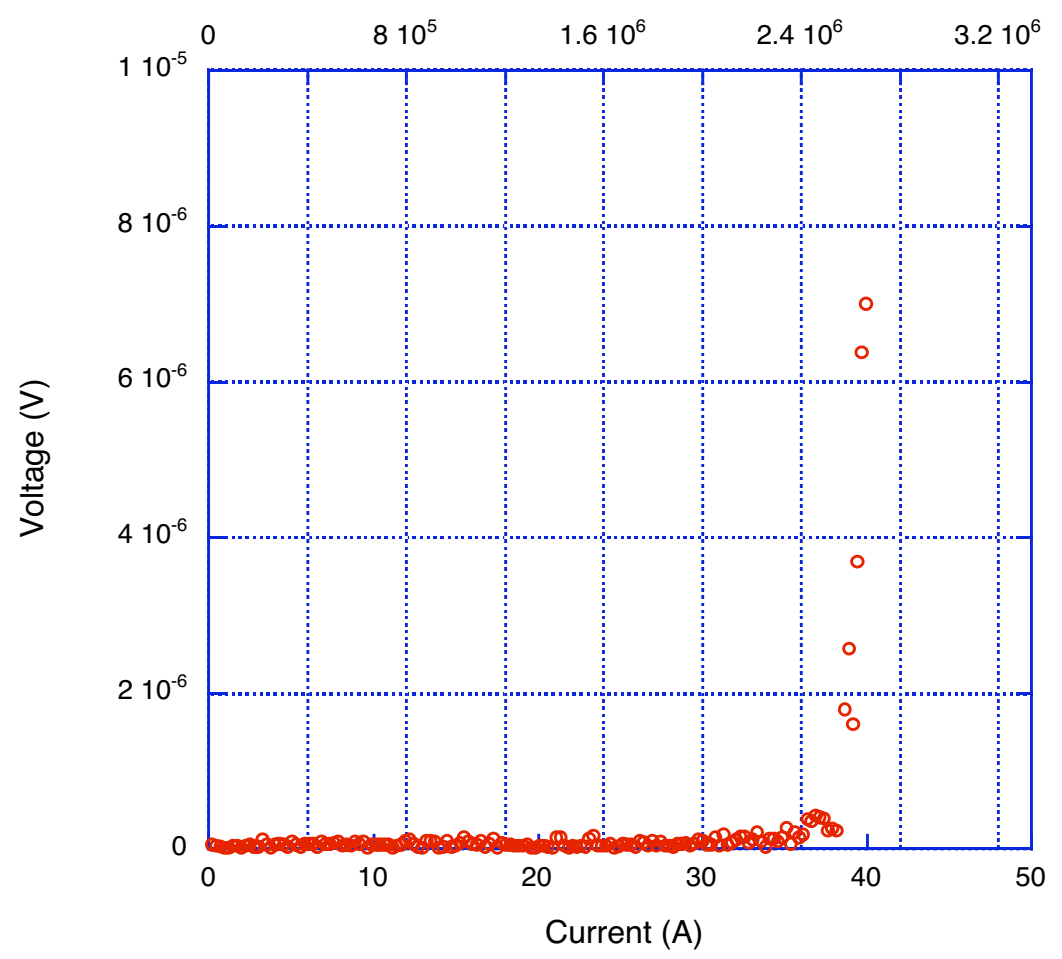

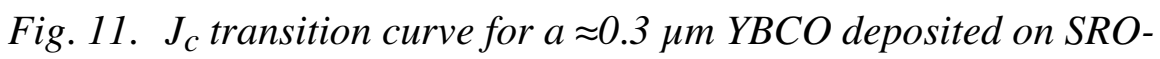
buffered $\mathrm{MgO}$ single crystal. 
To further test the suitability of SRO as a buffer layer, we fabricated several YBCO films on SRO-buffered ISD MgO templates. The ISD MgO template films were deposited on short length $\mathrm{HC}$ substrates by electron-beam evaporation using the process described above. The substrate with its ISD MgO layer was attached to a heated sample stage by silver paste. SRO films were deposited by PLD at $4 \mathrm{~Hz}$ at $760-780^{\circ} \mathrm{C}$ in an atmosphere of 50 mtorr of oxygen. The distance between the rotating SRO target and the ISD $\mathrm{MgO} / \mathrm{HC}$ substrate was $7 \mathrm{~cm}$. The base pressure of the PLD chamber was $1 \times 10^{-5}$ torr, and the desired oxygen pressure ( $50 \mathrm{mtorr}$ ) was achieved by flowing $\approx 10 \mathrm{sccm}$ high-purity oxygen through the system.

Plan-view images (Figs. 12a and 12b) show that SRO deposited on an ISD $\mathrm{MgO} / \mathrm{HC}$ substrate is dense and contains a few inclusions. The SRO grain size is small, but the grain size is larger in the YBCO film grown on the SRO buffer layer, as seen in Figs. 12c and 12d. It is also seen, however, that the YBCO grains are not well connected, indicating that the conditions for depositing $\mathrm{YBCO}$ on SRO needed further optimization at this point.

Figure 13 shows $\mathrm{X}$-ray diffraction pole figures for $\mathrm{YBCO}$ that was deposited on an SRO-buffered ISD MgO/HC substrate. Cube-on-cube epitaxial growth was observed. Good in-plane alignment was observed for the SRO buffer layer and the YBCO film, as indicated in Table 1. Unlike YBCO on ISD conductors with the standard architecture, however, the c-axis of $\mathrm{YBCO}$ is tilted with respect to the substrate in $\mathrm{YBCO}$ grown on the SRO buffer layer.

Table 1. In-plane texture of YBCO on SRO-buffered ISD-MgO substrate

\begin{tabular}{cc}
\hline Diffraction plane & FWHM $\left(^{\circ}\right)$ \\
\hline $\operatorname{MgO}(002)$ & 9.62 \\
SRO (020) & 6.48 \\
YBCO (005) & 6.39 \\
YBCO $(103)$ & 6.88 \\
\hline
\end{tabular}

A short-length YBCO sample (1.0 cm wide $x 1.2 \mathrm{~cm}$ long) was coated with silver (thickness $\approx 2 \mu \mathrm{m}$ ) and annealed in oxygen at $400^{\circ} \mathrm{C}$ for 60 minutes to measure its transport $\mathrm{J}_{\mathrm{c}}$. The resulting I-V curve is shown in Fig. 14. Considering the small dimensions of the sample, $\mathrm{J}_{\mathrm{C}}$ is essentially the same in both directions, i.e., parallel and perpendicular to the ridges formed in the ISD process. Within experimental uncertainty, no directional anisotropy was observed. However, the transition along the length direction was sharper than that along the width direction. $\mathrm{J}_{\mathrm{c}}$ measured at $77 \mathrm{~K}$ in self-field was $\approx 30 \mathrm{kA} / \mathrm{cm}^{2}$, even though the biaxial alignment in the film was reasonably good. A second sample of YBCO grown on SRO-buffered ISD $\mathrm{MgO} / \mathrm{HC}$ gave a transport $\mathrm{J}_{\mathrm{C}}$ of $100 \mathrm{kA} / \mathrm{cm}^{2}$. 

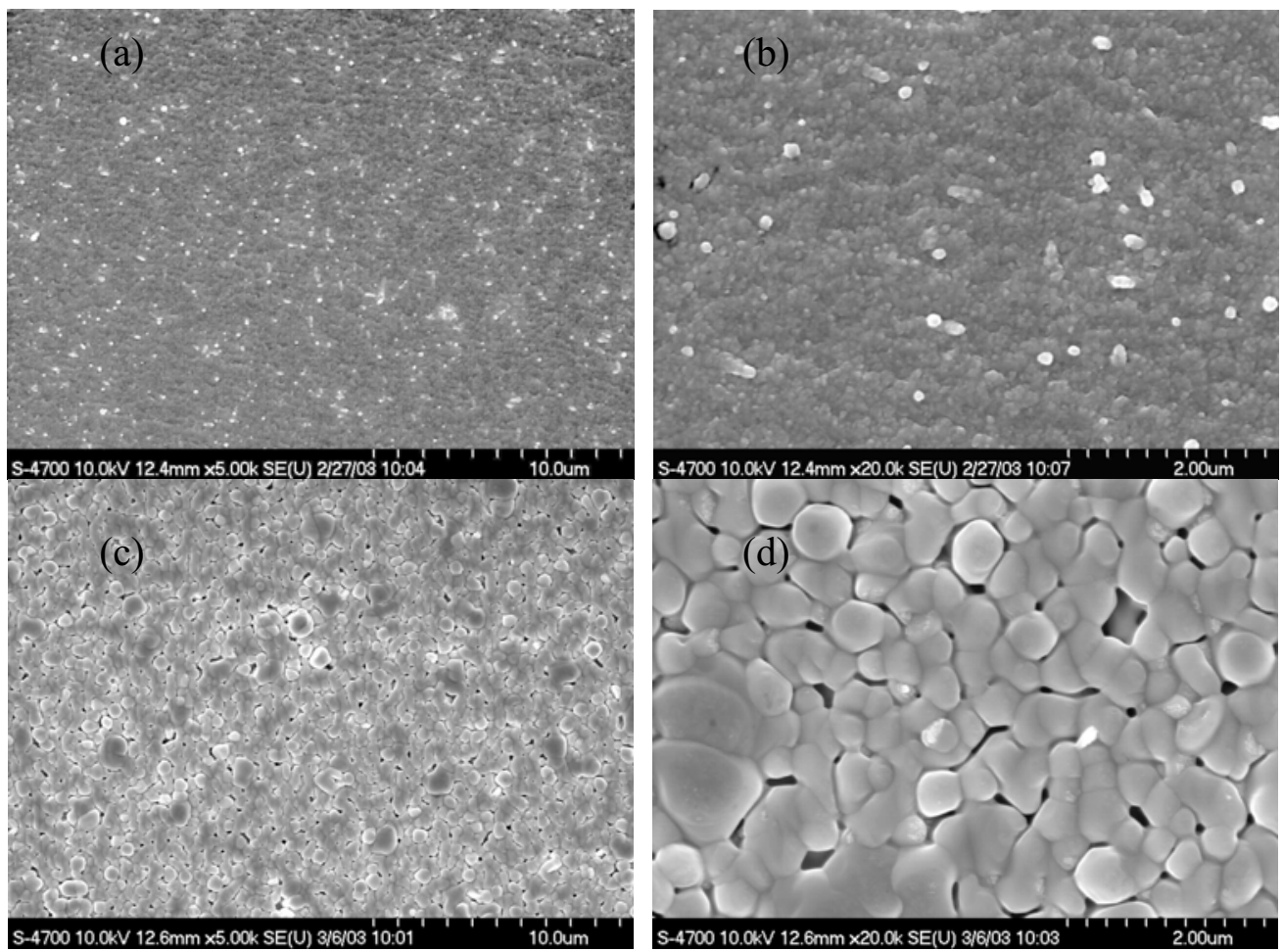

Fig. 12. Plan-view SEM images of (a) and (b) SRO buffer layer, and (c) and (d) YBCO film deposited on ISD MgO substrate.

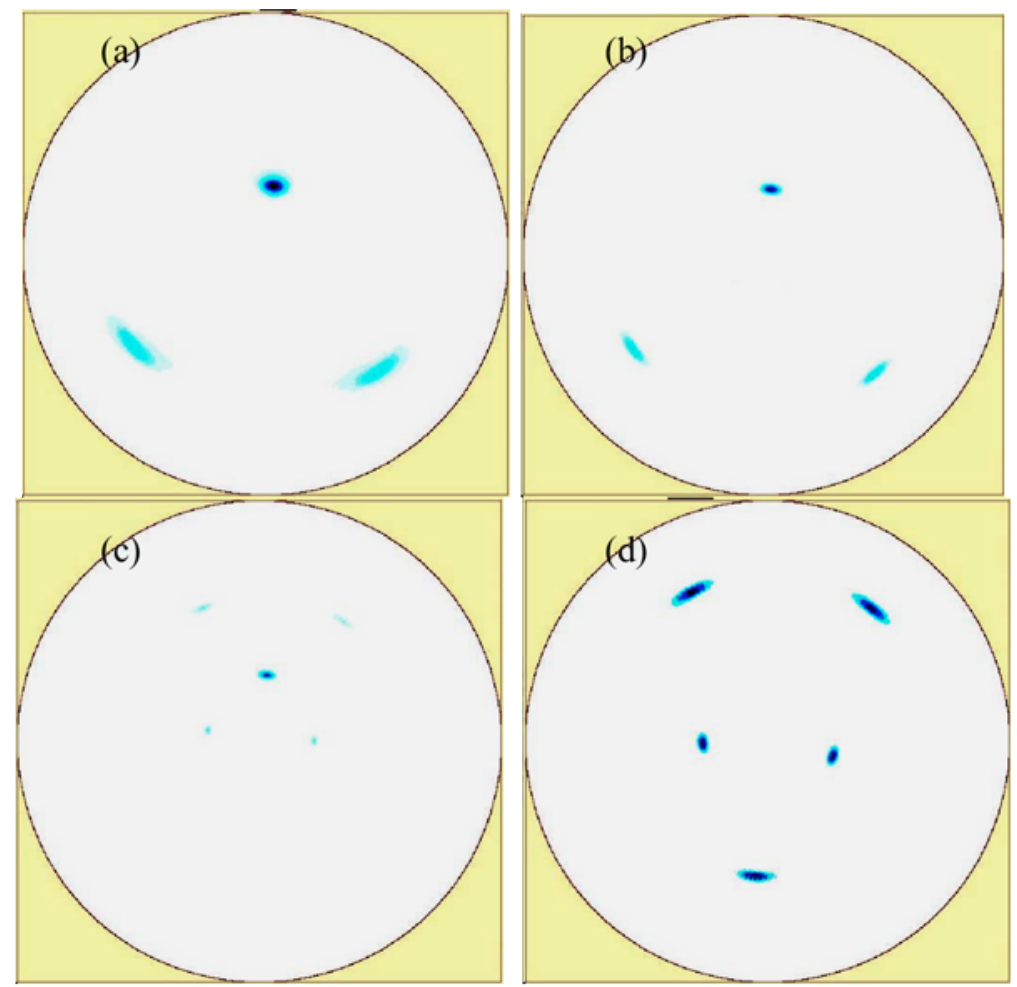

Fig. 13. X-ray pole figures: (a) MgO(002), (b) SRO(020), (c) $Y B C O(005)$, and (d) YBCO(103) measured on YBCO on SRO-buffered ISD-MgO substrate. 


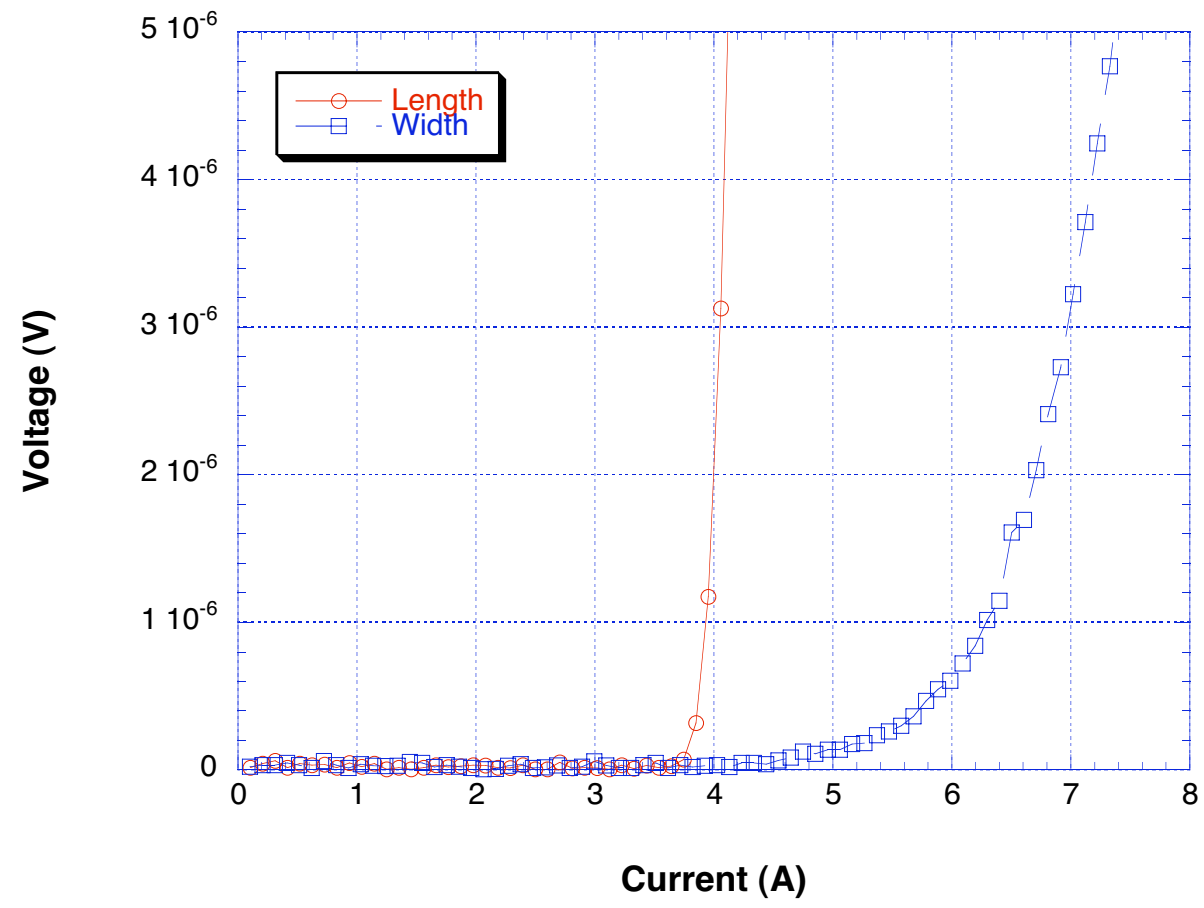

Fig. 14. Voltage vs. current for YBCO deposited on SRO-buffered ISD MgO/HC substrate.

\subsection{2.b Study of SRO-Buffered Single Crystal MgO Substrates}

Although the in-plane alignment for $\mathrm{YBCO}$ was significantly sharper on SRO-buffered ISD conductors than on standard ISD conductors, the initial SRObuffered conductors did not exhibit significantly better transport properties. Believing that improving the deposition of the SRO and YBCO layers would increase the transport $\mathrm{J}_{\mathrm{c}}$, we focused on optimizing the PLD of SRO on $\mathrm{MgO}$ single-crystal substrates and the subsequent deposition of $\mathrm{YBCO}$, primarily investigating the effect of deposition temperatures in the range $750-790^{\circ} \mathrm{C}$.

The SRO and YBCO films in this study were deposited on single crystal $\mathrm{MgO}$ (001) substrates using PLD. A KrF excimer laser (Lambda Physik, Compex 201) with a wavelength of $248 \mathrm{~nm}$ and pulse duration of $25 \mathrm{~ns}$ was focused onto a target rotating at $8 \mathrm{rpm}$. Both the YBCO and SRO targets (supplied by Praxair Surface Technology) were $2.54 \mathrm{~cm}$ in dia. and $0.63 \mathrm{~cm}$ thick. A laser beam with energy of $128 \mathrm{~mJ} /$ pulse and $4 \mathrm{~Hz}$ frequency was used to fabricate SRO films, and a laser beam with energy of $140 \mathrm{~mJ} /$ pulse and $8 \mathrm{~Hz}$ frequency was used for YBCO films. Prior to deposition of SRO, the $\mathrm{MgO}$ substrates were ultrasonically cleaned in acetone and then in methyl alcohol for $10 \mathrm{~min}$. They were then mounted on a substrate heater using silver paste. The distance between the substrate and target was $6.5 \mathrm{~cm}$. The $200 \mathrm{~nm}$ SRO films were deposited on $\mathrm{MgO}$ 
at 750,760,770,780, and $790^{\circ} \mathrm{C}$ in $50 \mathrm{mtorr} \mathrm{O}_{2}$. After deposition of the SRO film, it was cooled to room temperature in $\mathrm{O}_{2}$ at ambient pressure. For the growth of YBCO films, SRO buffer layers of thickness $100 \mathrm{~nm}$ were deposited at $770^{\circ} \mathrm{C}$ in 50 mtorr $\mathrm{O}_{2}$ on $\mathrm{MgO}$ single crystal substrates. YBCO films were subsequently deposited onto the SRO buffer layers at 750, 760, 770, 780, and $790^{\circ} \mathrm{C}$ in $240 \mathrm{mtorr}$ $\mathrm{O}_{2}$. The YBCO films were then annealed inside the chamber at $450^{\circ} \mathrm{C}$ in $1 \mathrm{~atm} \mathrm{O}_{2}$ for $90 \mathrm{~min}$. Film thicknesses were measured using a Dektak 3 profilometer (Veeco, Metrology group).

The $T_{C}$ of the YBCO films was measured using an inductive magnetization method in which the sample is placed between a primary and a secondary coil, and an alternating current is applied to the primary coil. A lock-in amplifier was used to measure the voltage that the penetrating field produced in the secondary coil. J $\mathrm{J}_{\mathrm{C}}$ 's were measured using a standard four-probe method at $\mathrm{T}=77 \mathrm{~K}$ after the YBCO films were coated with $2 \mu \mathrm{m}$ of silver using electron beam evaporation. The in-plane texture of the films was studied by X-ray $\phi$-scan, whereas the out-of-plane texture of the films was examined by X-ray $\omega$-scan. Scanning electron microscopy (SEM) was used to study the surface morphology of the films, and Raman microspectroscopy (Renishaw RM2000 Raman microscope) provided information regarding the chemistry of the films.

To investigate the effect of deposition temperature on the texture of the SRO buffer layer, SRO films were deposited on $\mathrm{MgO}$ single-crystal substrates at temperatures of $750,760,770,780$, and $790^{\circ} \mathrm{C}$. The (220) $\phi$-scan of SRO deposited at $770^{\circ} \mathrm{C}$ had a FWHM of $0.46^{\circ}$ (Fig. 15a), indicating high in-plane crystallinity. SRO films deposited at other temperatures also exhibited good in-plane texture (see Table 1), with FWHM for the (220) $\phi$-scan decreasing as the deposition temperature increased. The out-of-plane texture was not as sharp as the in-plane texture, with $\mathrm{FWHM}$ values for $\mathrm{SRO}(020) \omega$-scans ranging from $1.35-1.79^{\circ}$ (see Table 1). An SRO deposition temperature of $770^{\circ} \mathrm{C}$ gave the smallest FWHM for the (020) $\omega$-scan (Fig. 15b) and was used to prepare the SRO-buffered $\mathrm{MgO}$ substrates on which YBCO was deposited.

Figure 16 shows the FWHM values for $\mathrm{YBCO}(113) \phi$ - and $\mathrm{YBCO}(003) \omega$ scans for $\mathrm{YBCO}$ deposited at 750, 760, 770, 780, and $790^{\circ} \mathrm{C}$ on SRO-buffered MgO. The best in- and out-of-plane textures were obtained for the $\mathrm{YBCO}$ film deposited at $770^{\circ} \mathrm{C}$. The $\mathrm{YBCO}(113) \phi$ - and $\mathrm{YBCO}(003) \omega$-scans for $\mathrm{YBCO}$ deposited at $770^{\circ} \mathrm{C}$ are shown in Fig. 17 , and gave a FWHM of $\approx 1.39^{\circ}$ for the $\phi$ scan (Fig. 17a) and $\approx 0.7^{\circ}$ for the $\omega$-scan (Fig. 17b). 

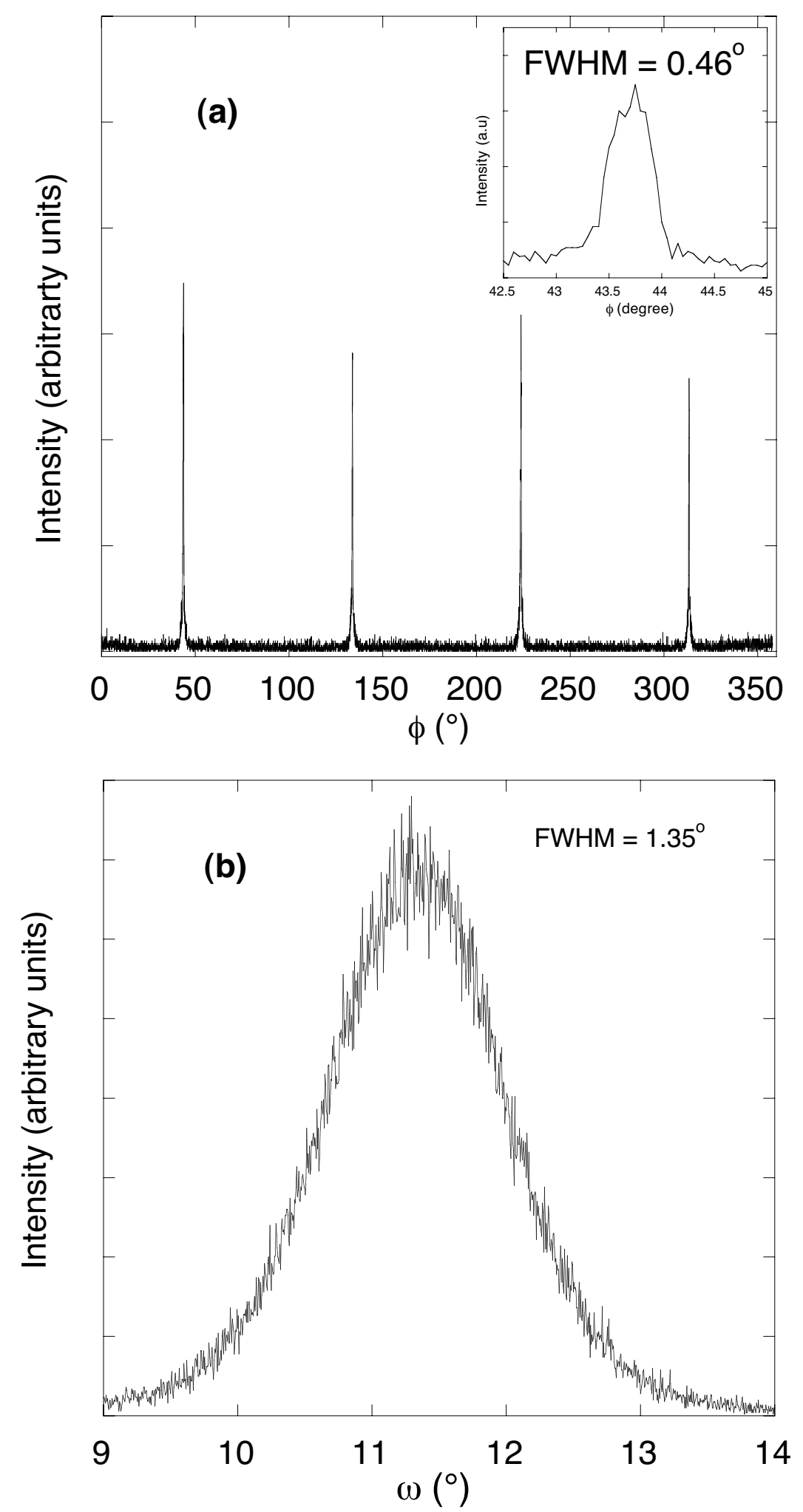

Fig. 15. X-ray scans showing in-plane and out-of-plane texture of SRO deposited by PLD at $770^{\circ} \mathrm{C}$ on $\mathrm{MgO}$ single crystal substrate. (a) $S R O(220) \phi$-scan, and (b) $S R O(020) \omega$-scan. 


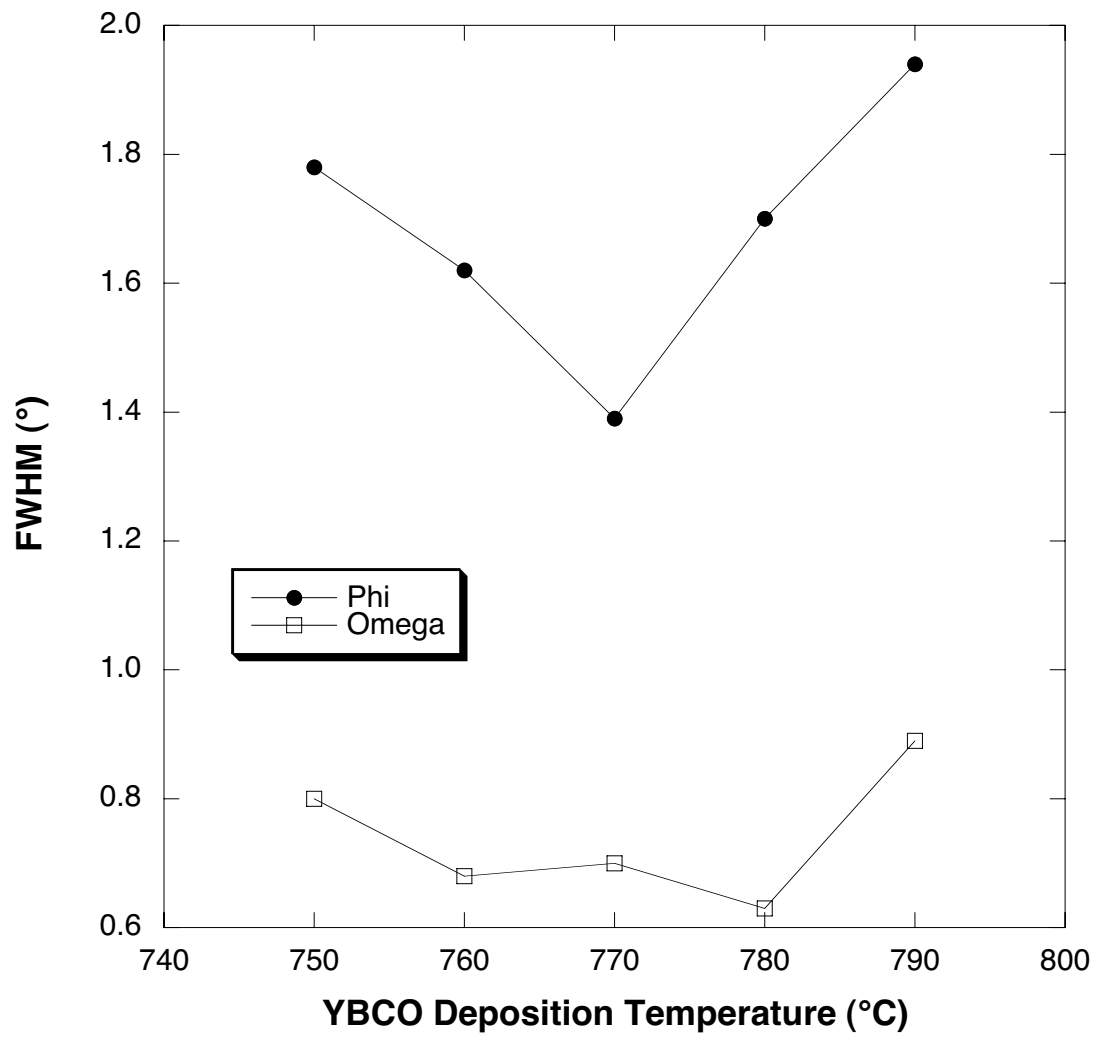

Fig. 16. Full width at half-maximum (FWHM) of YBCO(113) $\phi$-and (003) $\omega$-scans for YBCO deposited by PLD on SRO-buffered $\mathrm{MgO}$ single crystal substrates versus temperature of $Y B C O$ deposition.
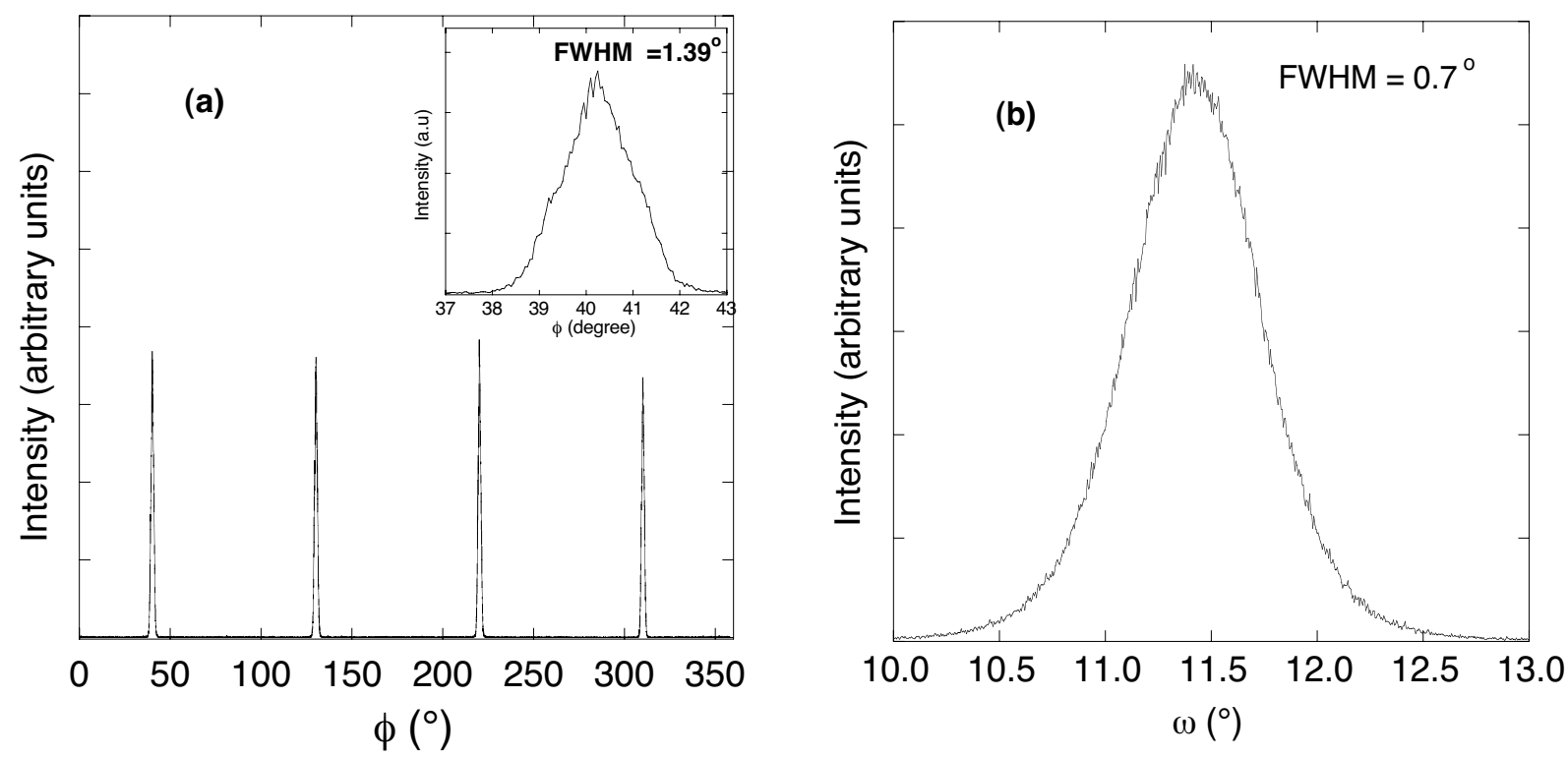

Fig. 17. X-ray scans showing in-plane and out-of-plane texture of $Y B C O$ deposited at $770^{\circ} \mathrm{C}$ on SRO-buffered $\mathrm{MgO}$ single crystal substrate. $S R O$ was deposited on $\mathrm{MgO}$ single crystal at $770^{\circ} \mathrm{C}$. (a) $Y B C O(113) \phi$-scan, (b) YBCO (003) $\omega$ - scan. 
The $\mathrm{T}_{\mathrm{C}}$ and transition width $(\Delta \mathrm{T})$ are plotted in Fig. 18 versus the $\mathrm{YBCO}$ deposition temperature for $\mathrm{YBCO}$ on SRO-buffered $\mathrm{MgO}$ single-crystal substrates. In all these samples, the SRO buffer layer was deposited at $770^{\circ} \mathrm{C}$. All films had $\mathrm{T}_{\mathrm{C}}>90 \mathrm{~K}$ with a transition width $<1 \mathrm{~K}$. Figure 19 shows the superconducting transition for $\mathrm{YBCO}$ deposited at $770^{\circ} \mathrm{C}$, with $\mathrm{T}_{\mathrm{C}}$ (onset) of $91 \mathrm{~K}$ and transition width $(\Delta \mathrm{T})$ of $0.6 \mathrm{~K}$. Figure 20 shows that the film deposited at $770^{\circ} \mathrm{C}$ had the highest transport $\mathrm{J}_{\mathrm{C}}, 2.50 \times 10^{6} \mathrm{~A} / \mathrm{cm}^{2}$, and that the $\mathrm{J}_{\mathrm{C}}$ decreased sharply for films deposited at higher temperatures. At $790^{\circ} \mathrm{C}$, the $\mathrm{J}_{\mathrm{C}}$ was $6 \times$ $10^{5} \mathrm{~A} / \mathrm{cm}^{2}$. While the $\mathrm{T}_{\mathrm{c}}$ and the $\Delta \mathrm{T}$ values do not depend strongly on the deposition temperature, the $\mathrm{J}_{\mathrm{C}}$ values show a maximum near $770^{\circ} \mathrm{C}$.

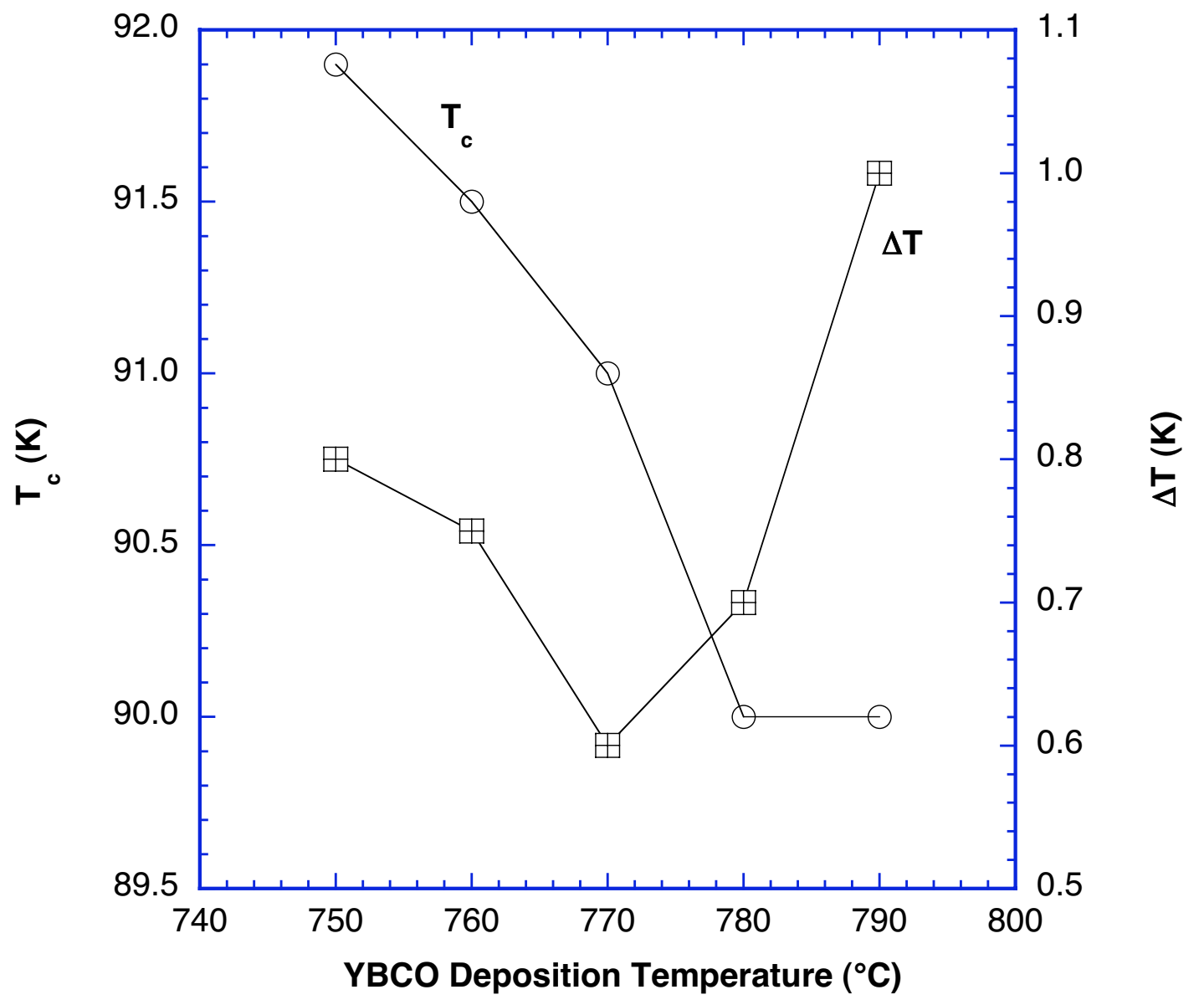

Fig. 18. Critical temperature, $T_{c}$, and superconducting transition width, $\Delta T$, for YBCO films deposited by PLD on SRO-buffered single crystal $\mathrm{MgO}$ substrates versus temperature of YBCO deposition. 


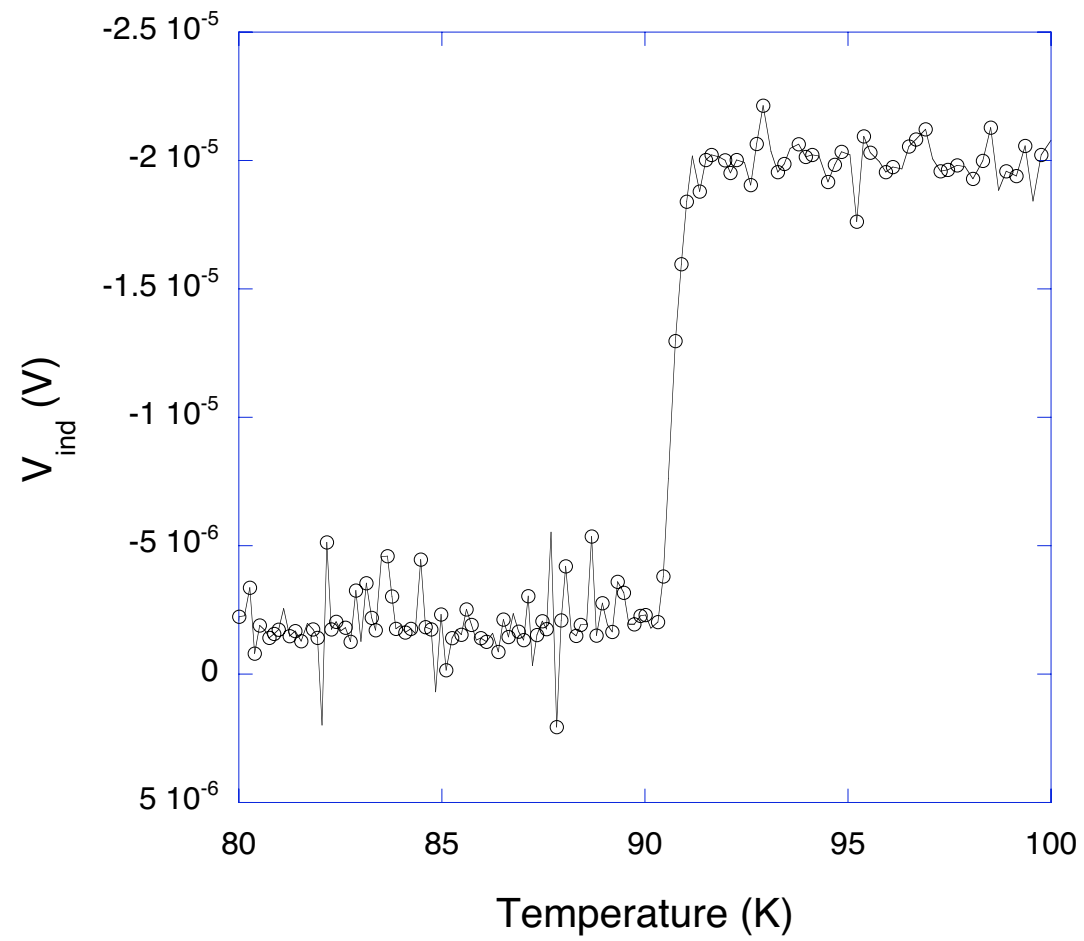

Fig. 19. Superconducting transition, measured inductively, for YBCO deposited at $770^{\circ} \mathrm{C}$ on SRO-buffered $\mathrm{MgO}$ single crystal substrate.

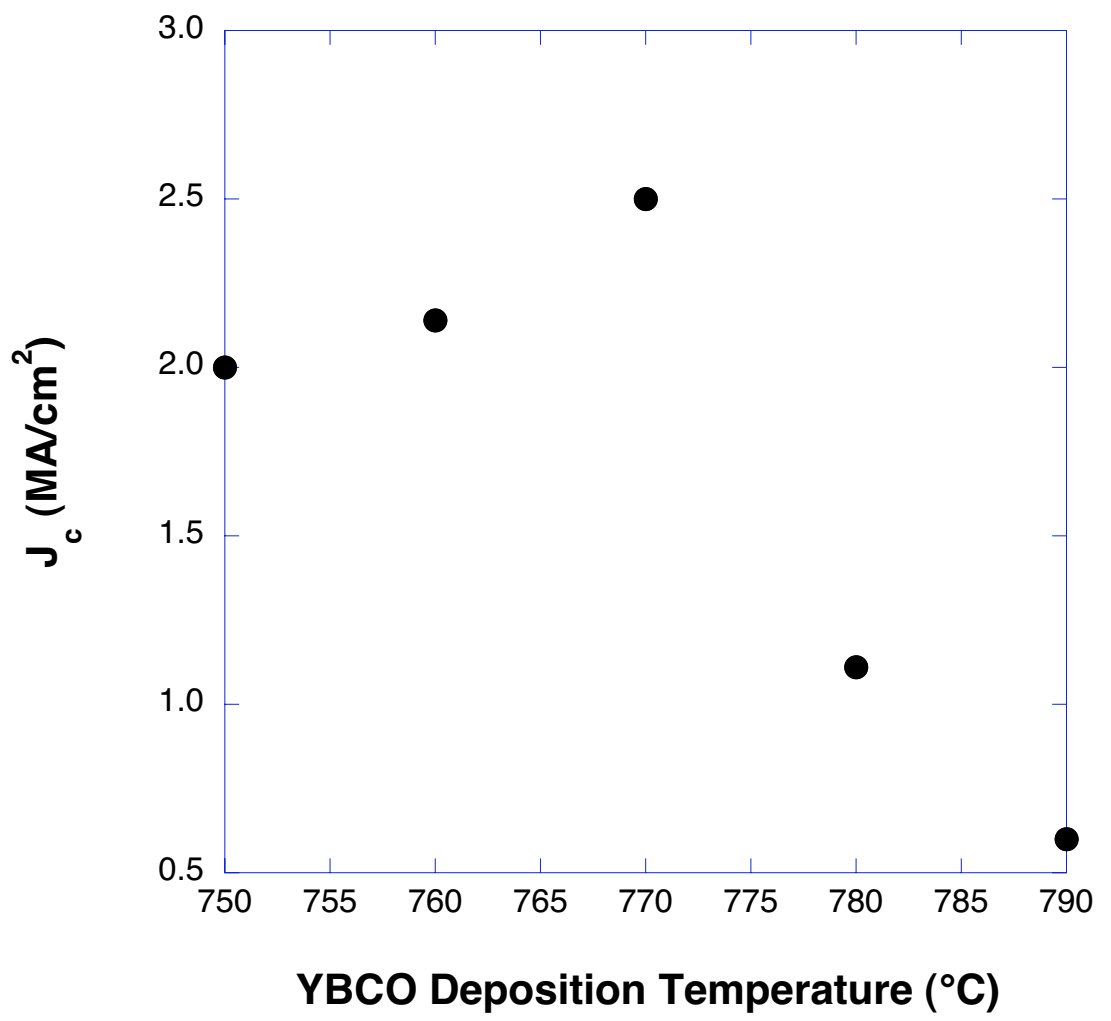

Fig. 20. Transport $J_{c}$ for YBCO films deposited by PLD on SRO-buffered single crystal $\mathrm{MgO}$ substrates versus temperature of YBCO deposition. 
The surface morphologies of the $\mathrm{YBCO}$ and SRO films were examined using SEM. Figure 21 shows an SRO film deposited at $770^{\circ} \mathrm{C}$ on single-crystal $\mathrm{MgO}$. The film has a smooth surface with well-connected grains; no large droplets are evident. SRO films deposited at 750,760 , and $780^{\circ} \mathrm{C}$ exhibit similar surface morphologies. The SRO film deposited at $790^{\circ} \mathrm{C}$, however, had larger grains than did the SRO films produced at other temperatures, and it contained numerous voids, as seen in Fig. 21d. The YBCO film deposited at $770^{\circ} \mathrm{C}$ on SRObuffered $\mathrm{MgO}$, shown in Fig. 22, contained uniform, apparently well connected YBCO grains along with a sprinkling of droplets $(\approx 1 \mu \mathrm{m}$ in diameter) that were probably ejected from the target during laser ablation. The film also contained numerous small grains that appear like a-axis oriented grains, an observation that is consistent with the Raman results presented later.
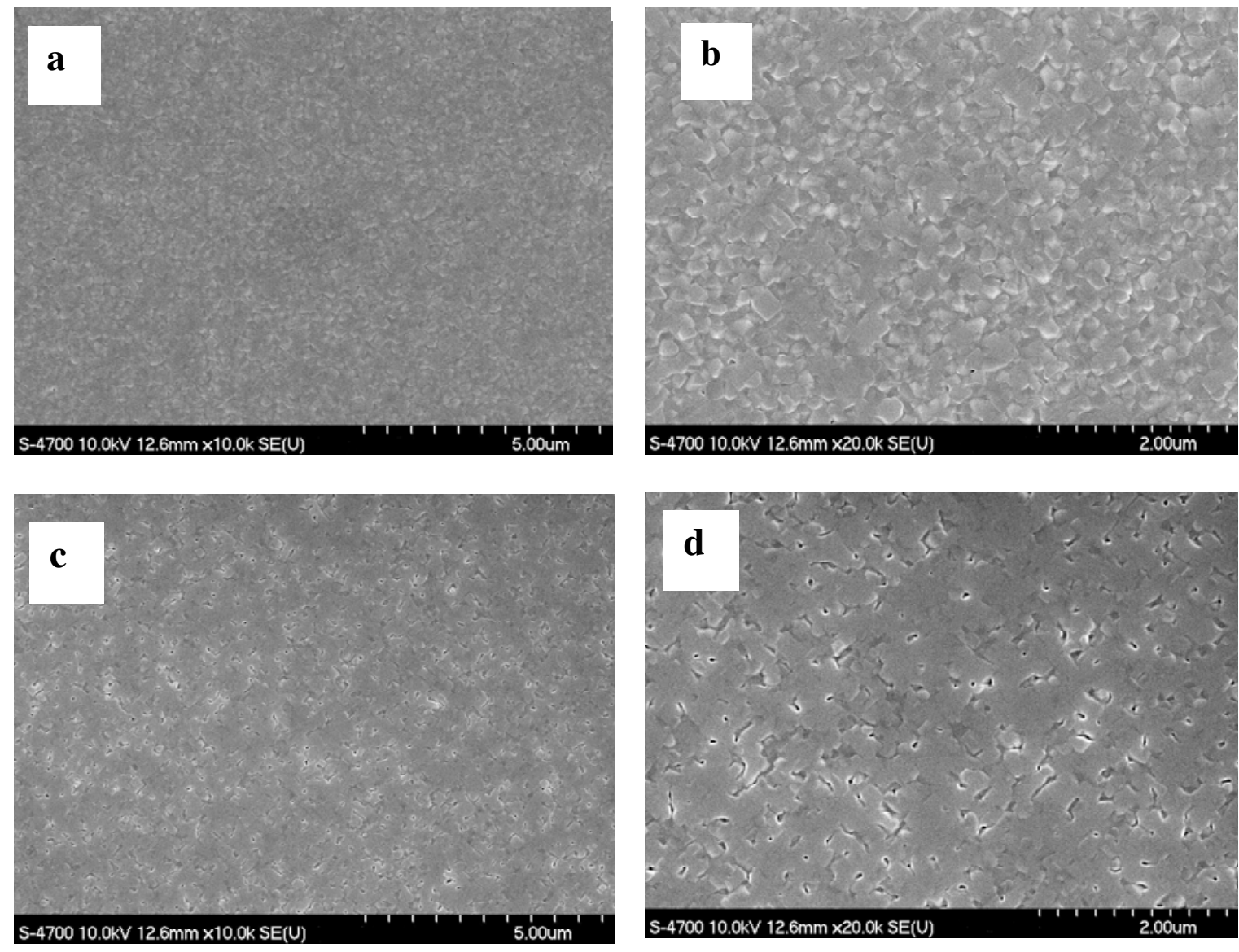

Fig. 21. Secondary electron images of SRO deposited by PLD on $\mathrm{MgO}$ single crystal substrate at: (a) $770^{\circ} \mathrm{C}$ at "low" magnification, (b) $770^{\circ} \mathrm{C}$ at "high" magnification, (c) $790^{\circ} \mathrm{C}$ at "low" magnification, and (d) $790^{\circ} \mathrm{C}$ at "high" magnification. 

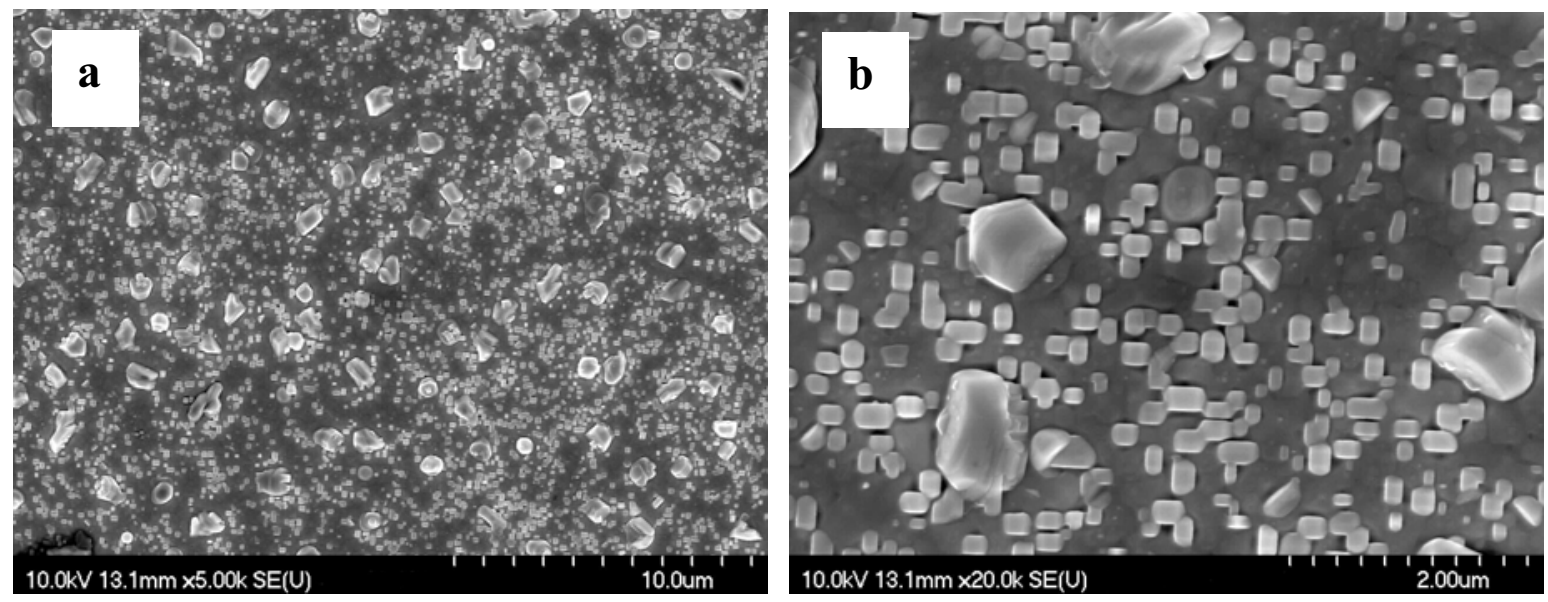

Fig. 22. Secondary electron images of $Y B C O$ deposited by $P L D$ at $770^{\circ} \mathrm{C}$ on SRO-buffered $\mathrm{MgO}$ single crystal substrate.

The YBCO films fabricated at 770 and $790^{\circ} \mathrm{C}$, respectively, were analyzed by Raman microspectroscopy using $632.82 \mathrm{~nm}$ red light. The main features in their spectra (Fig. 23) were: (i) a peak at $150 \mathrm{~cm}^{-1}$ caused by the motion of $\mathrm{Cu}(2)$ along the c-axis, (ii) a peak at $338 \mathrm{~cm}^{-1}$ associated with out-of-phase bond-bending of $\mathrm{O}(2)$ and $\mathrm{O}(3)$, and (iii) a peak at $500 \mathrm{~cm}^{-1}$ generated by vibration of $\mathrm{O}(4)$ atoms toward $\mathrm{Cu}(1)$. The peak at $500 \mathrm{~cm}^{-1}$ indicates that some of the YBCO grains are aaxis oriented or tilted from the vertical to the substrate.

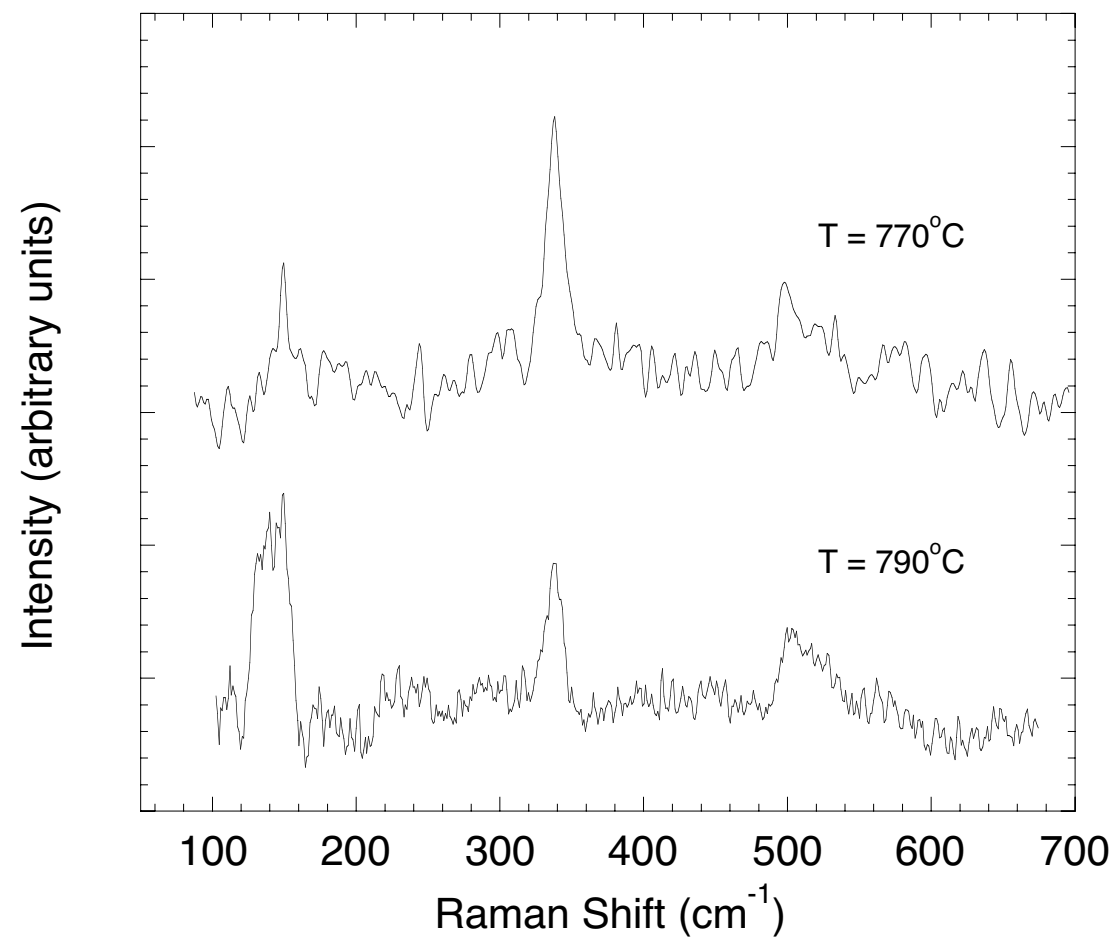

Fig. 23. Raman spectra of $Y B C O$ deposited on SRO-buffered $\mathrm{MgO}$ single crystal substrate at a) $770^{\circ} \mathrm{C}$, and b) $790^{\circ} \mathrm{C}$. 
The Raman spectra in Fig. 23 also showed a weak peak at $\approx 225 \mathrm{~cm}^{-1}$ (more intense in the case of the film deposited at $790^{\circ} \mathrm{C}$ ), indicating broken $\mathrm{M}-\mathrm{O}$ chain structures that can exist on a nanoscopic, microscopic, or macroscopic scale. The YBCO films consisted mainly of orthogonal (I) domains, as indicated by the positioning of the $\mathrm{Cu}(2)$ band at $150 \mathrm{~cm}^{-1}$. Both YBCO films appeared to be well oxygenated as indicated by the occurrence of the $\mathrm{Cu}(2)$ peak at $150 \mathrm{~cm}^{-1}$. According to these Raman results, the major difference between the films was in their degree of c-axis misalignment, as quantified by the average intensity ratio of the $\mathrm{I}_{\mathrm{O}(4)} / \mathrm{I}_{\mathrm{O}(2,3)}$ modes, which should be zero for a film that has perfect out-ofplane texture. Analysis showed that the $\mathrm{YBCO}$ film fabricated at $770^{\circ} \mathrm{C}$ had better out-of-plane texture $\left(\mathrm{I}_{\mathrm{O}(4)} / \mathrm{I}_{\mathrm{O}(2,3)}=1.5\right)$ than the film deposited at $790^{\circ} \mathrm{C}$, $\left(\mathrm{I}_{\mathrm{O}(4)} / \mathrm{I}_{\mathrm{O}(2,3)}=2.3\right)$, which might partially explain the higher transport $\mathrm{J}_{\mathrm{c}}$ for $\mathrm{YBCO}$ deposited at $770^{\circ} \mathrm{C}$.

\subsection{2.c SRO-Buffered ISD MgO Substrates}

After refining the conditions for the PLD of YBCO and SRO, we focused on the fabrication of YBCO films on SRO-buffered ISD substrates. HC substrates were mechanically polished to a mirror finish by using $0.25-\mu \mathrm{m}$ diamond paste. On the polished $\mathrm{HC}$ substrate, an ISD MgO film was deposited at an angle of $55^{\circ}$ from the substrate normal by electron beam evaporation. On top of the ISD film, a $\mathrm{HE} \mathrm{MgO}$ layer was deposited normal to the substrate. Hereafter, ISD MgO refers to the ISD and HE MgO layers together. An SRO film (thickness $\approx 100 \mathrm{~nm}$ ) was deposited on the ISD-MgO substrate by PLD at $770^{\circ} \mathrm{C}$ with an oxygen pressure of 50 mtorr. YBCO was then deposited on the SRO-buffered ISD MgO substrate at $760^{\circ} \mathrm{C}$ and 180 mtorr oxygen pressure. YBCO films were made with a thickness of $300 \mathrm{~nm}$ or $450 \mathrm{~nm}$. After the YBCO layer was deposited, it was annealed at $450^{\circ} \mathrm{C}$ for $90 \mathrm{~min}$ at an oxygen pressure of 750 torr.

Figure 24 shows an X-ray diffraction (XRD) pattern of YBCO on an SRObuffered ISD MgO substrate. The peaks that correspond to (00l) Miller indices ( $l$ $=1,2,3,4,5,6)$ are very strong, showing that the film was highly textured, with a strong $c$-axis orientation. An $\omega$-scan of the $\mathrm{YBCO}(005)$ peak gave a full width at half-maximum (FWHM) of $3.76^{\circ}$, showing good out-of-plane texture. $\phi$-scans of the $\mathrm{SRO}(020)$ and $\mathrm{MgO}(200)$ reflections (Fig. 25) gave FWHM's of $7.38^{\circ}$ for $\mathrm{SRO}(020)$, and $\approx 10^{\circ}$ for $\mathrm{MgO}(200)$; a $\phi$-scan of the $\mathrm{YBCO}(103)$ peak (Fig. 26) had a FWHM of $8.69^{\circ}$, indicating good in-plane texture. The YBCO(103) FWHM was found as low as $6.4^{\circ}$ for other films. 


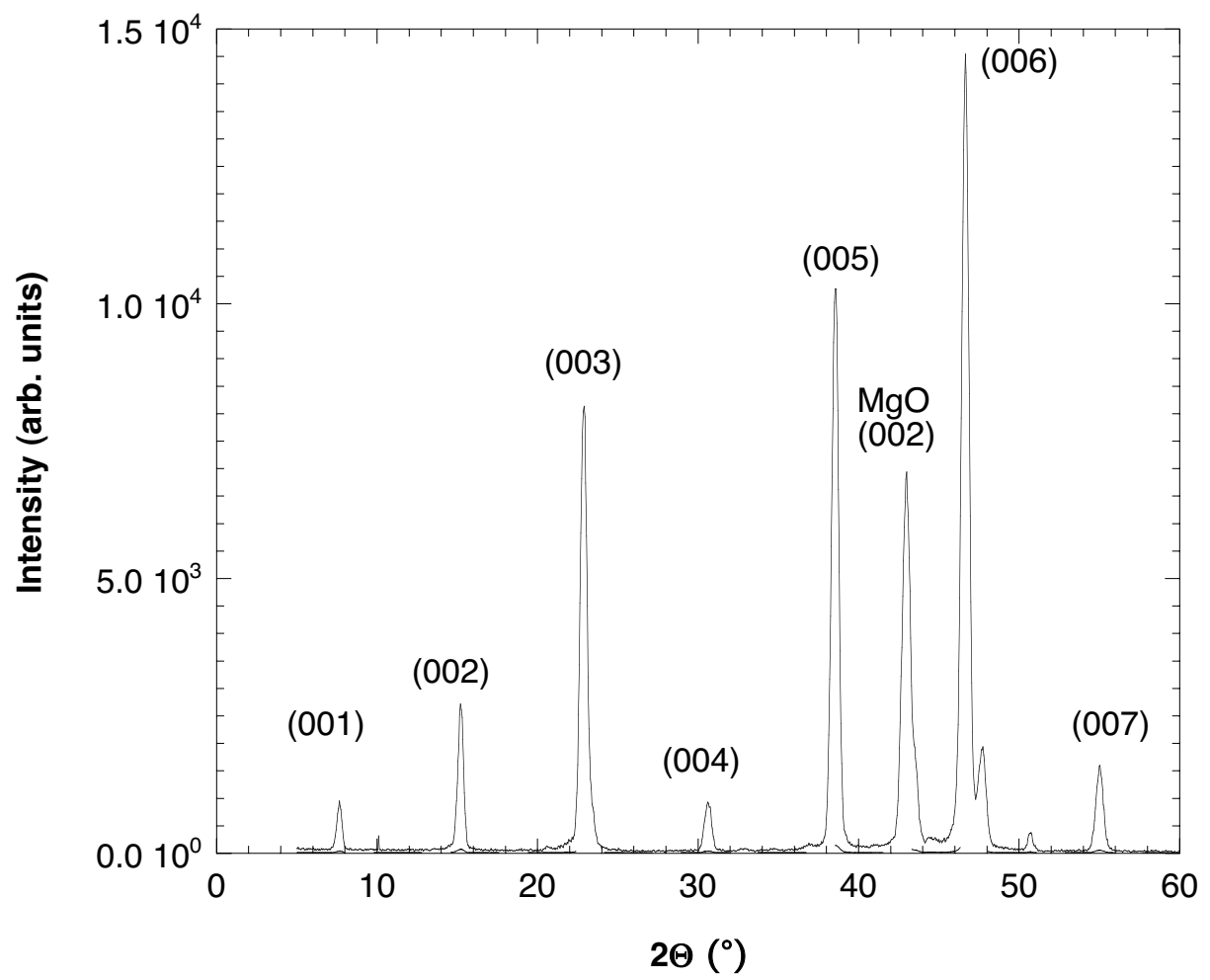

Fig. 24. X-ray diffraction pattern of YBCO/SRO/ISD MgO/HC structure.

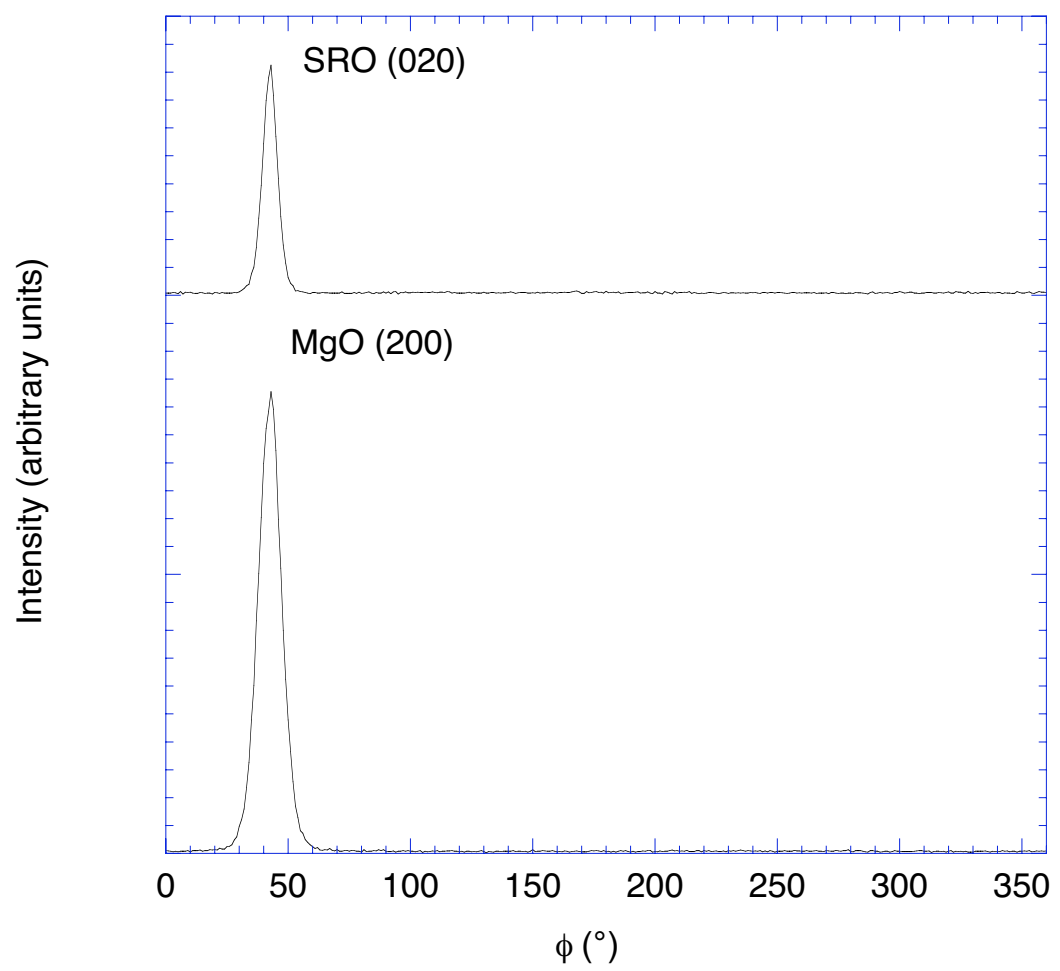

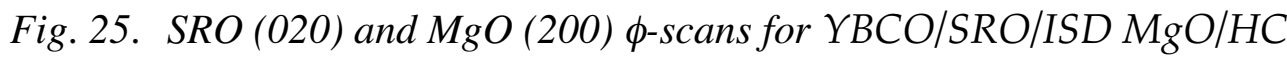
structure. 


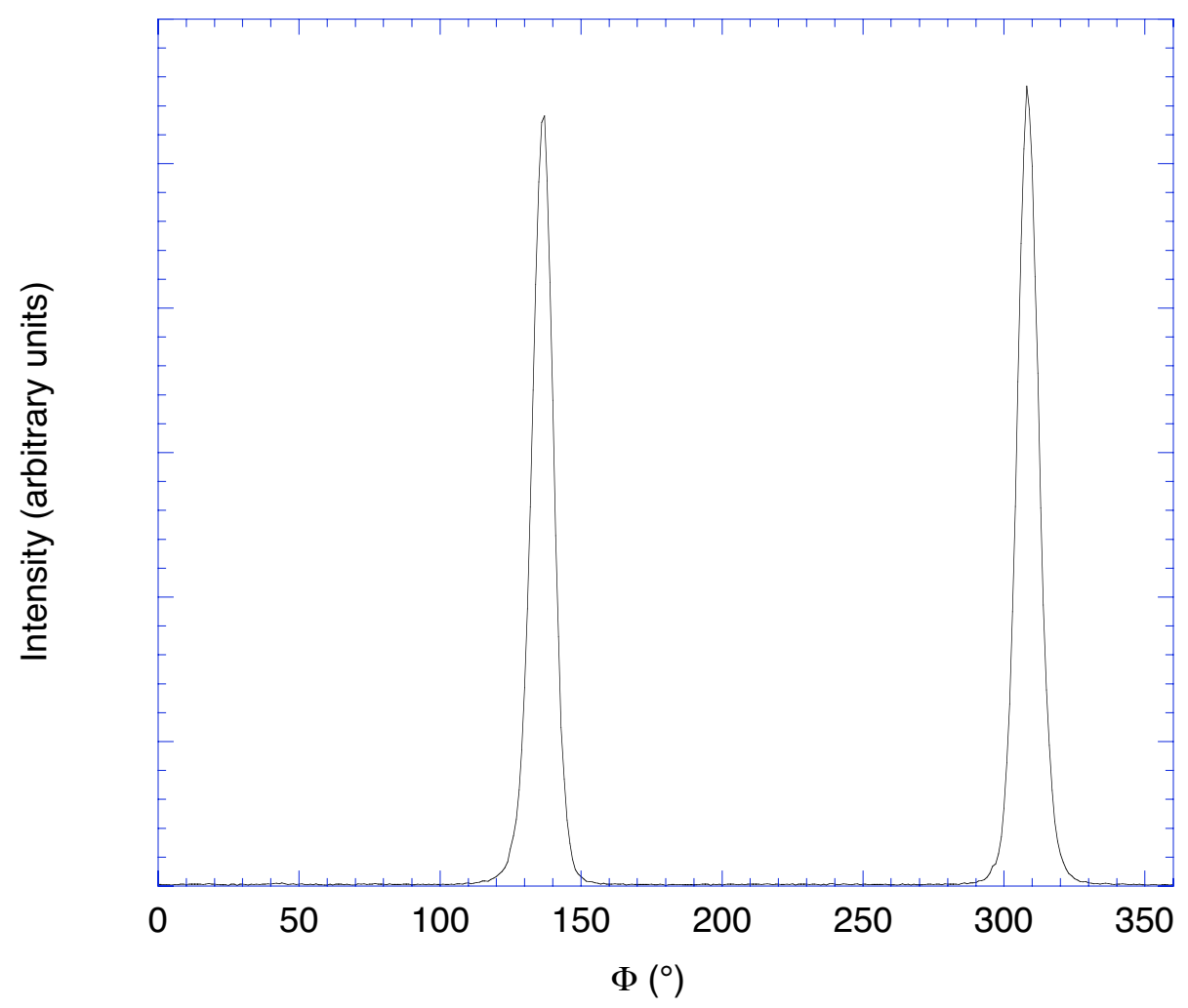

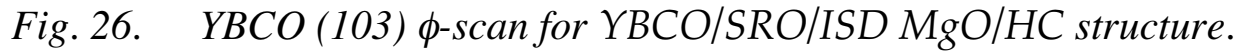

The $\mathrm{T}_{\mathrm{c}}$ of $0.3-\mu \mathrm{m}$-thick YBCO on SRO-buffered ISD MgO templates was measured by the inductive method described above; the $\mathrm{J}_{\mathrm{C}}$ was measured by the four-probe method against a $1-\mu \mathrm{V} / \mathrm{cm}$ criterion. Figure 27 shows that the $\mathrm{J}_{\mathrm{C}}$ of YBCO was $280 \mathrm{kA} / \mathrm{cm}^{2}$ at $77 \mathrm{~K}$ and zero applied magnetic field. Although this result indicated some improvement in transport properties, relative to earlier results on SRO-buffered ISD templates, Fig. 28 shows that the film exhibited a relatively low $\mathrm{T}_{\mathrm{C}}$ (onset) of $\approx 87 \mathrm{~K}$. This indicates that the $\mathrm{YBCO}$ deposition conditions need further refinement. This conclusion is consistent with scanning electron microscopy/energy dispersive spectroscopy (SEM/EDS) results that show the composition of the YBCO film is not completely homogeneous. 


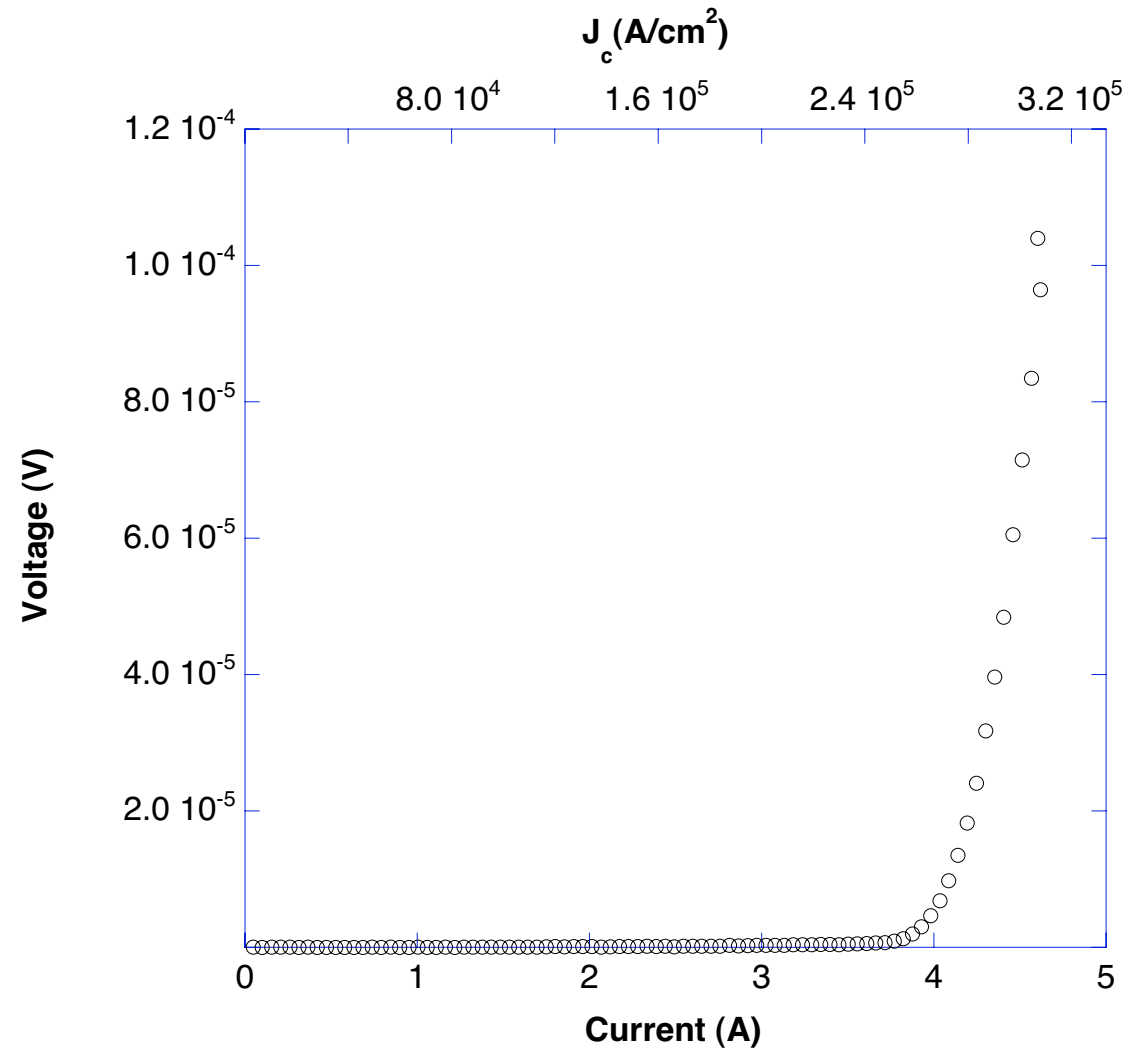

Fig. 27. Transport critical current density $\left(J_{c}\right)$ of $Y B C O / S R O / I S D$ $\mathrm{MgO} / \mathrm{HC}$ structure.

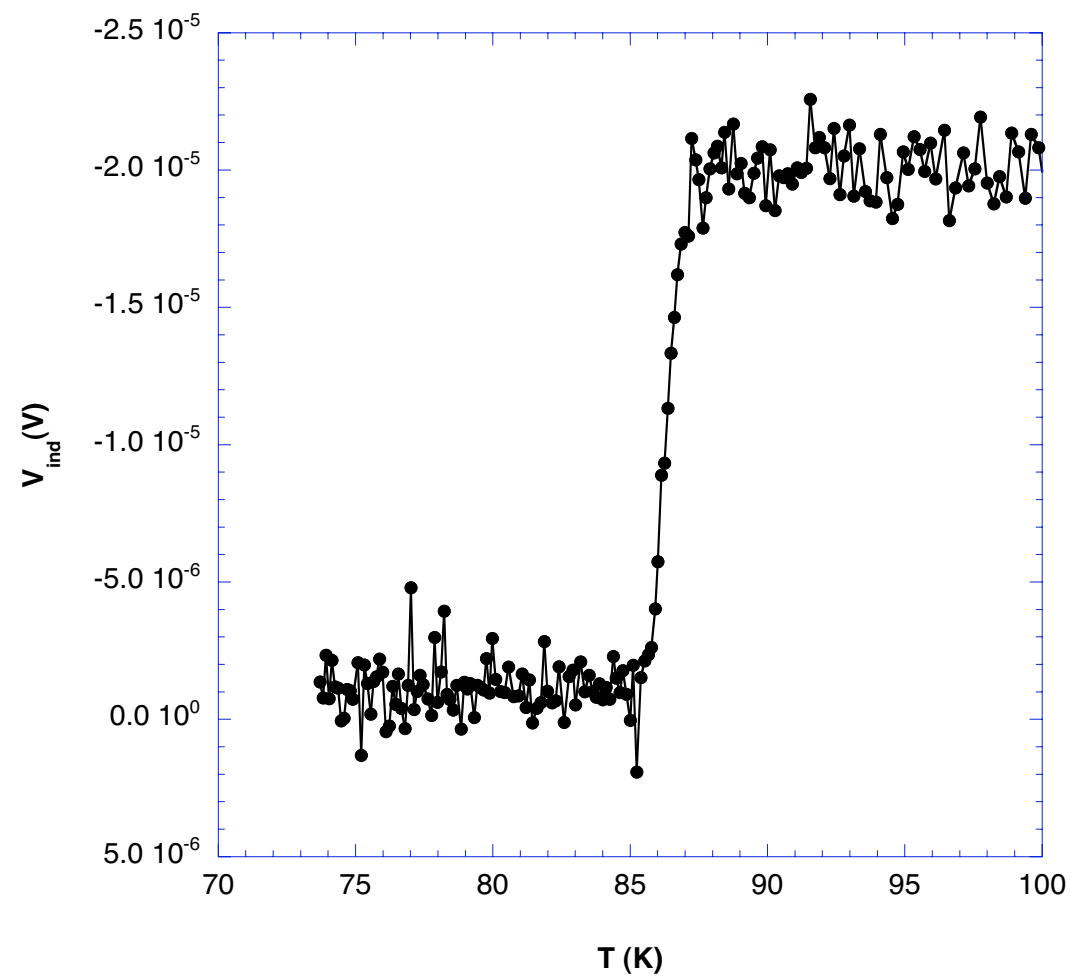

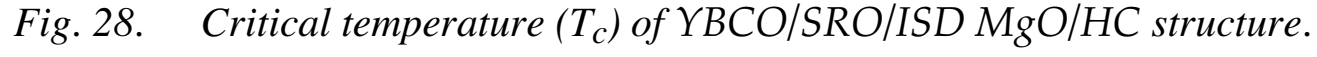




\subsubsection{Direct Deposition of YBCO on Ag Alloy Substrates}

During FY 2003, we continued investigating the PLD of YBCO on Ag-Cu alloy substrates without any intermediate buffer layers. We reported earlier [3] that the performance of $\mathrm{YBCO}$ was greatly enhanced in conductors made with Ag substrates that contained $\mathrm{Cu}$, presumably because the $\mathrm{Cu}$ additions reduced the driving force for $\mathrm{Cu}$ diffusion from $\mathrm{YBCO}$ into the substrate and helped to preserve the correct $\mathrm{YBCO}$ stoichiometry at the $\mathrm{YBCO} /$ substrate interface. Furthermore, our earlier study showed that $\mathrm{Ag}$ substrates with the optimum $\mathrm{Cu}$ content $(\approx 0.2$ at. $\% \mathrm{Cu})$ gave the best superconducting properties for the deposited YBCO films. These experiments were all done with as-rolled, polycrystalline Ag or Ag-Cu substrates with a $\{110\}<112>$ deformation texture. $X$-ray pole figure analysis on substrates quenched after various times at typical YBCO deposition conditions showed that the deformation texture quickly transformed into the $\{236\}<385>$ texture at the deposition temperature. Substrates with this texture, however, did not yield $\mathrm{YBCO}$ with a single in-plane orientation. Because it is reported $[4,5]$ that the $\{110\}<110>$ and $\{110\}<112>$ textures are the most likely to produce $\mathrm{YBCO}$ with a single in-plane orientation, we studied the heat-treatment of Ag-Cu substrates in FY 2003 to identify the conditions under which these textures are stable.

The desired recrystallization textures were obtained by identifying the proper annealing conditions (temperature, time and atmosphere). $\{110\}<110>$ recrystallization texture with a weak secondary texture was obtained by coldrolling in air Ag- 0.2 at.\% $\mathrm{Cu}$ (containing dissolved oxygen) to a total thickness reduction of $90 \%$ (5\% reduction per pass) and then annealing it at $700^{\circ} \mathrm{C}$ for $4 \mathrm{~h}$ in $1 \mathrm{~atm}$ Ar. Figures 29a and 29b show the (111)- and (220)-pole figures, respectively, for this texture. $\{110\}<112>$ texture was obtained by a two-step annealing procedure; the (111)- and (220)-pole figures for this texture are shown in Figs. 29c and 29d, respectively. First, oxygen-free material was cold-rolled in air at room temperature to $97 \%$ total thickness reduction (5\% reduction per pass, annealed for $30 \mathrm{~min}$ in air at $500^{\circ} \mathrm{C}$ and then quenched in water. Second, the substrate was annealed again for $1 \mathrm{~h}$ in air at $800^{\circ} \mathrm{C}$ and quenched in water.

$X R D$ studies revealed that both textures were stable under the conditions used for YBCO deposition. When we deposited YBCO on these textured substrates, however, the YBCO showed no evidence of in-plane alignment, and the film's superconducting properties were poorer than those obtained for YBCO fabricated on as-rolled Ag-Cu substrates. The lack of in-plane alignment and poor properties are believed to result from grain boundary grooving and grain faceting in the $\mathrm{Ag}-\mathrm{Cu}$ substrates at temperatures above $200^{\circ} \mathrm{C}$. Figure 30 shows the grain boundary grooving and grain faceting that were present in a silver 
substrate after it was annealed $25 \mathrm{~min}$ at $780^{\circ} \mathrm{C}$ in $290 \mathrm{mtorr} \mathrm{O}_{2}$. Grain boundary grooving and grain faceting produce a distribution of $\mathrm{YBCO}$ orientations, because $\mathrm{YBCO}$ grains tend to grow with their c-axes normal to the $\mathrm{Ag}-\mathrm{Cu}$ surface.

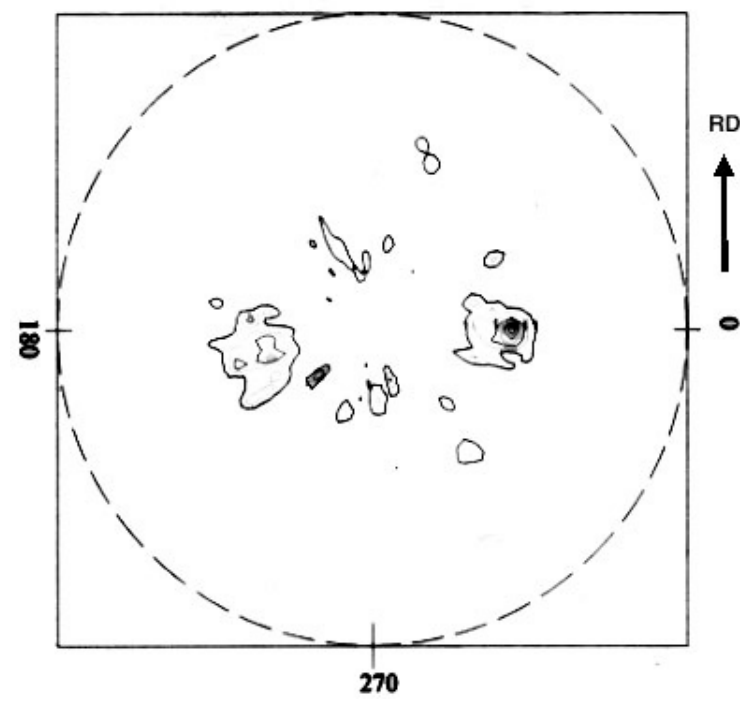

(a)

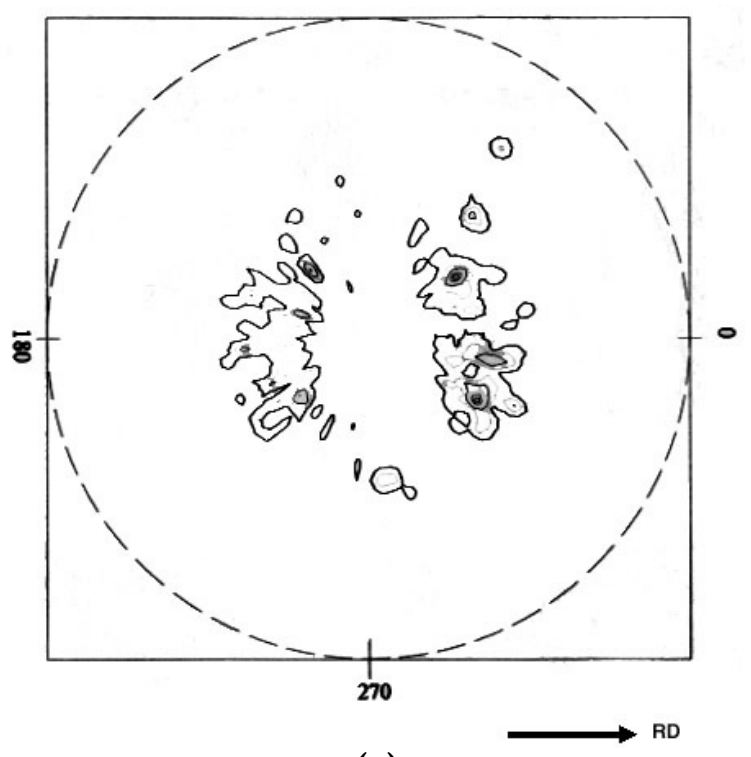

(c)

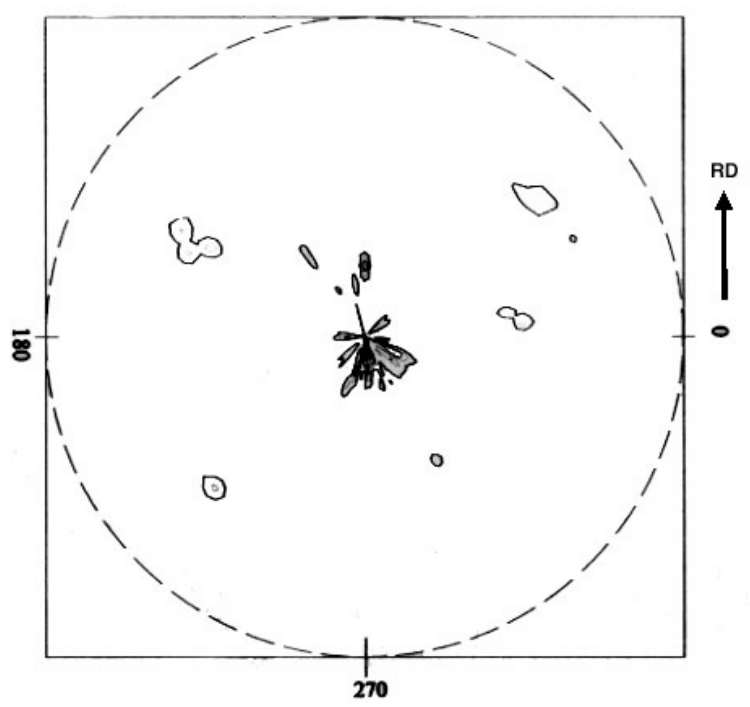

(b)

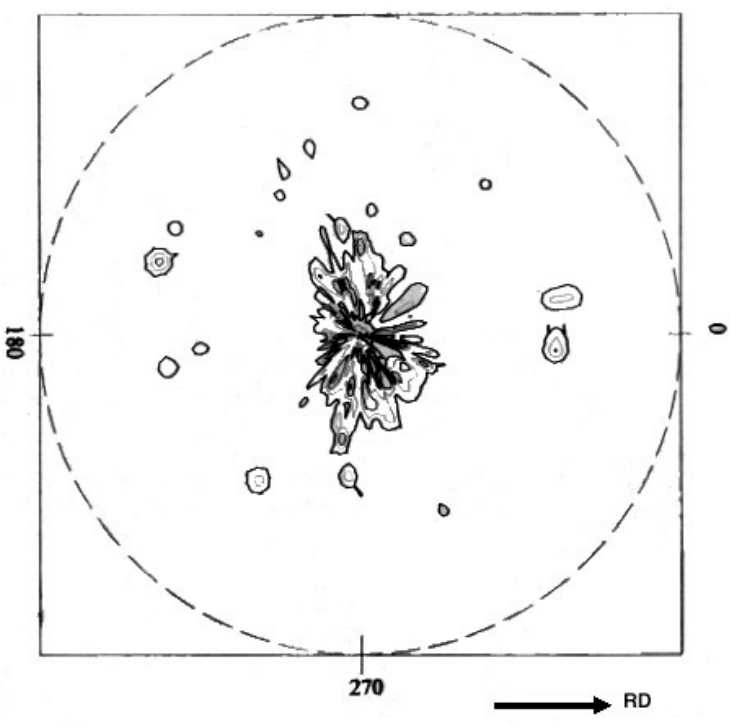

(d)

Fig. 29. Pole figures of Ag-0.2 at.\% Cu substrates with $\{110\}<110>$ and $\{110\}<112>$ textures: (a) (111) pole figure for $\{110\}<110>$ texture, (b) (220) pole figure for $\{110\}<110>$ texture, (c) (111) pole figure for $\{110\}<112>$ texture, $(d),(220)$ pole figure for $\{110\}<112>$ texture. 


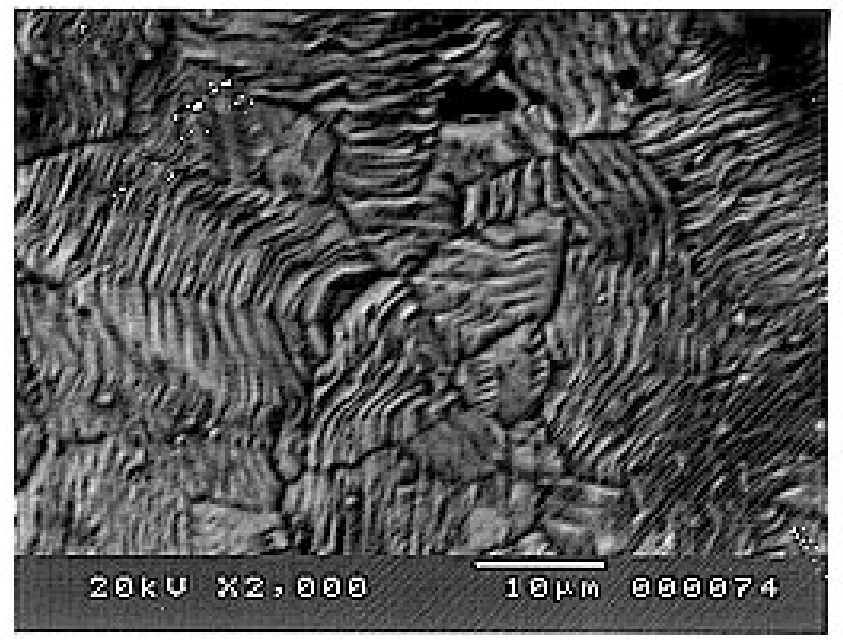

Fig. 30. Thermal faceting and grain boundary grooving in a $\mathrm{Ag}-\mathrm{Cu}$ alloy substrate that was exposed to PLD characteristic temperatures.

Grain boundary grooving in the Ag-Cu substrate may also be connected to formation of waviness in the YBCO grain boundary, as well as accumulation of voids and particles (e.g., $\mathrm{CuO}$ ) at the $\mathrm{YBCO}$ grain boundaries. Figure 31 shows examples of the particles (Fig. 31a) and voids (Fig. 31b) that have been seen in YBCO films that were deposited on recrystallized $\mathrm{Ag} / \mathrm{Cu}$ substrates. All these factors probably account for the poor performance exhibited by YBCO films that were fabricated on textured Ag-Cu substrates. A Ag-Cu substrate that was annealed in Ar, however, showed (Fig. 32) very few of the features that were evident in substrates annealed in air (Fig. 30), suggesting that using an Ar atmosphere during recrystallization and while heating to the YBCO deposition temperature may inhibit grain boundary grooving and grain faceting in $\mathrm{Ag}-\mathrm{Cu}$ substrates.

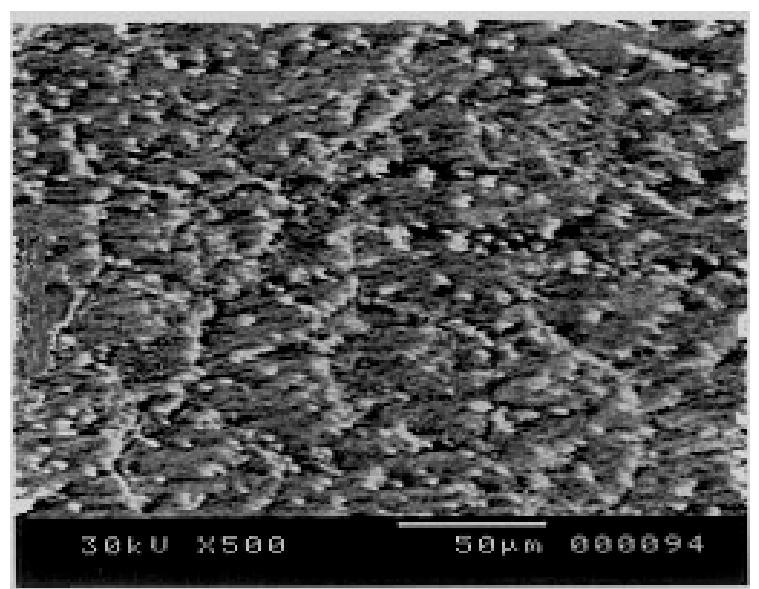

(a)

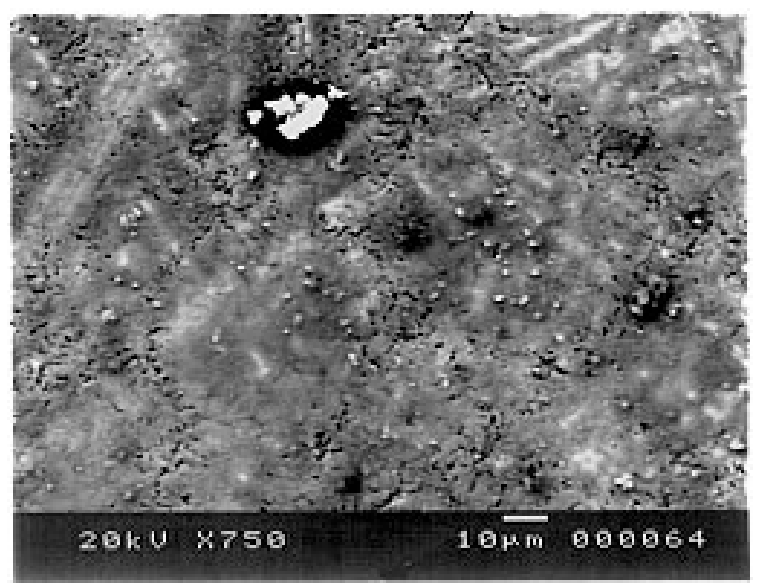

(b)

Fig. 31. Deleterious features in YBCO films on Ag-Cu substrates recrystallized in oxygen-containing atmosphere: (a) particles, and (b) voids. 


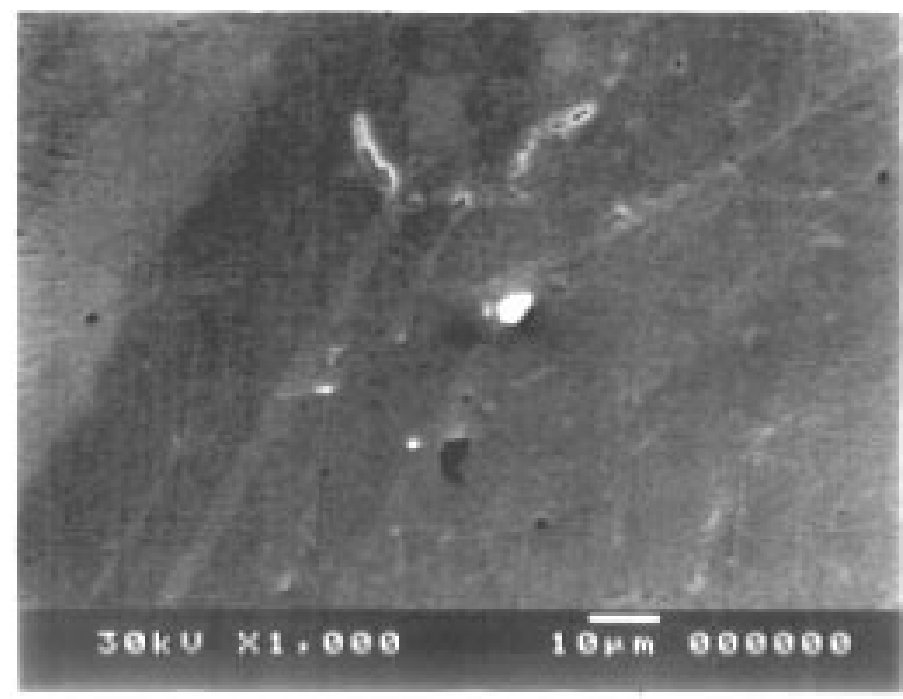

Fig. 32. SEM image of Ag-Cu substrate after it was annealed at $780^{\circ} \mathrm{C}$ in $1 \mathrm{~atm} \mathrm{Ar}$.

\subsubsection{Raman Microscopy of Coated Conductors}

\subsection{4.a Studies Probing Applicability of Raman Microscopy}

Beginning in FY 2002 and throughout FY 2003, we studied the usefulness of Raman microscopy methods in investigating phase composition, cation disorder, and texture quality of coated-conductors. This study had three objectives in mind: (1) developing an improved understanding of the methodology itself (e.g., in terms of how Raman excitation/configuration parameters influence the observations), (2) investigating systematically prepared groups of samples to provide meaningful correlations with process parameters and with superconductor performance levels (including substantial collaboration with other national laboratories and industrial partners), and (3) examining the applicability of Raman microscopy as an on-line process-monitoring tool during fabrication of long-length coated conductors. We summarize here key results from this initial effort to use Raman microscopy to characterize $\mathrm{MBa}_{2} \mathrm{Cu}_{3} \mathrm{O}_{\mathrm{x}}$ ( $\mathrm{MBCO}$, where $\mathrm{M}=\mathrm{Y}$ or a rare earth element) thin films on textured substrates.

One of the issues involved in interpreting the Raman microscopy results has been the matter of laser penetration depth, i.e., what portion of the sample is being interrogated. Figure 33 shows the results of Raman microprobe measurements on three EuBCO films of varying thickness deposited by pulsed laser deposition (PLD) on La-Sr-Al-Ta-O (LSAT) substrates. This series of samples, prepared by Q. Jia at Los Alamos National Laboratory (LANL), consisted of uniformly deposited EuBCO films, free of cracks, voids, and other imperfections that might allow the laser to strike bare substrate. Employing a 633 -nm laser excitation wavelength at a surface power density of $\approx 0.2 \mathrm{~mW}$ per 
$\mu \mathrm{m}^{2}$, and using the LSAT phonon at $\approx 470 \mathrm{~cm}^{-1}$ as a guide, we found (see Fig. 33) that the LSAT was readily detectable at an EuBCO thickness of $0.19 \mu \mathrm{m}$, barely detectable at a thickness of $0.3 \mu \mathrm{m}$, and undetectable at a thickness of $0.5 \mu \mathrm{m}$. We have concluded from this study that for the excitation conditions we typically use to investigate $\mathrm{MBCO}$ films, the depth below the $\mathrm{MBCO}$ surface from which detectable Raman scattering emanates is approximately $0.3 \mu \mathrm{m}$.

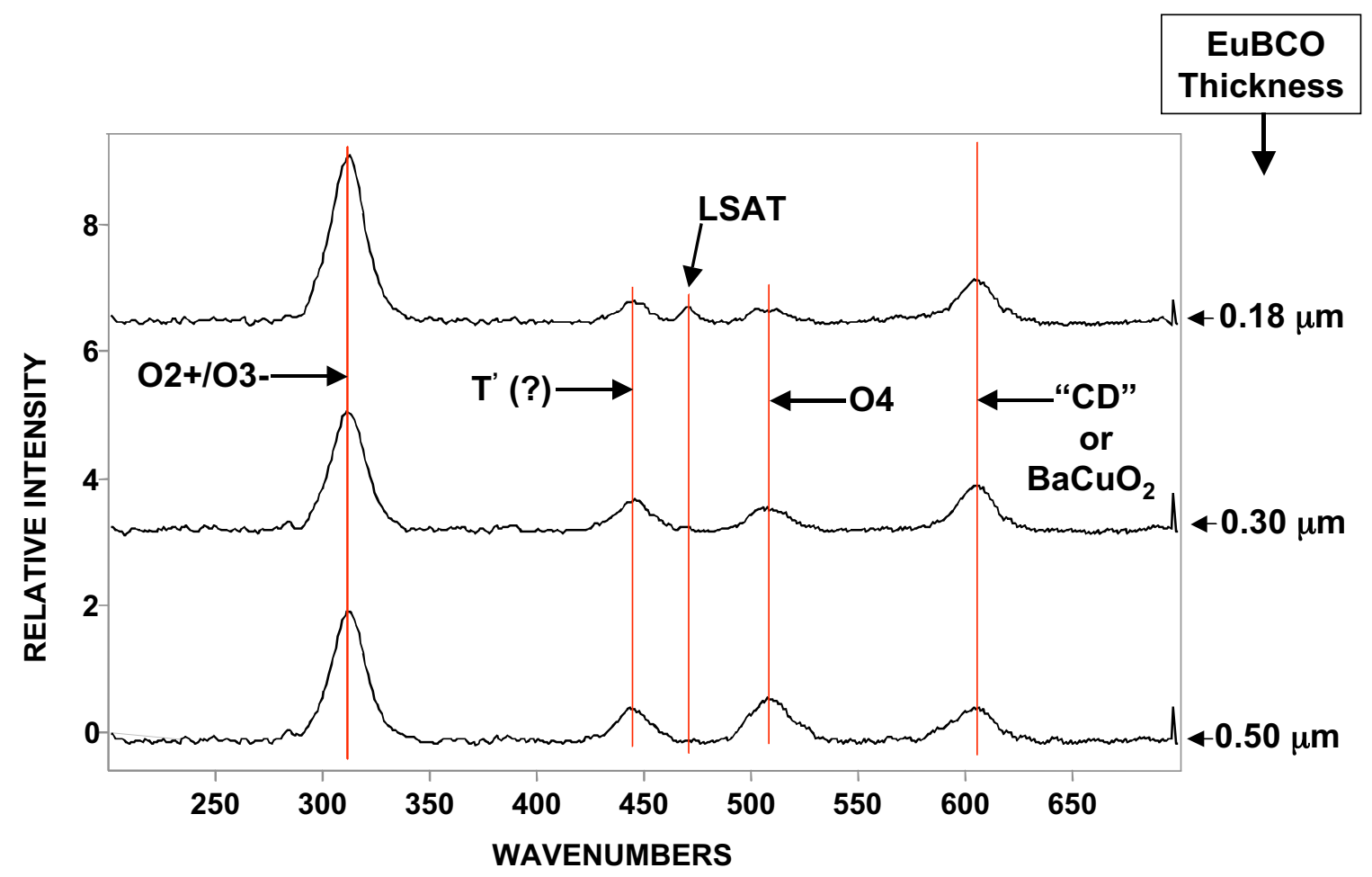

Fig. 33. Raman microprobe spectra for three thicknesses of PLD-type EuBCO on single-crystal La-Sr-Al-Ta-O (LSAT) substrates. "CD" represents cation disorder.

Figure 33 illustrates most of the informative features that appear in typical Raman spectra of the MBCO films we have studied thus far. These features include (1) the $\mathrm{O} 2+/ \mathrm{O} 3-$ and $\mathrm{O} 4$ phonons of orthorhombic $\mathrm{MBCO}$, the relative intensities of which provide information about the c-axis tilt associated with the presence of "a-axis" grains or tilted MBCO grains (as discussed in previous quarterly reports), (2) modes that are associated with cation disorder and/or the presence of barium cuprate second phases, and (3) the mode at $\approx 450 \mathrm{~cm}^{-1}$, which indicates the presence of residual tetragonal MBCO domains.

Figure 34 shows the results of a study conducted to investigate the influence of laser excitation wavelength on the observed Raman spectra of YBCO films of varying thickness. These samples, provided by A. Goyal of Oak Ridge National Laboratory (ORNL), consist of PLD YBCO on single-crystal $\mathrm{SrTiO}_{3}$ 
(STO). The spectrum of the $0.19-\mu \mathrm{m}$-thick film, taken with $476-\mathrm{nm}$ excitation, exhibits broad underlying background features centered near $330 \mathrm{~cm}^{-1}$ and 700 $\mathrm{cm}^{-1}$ that are due to resonance enhancement of the second-order Raman spectrum of STO. Note that this enhancement is not seen with 633-nm excitation. For the thicker YBCO specimen, the spectra obtained with 633- and 476-nm excitation are qualitatively similar in terms of the observed spectral features and the signalto-noise level. The reduced intensity of the $\mathrm{O} 2+/ \mathrm{O} 3-$ mode relative to the $\mathrm{O} 4$ mode with 476-nm excitation is attributed to less penetration of the YBCO when compared with the case for 633-nm excitation, and is most probably a consequence of the presence of more "a-axis" YBCO near the surface of the film than in the bulk. In essence, the 633-nm excitation penetrates deeper into the film (where there is less "a-axis" grain development) than does the 476-nm excitation. We find that, in general, 633-nm excitation is superior to 476-nm excitation for measurements on MBCO coated conductors, because it exhibits a lesser tendency to stimulate resonantly enhanced spectra and because it penetrates more deeply into the MBCO film. Similar comparative studies with 514-nm excitation revealed that laser wavelengths in the "green" part of the spectrum tend to preferentially excite the resonance enhanced Raman spectra of $\mathrm{BaCuO}_{2}$ without improving the penetrating ability of 633-nm radiation.

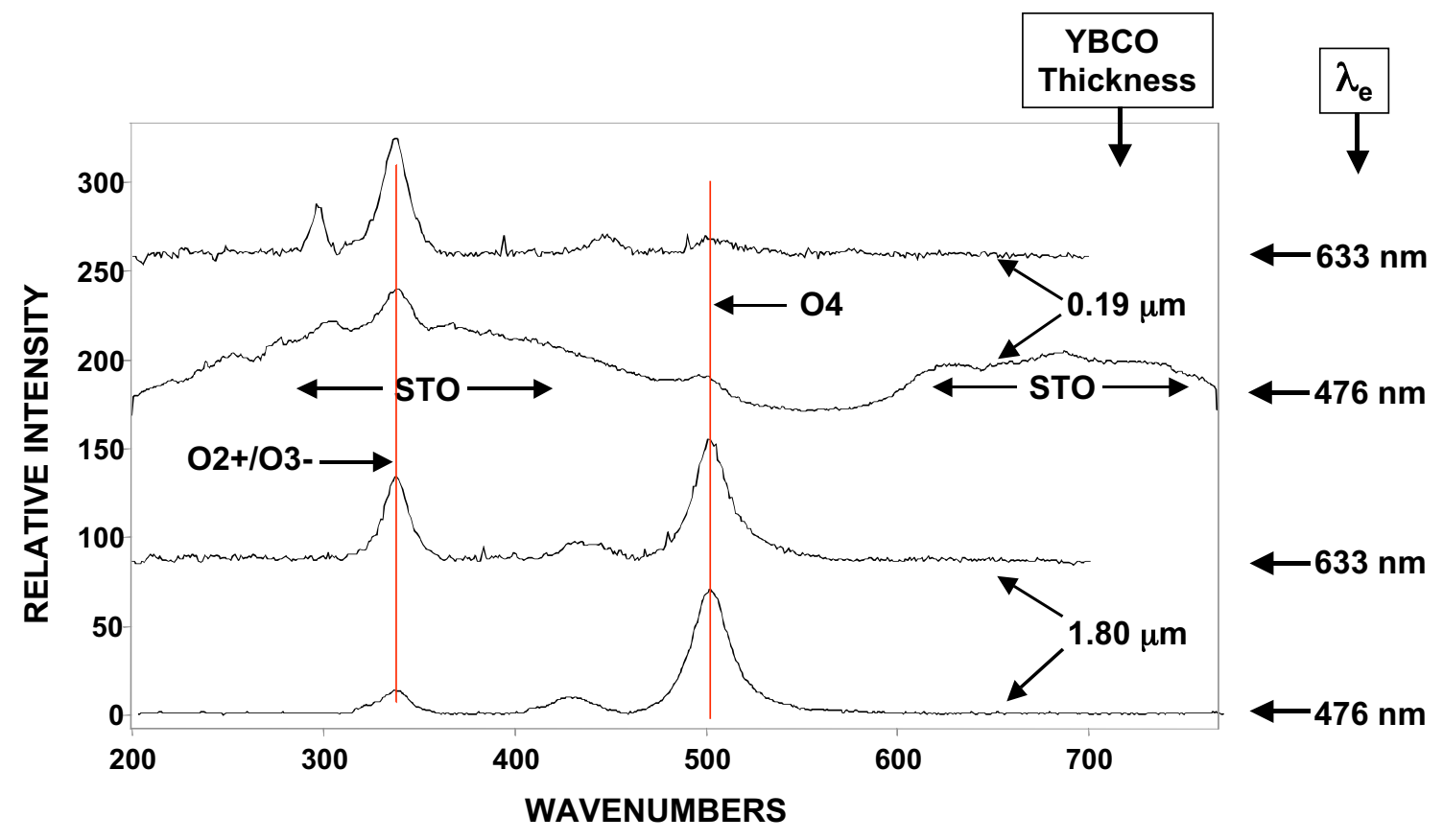

Fig. 34. Raman spectra of 0.19- and 1.80-um-thick PLD-type YBCO films on single-crystal $\mathrm{SrTiO}_{3}$ (STO) recorded with two laser excitation wavelengths $\left(\lambda_{e}=633\right.$ and $\left.476 \mathrm{~nm}\right)$. 
Observation over the past year of a band near $450 \mathrm{~cm}^{-1}$ in the Raman spectra of YBCO-coated conductor specimens from several collaborating institutions has prompted us to more thoroughly investigate its origin. To confirm our initial presumption that this mode is due to tetragonal YBCO domains within largely orthorhombic YBCO thin films (a circumstance commonly referred to as phase separation in connection with YBCO phase evolution), we examined several samples that contained this mode as a prominent spectral feature (samples provided by S. Foltyn at LANL ). In Fig. 35, we show spectra of two of these samples, both PLD YBCO on a buffered metal substrate (in one case IBAD YSZ/Hastelloy C, in the other, a Ni-RABiTS substrate provided by ORNL), together with the spectrum of a melt-processed YBCO sample known to be tetragonal YBCO (obtained from P. Diko at the Slovak Academy of Sciences). This observation calls attention to the possibility that oxygenation of YBCO films may, in some cases, be either incomplete or somehow inhibited during fabrication of coated conductor specimens. The extent to which this phase separation acts to degrade critical current density $\left(\mathrm{J}_{\mathrm{c}}\right)$ and/or contributes in a beneficial way to flux pinning remains to be determined.

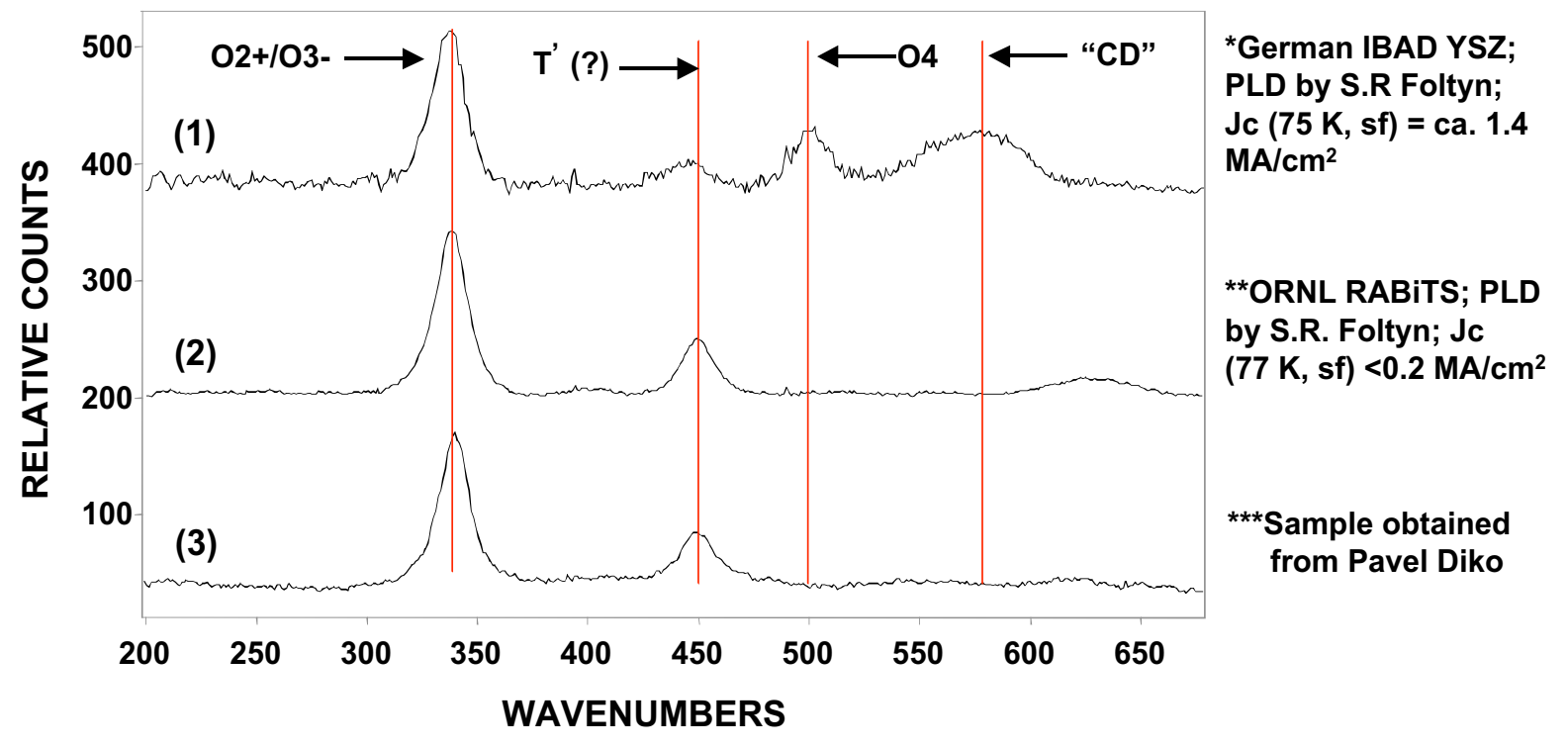

Fig. 35. Raman microprobe spectra of YBCO samples exhibiting the 470 $\mathrm{cm}^{-1}$ mode indicative of tetragonal form of $Y B C O$ : (1) $Y B C O$ (1.2 $\mu m$ by $P L D) / S m B C O(0.1 \mu m$ by PLD)/YBCO (1.2 $\mu m$ by PLD)/ $\mathrm{CeO}_{2} / Y S Z /$ Hastelloy $C^{*}{ }^{*}$ (2) YBCO (1.0 $\mu \mathrm{m}$ by $\mathrm{PLD}) / \mathrm{CeO}_{2} / \mathrm{YSZ} / \mathrm{CeO}_{2} / \mathrm{Ni}-\mathrm{RABiTS},{ }^{* *}$ (3) tetragonal YBCO bulk specimen (melt-processed/textured). ${ }^{* *}$ See notes in figure for additional details. 
In collaboration with $\mathrm{A}$. Goyal at ORNL, we performed Raman measurements on a series of PLD YBCO films of varying thickness deposited on STO to investigate changes in film composition and c-axis alignment as YBCO film thickness increased. The results of this study, shown in Fig. 36, indicate that the onset of tilted YBCO grain growth begins to occur at thickness values between 0.4 and $0.7 \mu \mathrm{m}$. (This is determined by the increase in intensity of the $\mathrm{O} 4$ mode of $\mathrm{YBCO}$ at $500 \mathrm{~cm}^{-1}$ relative the $\mathrm{O} 2+/ \mathrm{O} 3$ - mode at $340 \mathrm{~cm}^{-1}$.) In Fig. 36, it is noteworthy that the $\mathrm{J}_{\mathrm{c}}$ initially drops off with increasing film thickness but eventually reaches a relatively constant value at a YBCO thickness near $0.7 \mu \mathrm{m}$. In an independent investigation of these same samples using X-ray scattering methods, Kang et al. [6] also correlated this behavior with the progressive growth of "a-axis" grains within the c-axis films, as well as with a general broadening of the in-plane texture.

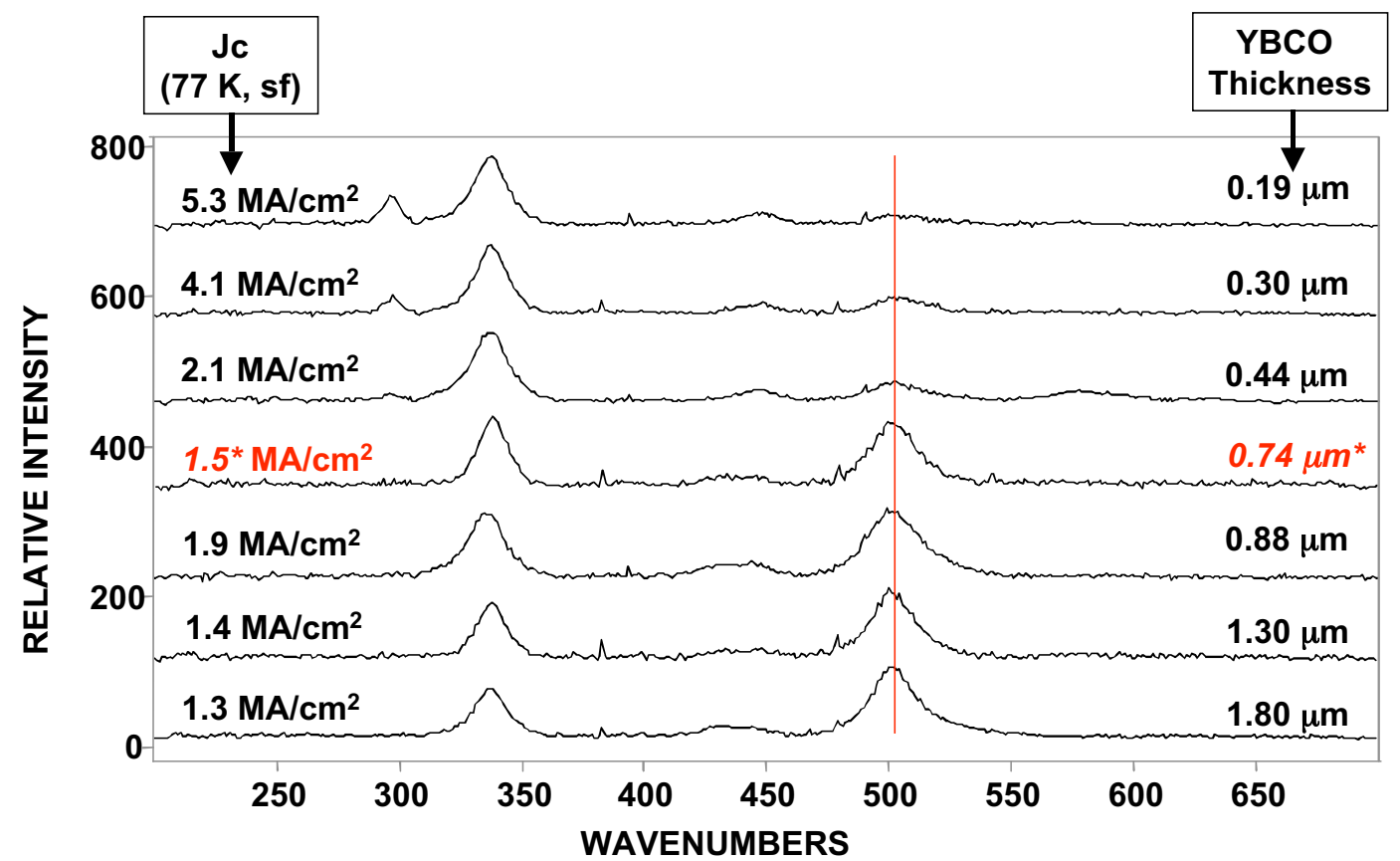

Fig. 36. Averaged Raman microprobe spectra of PLD-type YBCO films of varying thickness. Each plotted spectrum is the average of individual spectra taken at nine locations on specimen surface. Films with thickness $=0.19,0.30,0.44,0.88,1.30$, and $1.80 \mu \mathrm{m}$ are on singlecrystal STO substrates. Film with thickness $=0.74 \mu m^{*}$ is on singlecrystal $\mathrm{LaAlO}_{3}$.

The effect of "a-axis" grain growth on the texture quality of the YBCO films can be seen in Fig. 37, which shows the results of texture mapping performed with the Raman microprobe. In this mapping procedure, we collect Raman spectra at evenly spaced locations throughout the section of the film lying between the current leads used to measure electrical properties. From these 
spectra, we obtain values for the intensity (I) of the 500 and $340 \mathrm{~cm}^{-1}$ modes of YBCO, calculate the intensity ratio $\left(\mathrm{I}_{500} / \mathrm{I}_{340}\right)$, and use this ratio to create a contour map of the texture profile, as shown in Fig. 37. An increasing value of $I_{500} / I_{340}$ implies increasing "a-axis" grain presence. From the results in Fig. 37, it is clear that the through-thickness texture (assuming full penetration by the laser) of the thinner YBCO film $(0.19 \mu \mathrm{m})$ is much better than that of the outer $\approx 0.3 \mu \mathrm{m}$ layer of the thicker $(1.8 \mu \mathrm{m})$ film.

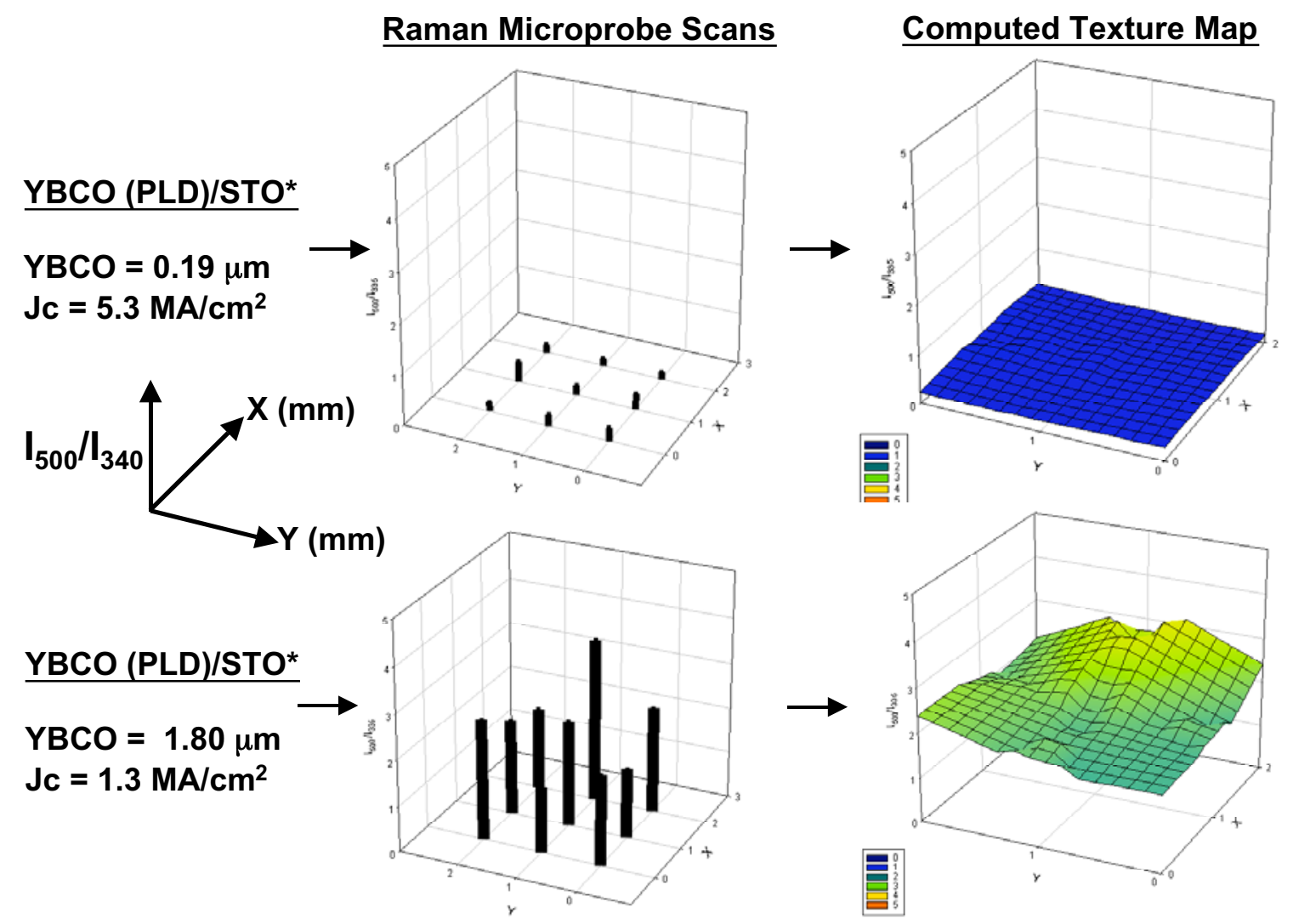

Fig. 37. Results of Raman microprobe texture mapping for 0.19- and 1.80um-thick PLD-type YBCO films on single crystal $\mathrm{SrTiO}_{3}$ (STO). Poles in scan plots indicate spatial locations and corresponding $I_{500} / I_{340}$ values. Texture maps show computer-generated textured maps based on $I_{500} / I_{340}$ values in scan plots.

In collaboration with R. Feenstra at ORNL, we began an investigation of the composition and texture quality of ex situ-type $\mathrm{YBCO}$ films produced by the $\mathrm{BaF}_{2}$ process. The $\mathrm{J}_{\mathrm{c}}$ values of ex situ YBCO films on buffered metal substrates are comparable to those of PLD films on the same substrate systems. Raman microprobe spectra for ex situ YBCO films of varying thickness deposited on LANL-produced $\mathrm{CeO}_{2} / \mathrm{IBAD}$ YSZ/Ni-alloy and ORNL-produced $\mathrm{CeO}_{2} / \mathrm{YSZ} / \mathrm{Y}_{2} \mathrm{O}_{3} / \mathrm{Ni}$-RABiTS substrates are shown in Fig. 38, together with the 
YBCO thickness and $J_{c}$ value for each specimen. From these studies, we found that the ex situ films contain a relatively small but measurable fraction of tilted YBCO grains due either to general topographic roughness or "a-axis" grain clusters. This can be seen in Fig. 38 by comparing the relative intensities of the $\mathrm{O} 4$ and $\mathrm{O} 2+/ \mathrm{O} 3-$ phonons (i.e., $\mathrm{I}_{500} / \mathrm{I}_{340}$ ). Whereas "a-axis" grain growth exhibits an onset thickness for PLD-type YBCO films, as discussed above for the results in Fig. 36, "a-axis" grains seem to form in a thickness-independent manner for the ex situ-type of YBCO film, i.e., they persist in small quantity throughout the film as opposed to increasing in amount with increasing film thickness.

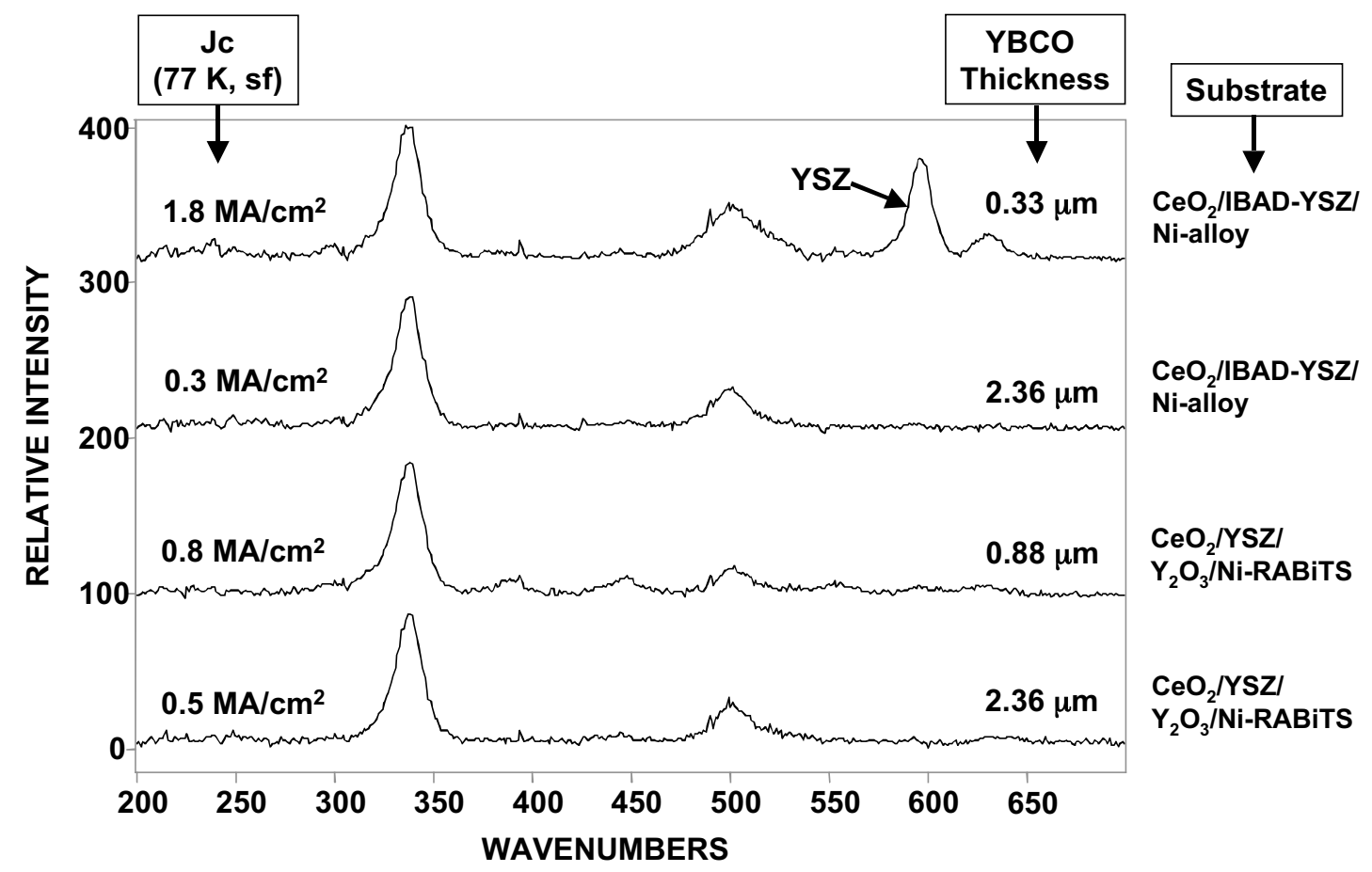

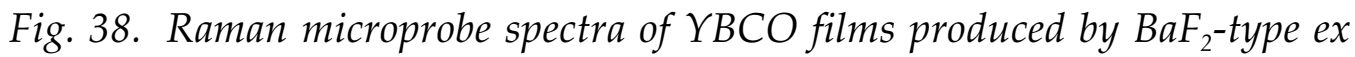
situ process. Information on type of substrate, film thickness, and measured $J_{c}$ is given for each film. IBAD substrates were produced at LANL; RABiTS substrates, at ORNL; films were deposited by $R$. Feenstra et al. at ORNL.

Figure 39 shows the Raman-generated texture maps for the four samples in Fig. 38, together with a graph of $\mathrm{J}_{\mathrm{c}}$ vs. YBCO thickness for various ex situ YBCOcoated conductor embodiments supplied by Feenstra et al. of ORNL. The quality/uniformity of the texture, which is reasonably good but not perfect, is comparable for all four samples. This finding seems to be relatively independent of substrate type, at least for the four samples we examined. From inspection of the data points in the inserted graph in Fig. 39, one can deduce that the dependence of $J_{c}$ on film thickness ( $t$ ) for ex situ YBCO generally conforms to a simple relationship of the form $\log \left(\mathrm{J}_{\mathrm{c}}\right) \propto-\log (\mathrm{t})$. 


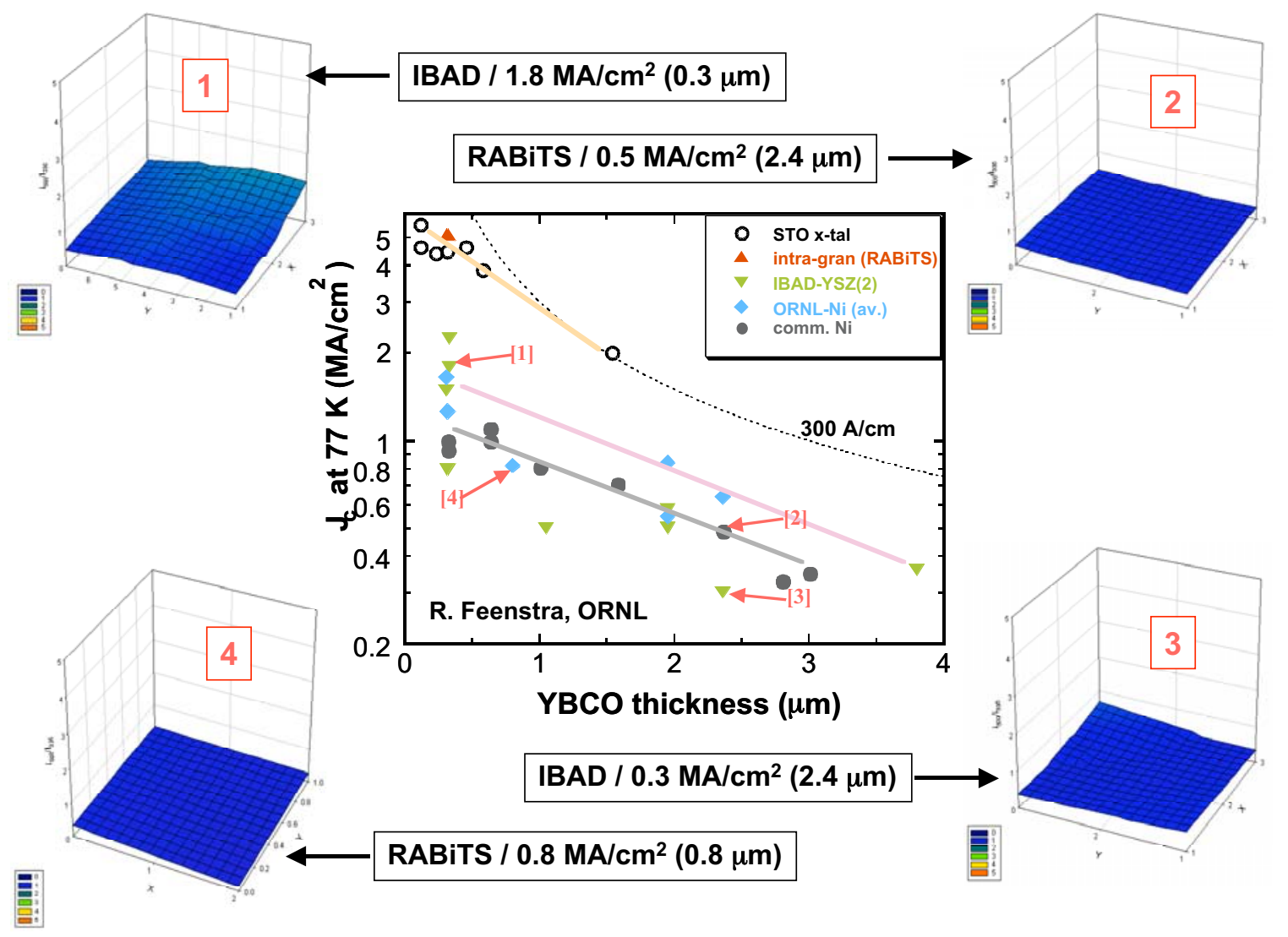

Fig. 39. Texture maps generated by Raman microprobe data for the four samples in Fig. 38. Graph shows $J_{c}$ vs. YBCO thickness for the four samples, together with values for other ex situ YBCO films on various types of substrates.

During the past year, we also used Raman microscopy to monitor phase evolution and texture quality in connection with the deposition of YBCO (by PLD) on rolled Ag and Ag alloy substrates. Several informative correlations were developed between film $J_{c}$ and process-related parameters. We found that adding a small percentage of $\mathrm{Cu}$ to the $\mathrm{Ag}$ improved $\mathrm{J}_{\mathrm{c}}$ values and that there appeared to be an optimum value for the $\mathrm{Cu}$ content. Figure 40a shows Raman spectra of four YBCO films on $\mathrm{Cu}-\mathrm{Ag}$ alloy substrates with a range of $\mathrm{Cu}$ contents. The $T_{c}$ and magnetization-derived driving current measured for each of these four YBCO films is plotted in Fig. $40 \mathrm{~b}$. The value of $\mathrm{I}_{500} / \mathrm{I}_{340}$ for each sample (also plotted Fig. 40b) reaches a minimum at $\mathrm{Ag}-0.2$ at.\% $\mathrm{Cu}$, which correlates with that specimen being the best performer in the group. Based on the criteria for texture quality discussed above (i.e., $\mathrm{I}_{500} / \mathrm{I}_{340} \rightarrow 0$ ), it is clear that the YBCO film on Ag-0.2 at.\% Cu exhibits the least evidence of tilted YBCO grains and no evidence of cation disorder $\left(\approx 580 \mathrm{~cm}^{-1}\right)$ or second phases. 

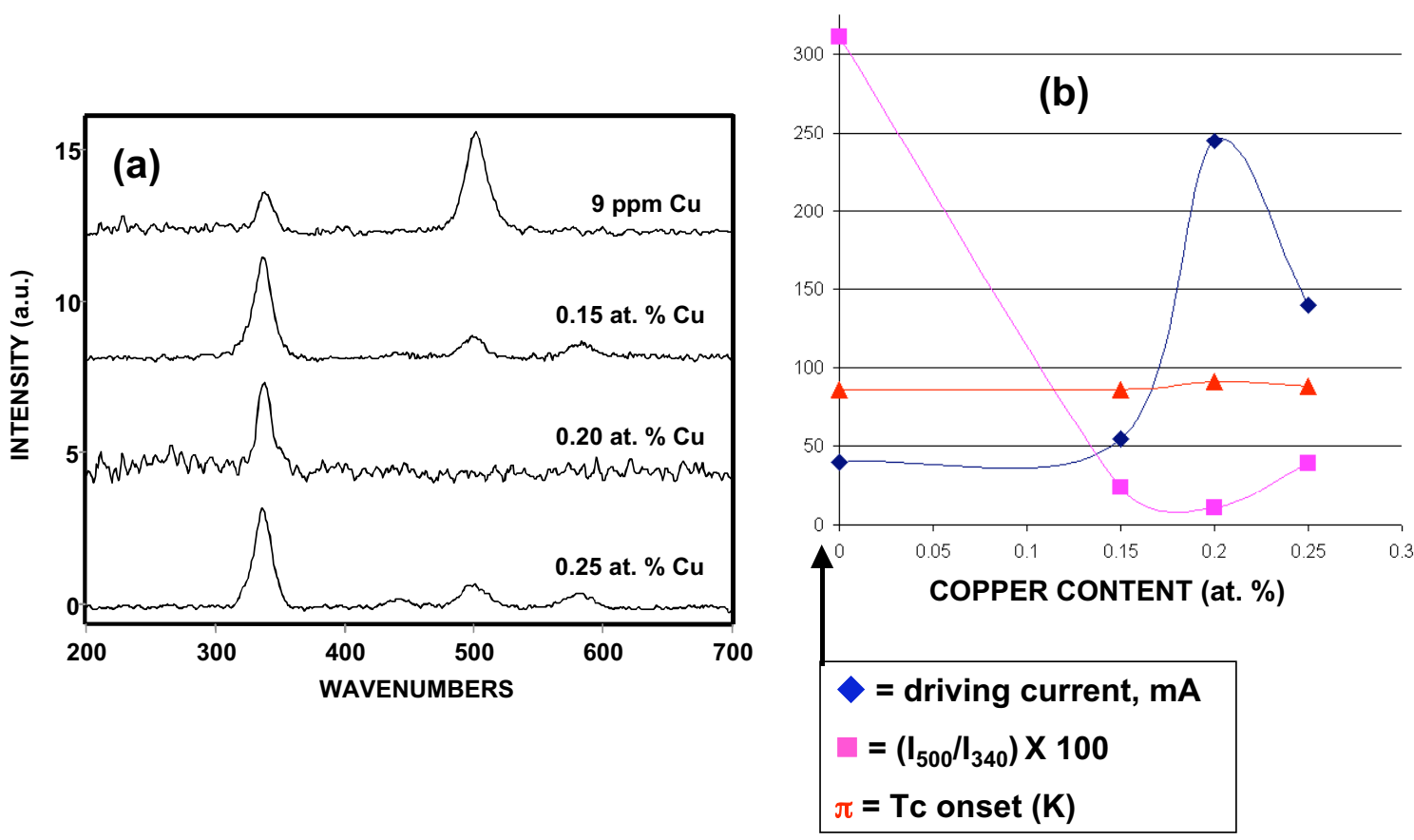

Fig. 40. (a) Raman microprobe spectra for PLD-type YBCO on roll-textured $A g-C u$ substrates with varying $\mathrm{Cu}$ content. (b) Combined plot of magnetization-based driving current (as a $J_{c}$ metric), $I_{500} / I_{340}$ value obtained from Raman spectra, and $T_{c}$ value for each $Y B C O$ film reported in (a) as a function of $\mathrm{Cu}$ content of substrate.

A study of the effect of laser pulse energy density on the performance of the deposited YBCO film also showed a correlation with texture quality. $I_{500} / I_{340}$ values obtained from the Raman spectra presented in Fig. 41a reached a minimum at the same energy density $\left(138 \mathrm{~mJ} / \mathrm{cm}^{2}\right)$ that yielded the best performing $\mathrm{YBCO} / \mathrm{Ag}-0.2$ at.\% $\mathrm{Cu}$ specimen, as shown in Fig. 41b. Also, the Raman spectrum of the sample deposited at a $130-\mathrm{mJ} / \mathrm{cm}^{2}$ pulse energy exhibited evidence of cation disorder $\left(\approx 580 \mathrm{~cm}^{-1}\right.$ in Fig. $\left.41 \mathrm{a}\right)$, which may be related to the observation that the driving current for this sample was lower than expected, based on the trend in driving current values evident in Fig. 41b. This observation adds to the mounting evidence in our Raman studies of YBCOcoated conductors indicating that cation disorder is detrimental to performance.

One of the objectives of our Raman microprobe investigations was to develop Raman-based methods for conducting on-line monitoring of long-length coated conductor manufacturing processes. A test study was conducted in collaboration with ChemIcon, Inc. (Pittsburgh, PA) to gauge focal-length requirements for sufficiently sensitive detection of YBCO phonons. Figure 42 summarizes results of Raman microprobe measurements that were performed on a $\mathrm{YBCO} / \mathrm{CeO}_{2} /$ Hastelloy- $\mathrm{C}$ specimen for a range of objective magnifications and working. 

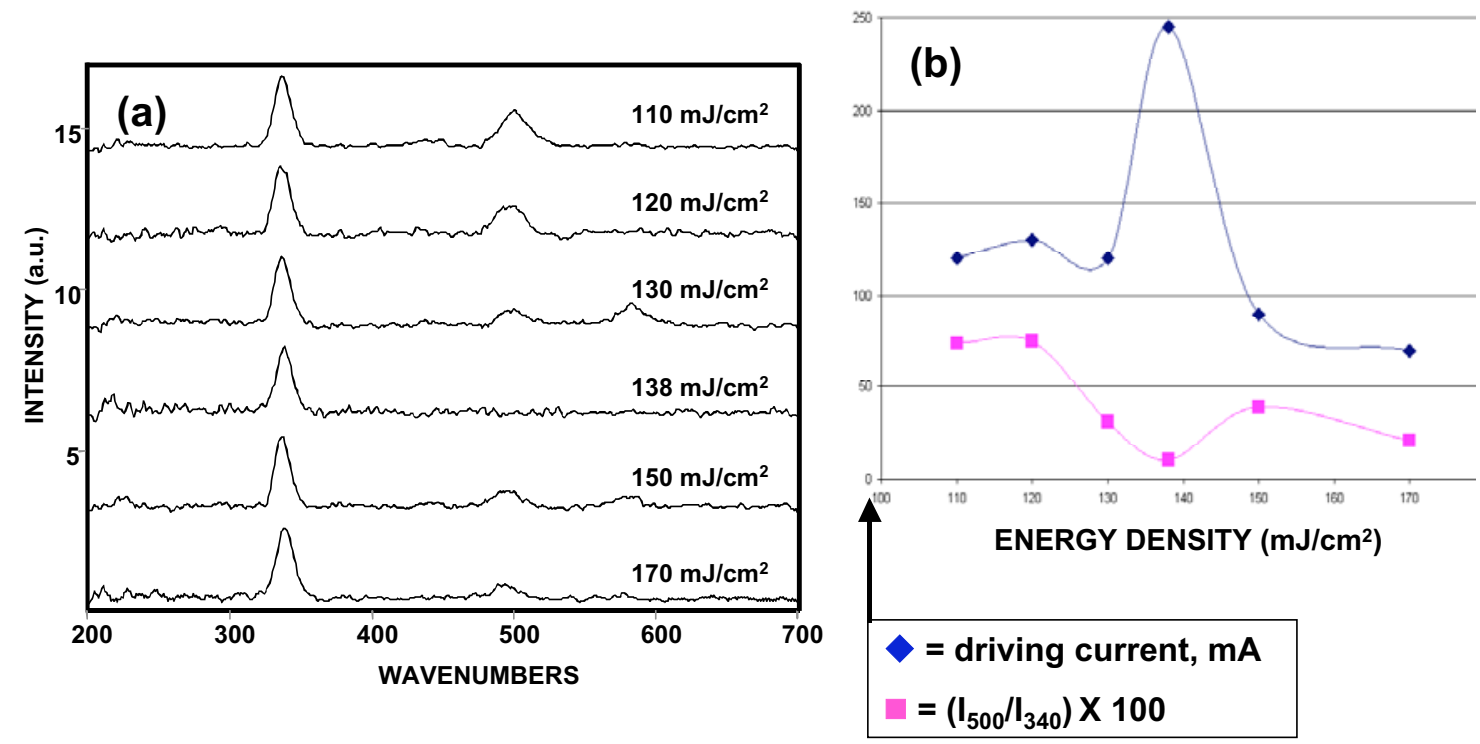

Fig. 41. (a) Raman microprobe spectra of PLD-type YBCO on Ag-0.2 at.\% Cu substrates deposited at differing laser pulse energy densities. (b) Combined plot of magnetization-based driving current (as a $J_{c}$ metric), and $I_{500} / I_{340}$ value obtained from the Raman spectra in (a) as a function of laser pulse energy density.

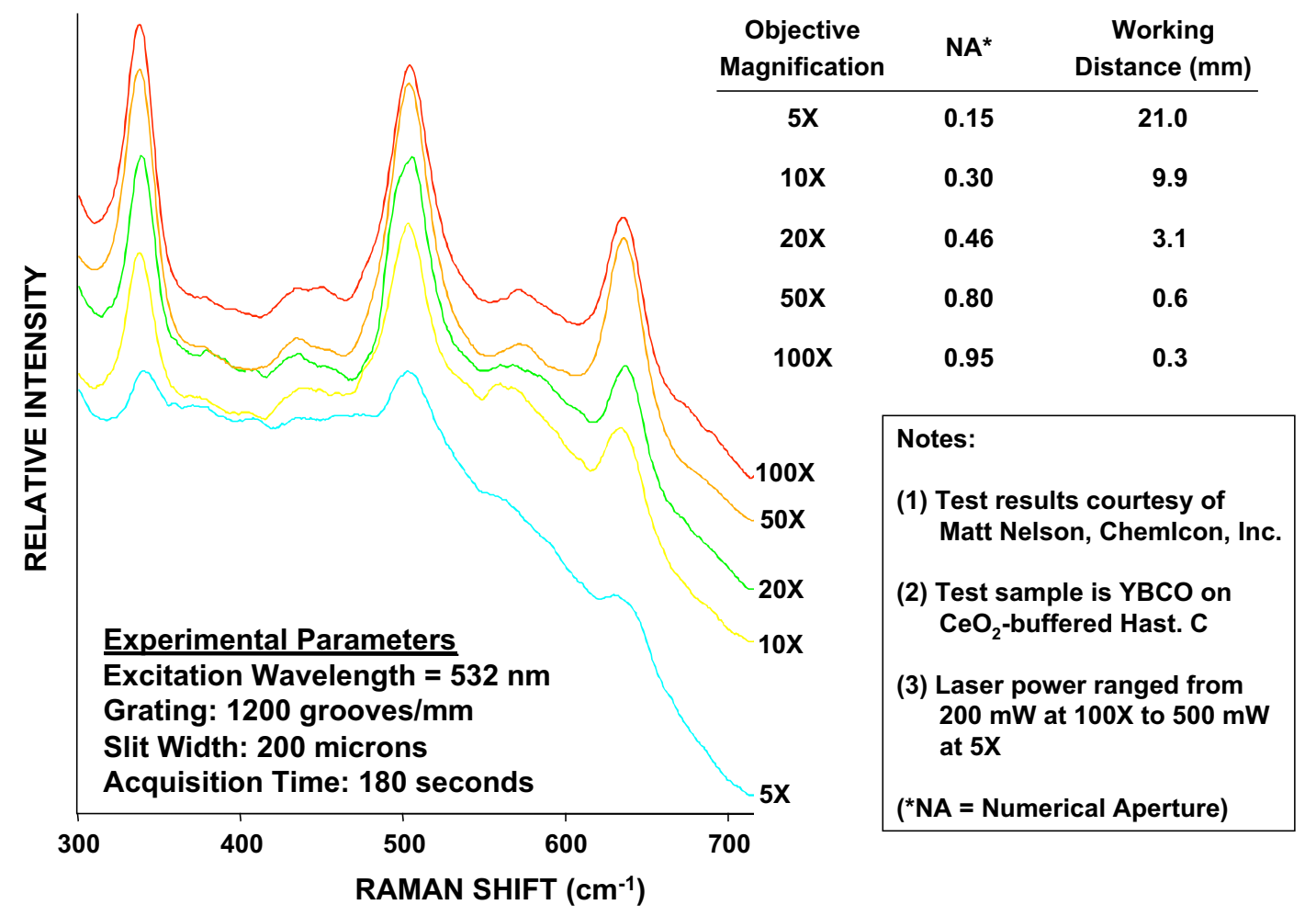

Fig. 42. Results of Raman microprobe measurements on YBCO-coatedconductor specimen as a function of objective magnification, numerical aperture (NA), and working distance. 
The results in Fig. 42 indicate that the O2+/O3- $\left(\approx 340 \mathrm{~cm}^{-1}\right)$ and $\mathrm{O} 4(\approx 500$ $\mathrm{cm}^{-1}$ ) modes of YBCO can be detected with useful sensitivity out to a working distance of $21 \mathrm{~mm}$. The Raman spectra in Fig. 42 were excited with a laser wavelength in the "green" portion of the visible spectrum, hence, the characteristic $\mathrm{BaCuO}_{2}$ mode $\left(\approx 640 \mathrm{~cm}^{-1}\right)$ is sufficiently enhanced that it is still noticeable out to the longest working distance $(21 \mathrm{~mm})$. Even the tetragonal YBCO mode at $\approx 450 \mathrm{~cm}^{-1}$ and the cation disorder mode at $\approx 580 \mathrm{~cm}^{-1}$ are discernable out to a working distance of nearly $10 \mathrm{~mm}$. These results provide encouraging evidence that Raman-based diagnostics could be used effectively to monitor phase evolution during YBCO-coated-conductor processing. At ambient temperature, the working distance could easily be as small as $0.5 \mathrm{~mm}$ without disturbing the surface of a moving tape. In elevated-temperature environments (even up to $800^{\circ} \mathrm{C}$ ), it should be possible to design/construct actively cooled optical bayonets that accommodate the taking of Raman spectra at working distances on the order of $20 \mathrm{~mm}$.

\subsection{4.b Basic Features of Raman Microscopy Relevant to YBCO}

Having demonstrated that Raman microscopy is a powerful, comprehensive characterization tool for examining MBCO-coated conductors, we provide here a rudimentary discussion of the Raman active phonon properties of the $\mathrm{MBCO}$ crystal structure for $\mathrm{x}=0$ (orthorhombic $\mathrm{MBCO}$ ) and $\mathrm{x}=$ 1 (tetragonal $\mathrm{MBCO}$ ). We couple that discussion with a description of how the observational features of these phonons are influenced by the epitaxial (singlecrystal-like) nature of $\mathrm{MBCO}$ films on textured substrates. We then describe the results of a detailed Raman microprobe study of a 1.25-m-long YBCO coated conductor specimen produced by Dominic Lee at ORNL.

From factor group analysis one determines that both the orthorhombic and tetragonal MBCO structures (referred to herein as $\mathrm{MBCO} / \mathrm{O}$ and $\mathrm{MBCO} / \mathrm{T}$, respectively) have fifteen Raman active vibrational degrees of freedom [7]. For $\mathrm{MBCO} / \mathrm{O}$, the motional vector of five of these phonons is along the $\mathrm{c}$-axis direction, whereas the motional vector for the other ten phonons is in the $\mathbf{a}, \mathbf{b}$ planes. For $\mathrm{MBCO} / \mathrm{T}$, there are, again, five nondegenerate phonons, with motional vectors along the c-axis and five doubly degenerate phonons with motional vectors in the $\mathbf{a}, \mathbf{b}$ planes. The only phonons regularly detected for $\mathrm{MBCO}$ are the ones with motional vectors along the c-axis. One speculation about why this is so is based on the notion that MBCO exhibits characteristics of a ceramic-like material in the c-axis direction, and those of a pseudometal in the $\mathbf{a}, \mathbf{b}$ planes. Because metals are notoriously poor Raman scatterers when a visible wavelength excitation source (invariably a laser) is used to excite the Raman scattering, it is not surprising that the $\mathbf{a}, \mathbf{b}$-plane phonons (mostly involving 
vibrations in the copper-oxygen planes of $\mathrm{MBCO}$ ) fail to appear in visiblewavelength-excited Raman spectra.

The motional vectors for the five c-axis phonons of the MBCO structure, which turn out to be identical for both $\mathrm{MBCO} / \mathrm{O}$ and $\mathrm{MBCO} / \mathrm{T}$, are shown in Fig. 43, together with typical values for the phonon frequencies of the two MBCO crystal types. Each of these phonons has its own unique set of polarizability tensor elements, and these sets produce various scattering intensities, depending on the orientation of the $\mathrm{MBCO}$ crystal with respect to the direction of propagation of the incident laser $(\lambda)$ and the direction of observation $(I)$ of the Raman scattering [8]. Typically, when a Raman microprobe is used to examine an MBCO coated conductor specimen, the excitation/detection configuration is such that the introduction of the laser and the observation of Raman scattering are both performed perpendicular to the plane of the substrate (i.e., by employing a common optical element) and, hence, parallel to the c-axis of the $\mathrm{MBCO}$ film. The observed intensities of the $\mathrm{MBCO} / \mathrm{O}$ and $\mathrm{MBCO} / \mathrm{T}$ phonons for this case are illustrated in Fig. 44 by the two spectra marked with the designation $\lambda / /$ c \& $I / /$ c. These spectra were obtained by using oriented bulk specimens of $\mathrm{YBCO} / \mathrm{O}$ and $\mathrm{YBCO} / \mathrm{T}$ produced by zone-melting methods.

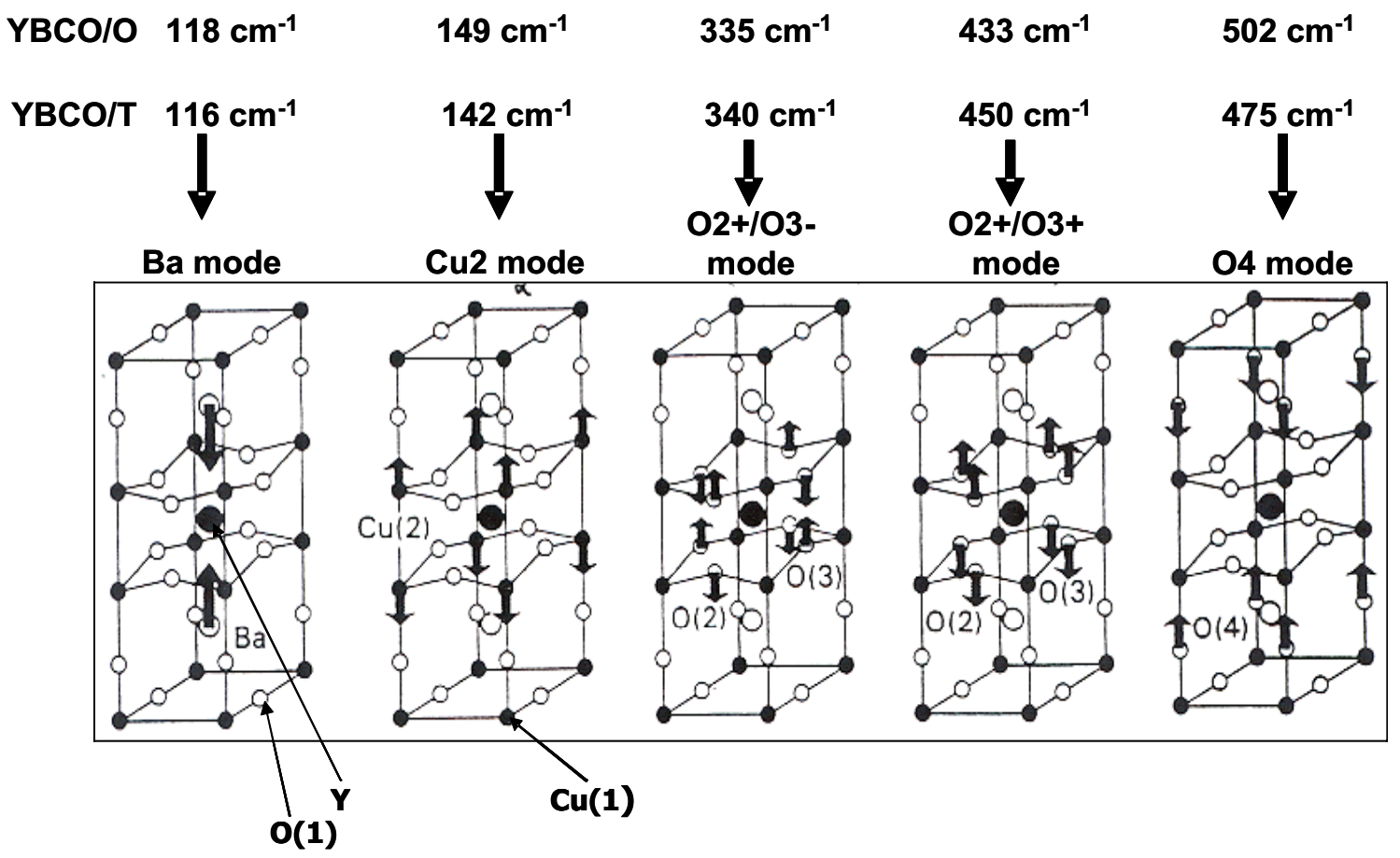

Fig. 43. Atom motion vectors for the five Raman-active c-axis phonons of orthorhombic and tetragonal MBCO (MBCO/O and MBCO/T, respectively), together with typical frequency values for each $M B C O$ crystal type. 


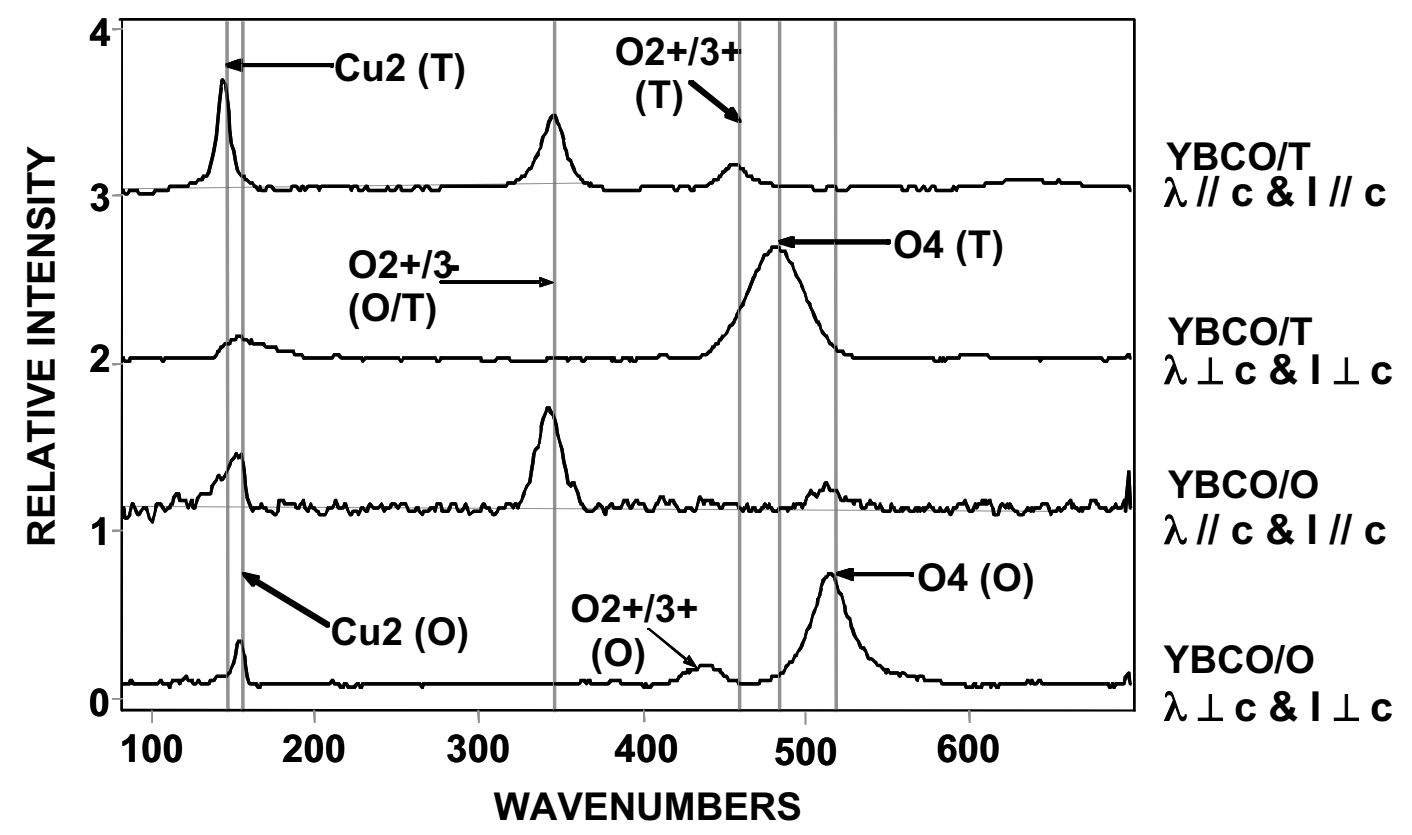

Fig. 44. Raman spectra of bulk, textured (melt-processed) samples of MBCO/O and $M B C O / T$ for two orientations of incident excitation laser $(\lambda)$ and observation direction (I).

A strikingly different phonon intensity pattern is obtained when both $\lambda$ and $I$ are perpendicular to the c-axis. In Fig. 44, we also show the corresponding spectra for this case (designated as $\lambda \perp \mathbf{c} \& I \perp \mathbf{c}$ ) because it comes into play when the MBCO grains tip out of the plane of the substrate and/or when "a-axis" grain growth occurs. Note in Fig. 44 that, for perfectly epitaxial $\mathrm{MBCO} / \mathrm{O}$ in the $\lambda$ / / c \& I / / c configuration, one should observe the O2+/O3- and Cu2 modes but not the $\mathrm{O} 4$ mode. Conversely, in the $\lambda \perp \mathbf{c} \& I \perp \mathbf{c}$ configuration, one should observe the $\mathrm{O} 4$ and $\mathrm{Cu} 2$ modes but not the $\mathrm{O} 2+/ \mathrm{O} 3$ - mode. In the case of a specimen composed of a mixture of epitaxial, tilted, and/or a-axis $\mathrm{MBCO} / \mathrm{O}$ grains, one is likely to observe Raman spectra that are a mix of the ones shown in Fig. 44. Furthermore, if $\mathrm{MBCO} / \mathrm{T}$ is present alone or in combination with $\mathrm{MBCO} / \mathrm{O}$, one is likely to observe a spectrum composed of a mix of two or more of the four spectra shown in Fig. 44.

Using the results in Fig. 44 as a basis, we can determine many important characteristics of $\mathrm{MBCO}$ coated conductor films from their Raman spectra. For example, we can gauge the relative amounts of epitaxial versus tilted/a-axis grains from the relative intensities of the $\mathrm{O} 2+/ \mathrm{O} 3-$ and $\mathrm{O} 4$ modes, distinguish $\mathrm{MBCO} / \mathrm{O}$ from $\mathrm{MBCO} / \mathrm{T}$ by using the frequencies of the $\mathrm{O} 4, \mathrm{Cu} 2$, and $\mathrm{O} 2+/ \mathrm{O} 3+$ phonons, and estimate the extent of oxygenation of $\mathrm{MBCO}$ (i.e., the value of $\mathrm{x}$ ) from the frequency of the $\mathrm{O} 4$ mode. Coupling this capability with our 
previously reported success in detecting many of the relevant secondary nonsuperconducting phases that occur during precursor heat treatment to form $\mathrm{MBCO}$ films (e.g., $\mathrm{CuO}, \mathrm{BaF}_{2}, \mathrm{BaCeO}_{3}$, and barium cuprates), it becomes demonstrably clear that Raman microscopy is a globally useful methodology for interrogating $\mathrm{MBCO}$ coated conductors. Furthermore, to make the technique applicable to long-length conductors, we have constructed a reel-to-reel attachment for our existing Raman microprobe (see Fig. 45) that allows us to examine tapes up to $12 \mathrm{~m}$ long. In the next section, we describe the application of this methodology to specific meter-length coated conductor specimens.

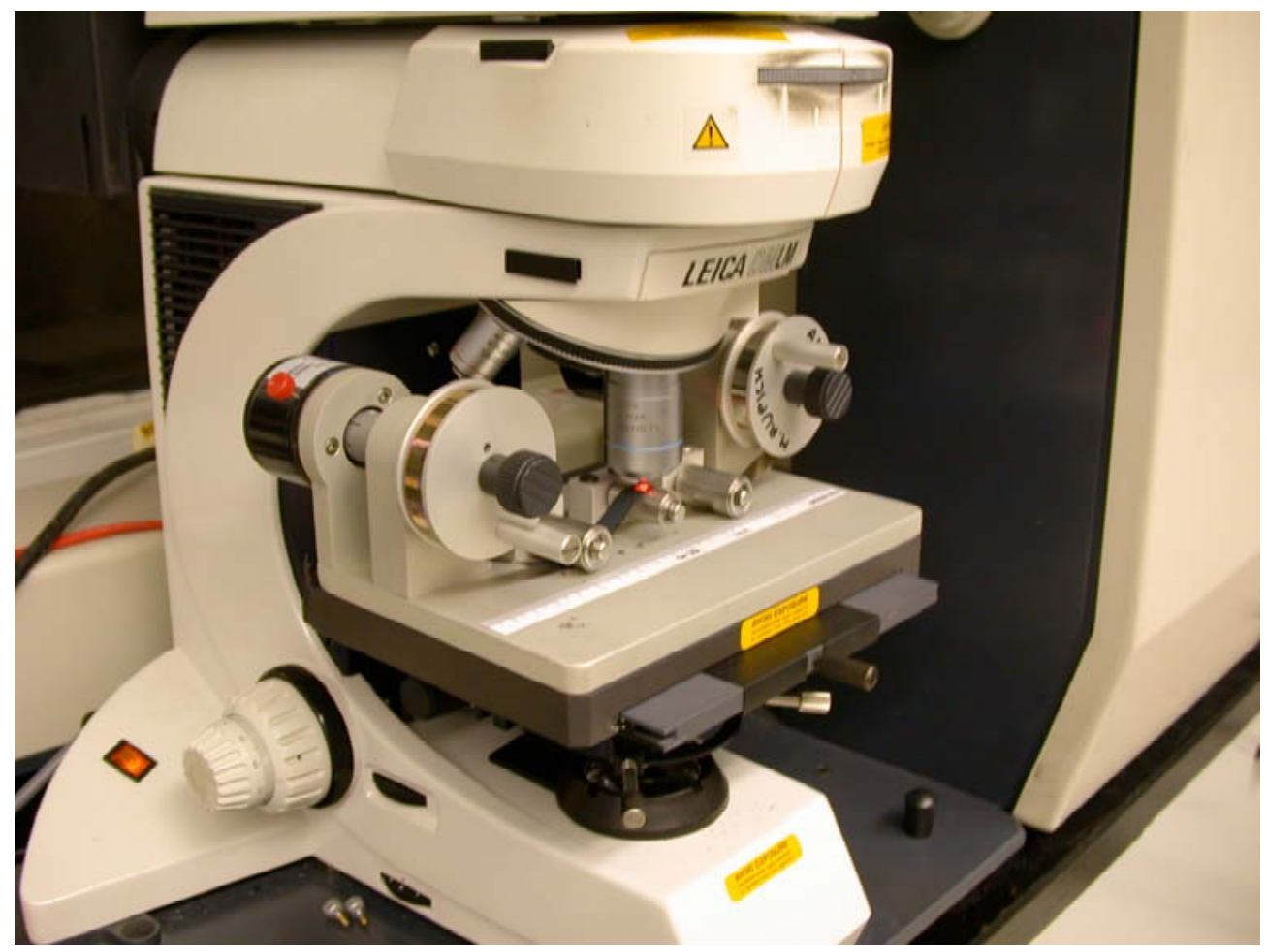

Fig. 45. Picture of reel-to-reel $(R 2 R)$ device used to feed and control longlength coated conductor tape specimens during Raman microscopy examinations. $R 2 R$ device is shown in position on microscope stage of Raman microprobe.

\subsection{4.c Raman Microscopy for Examining YBCO Precursor Evolution}

Reel-to-reel (R2R) Raman microscopy is now being performed routinely in our laboratory. Although the technique provides substantive information when employed to examine fully processed tapes, we have also found that it is especially useful for characterizing phase evolution in tapes that have been processed to obtain a gradation of transformed precursor states. Specifically, Dominic Lee at ORNL has developed a method for creating such tapes, which we 
call time-gradient-processed (TGP) tapes. In this method, a precursor-coated substrate is reeled into/through a preheated furnace at a controlled rate such that each increment of the precursor spends a different amount of time at the selected precursor transformation temperature. The tape feed rate is chosen such that the leading edge of the precursor is overprocessed, the trailing edge is essentially unprocessed, and an entire spectrum of phase conversion states exists in between. When the leading edge of the coated section reaches the end of the hot zone, the tape is rapidly reeled back out of the furnace to quench the existing phase states.

During FY 2003, we examined two such TGP tapes produced by Dominic Lee. Both were $\mathrm{YBCO} / \mathrm{CeO}_{2}(150 \mathrm{~nm}) / \mathrm{YSZ}(200 \mathrm{~nm}) / \mathrm{Y}_{2} \mathrm{O}_{3}(15 \mathrm{~nm}) / \mathrm{Ni}(1$ $\mu \mathrm{m}) / \mathrm{Ni}-\mathrm{W}(50 \mu \mathrm{m})$ embodiments that consisted of $\approx 1.25$ meters of electron-beamdeposited $\mathrm{Y}-\mathrm{BaF}_{2}-\mathrm{Cu}$ precursor (with sufficient leader tape attached to each end to allow reeling). The specimens were coated to produce two thicknesses of $\mathrm{YBCO}, 280 \mathrm{~nm}$ in one case and $1000 \mathrm{~nm}$ in the second case. Inasmuch as the results obtained by Raman microscopy examination of these two tapes were the same in nearly all respects, we limit the discussion here to the findings for the $280 \mathrm{~nm}$ YBCO tape.

A sketch of the time/temperature/distance relationship for the 280-nm YBCO tape is shown in Fig. 46. The precursor transformation temperature used for this sample was $740^{\circ} \mathrm{C}$. The consecutive increments of the precursor spent times ranging from "zero" to $150 \mathrm{~min}$ at this temperature. In addition, there were increments of precursor near the trailing end of the coated section (the ramp section) that experienced peak temperatures ranging from 600 to $740^{\circ} \mathrm{C}$ before the quench. In the Raman microscopy examination of this tape, we used a partially defocused excitation laser ( $633 \mathrm{~nm}$ wavelength) to probe two spots $(\approx 6$ $\mu \mathrm{m}$ in dia.) at each distance along the tape. The two spots were chosen to be one quarter of the way in from the top edge (as depicted in Fig. 46) and three quarters of the way in, respectively. This was done to look for evidence of transverse variations in the phase composition along the length of the precursor coating. However, because a discernable difference in the two spectra was seen in very few instances, we focus here on the end-to-end variations by using the averaged value of the two Raman spectra recorded at each probed lengthwise location. The following paragraphs present a segment-by-segment synopsis that starts at the trailing end of the precursor zone, highlights the key findings and implications of the Raman results, and points out the instances where these results reinforce and transcend the findings of an R2R XRD analysis of the same tape performed at ORNL. 


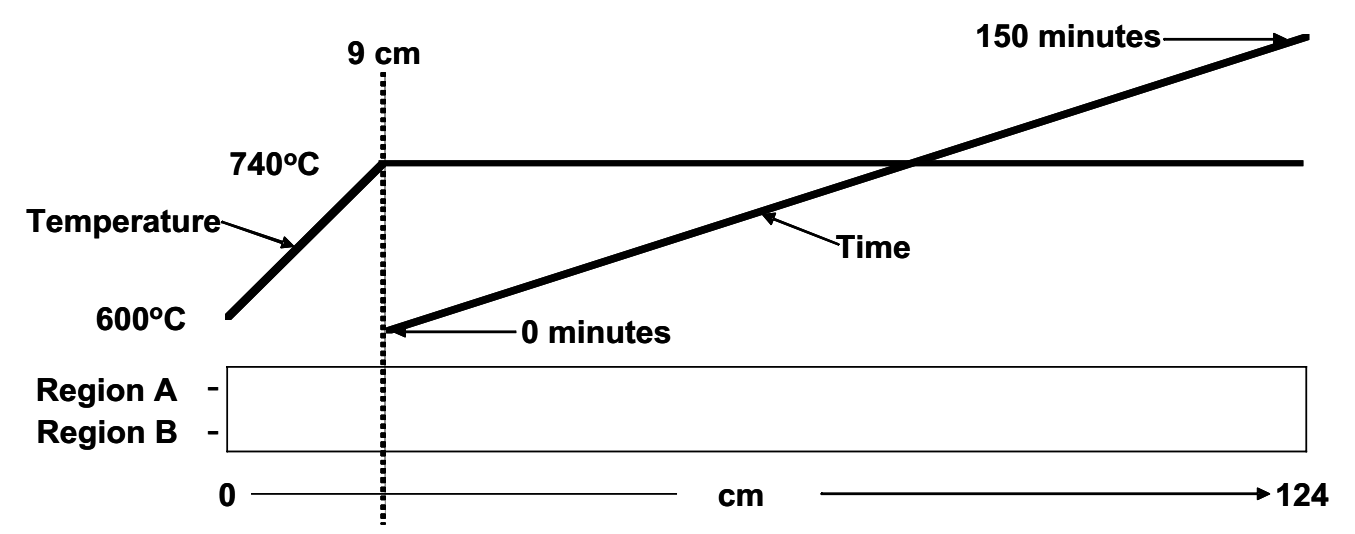

Fig 46. Schematic diagram illustrating time and temperature profiles used by D. Lee at ORNL to produce time-gradient-processed 280-nm-thick YBCO film on 1.25 m metal substrate.

The Ramp Zone (650 to $740^{\circ} \mathrm{C}$ ) The Raman spectra of increments of the precursor in this zone (see Fig. 47) exhibit two broad diffuse band envelopes, one between 100 and $200 \mathrm{~cm}^{-1}$ and another between 500 and $700 \mathrm{~cm}^{-1}$, indicating the dominant presence of an amorphous mush from which $\mathrm{CuO}\left(\approx 300 \mathrm{~cm}^{-1}\right)$ appears as the first crystalline phase. $\mathrm{BaF}_{2}$, the cubic form of which exhibits a characteristic phonon at $242 \mathrm{~cm}^{-1}$, is not detected in this region ( 3 to $8 \mathrm{~cm}$ ) by Raman microscopy, and the ORNL XRD results likewise show little evidence of cubic $\mathrm{BaF}_{2}$ in this region. By the time the precursor has reached $740^{\circ} \mathrm{C}$, the $\mathrm{CuO}$ phonon dominates the spectrum, but other sharp modes begin to appear as shown in Fig. 48, where we present a comparison of the 3-cm increment $\left(650^{\circ} \mathrm{C}\right.$ maximum temperature) and the $9-\mathrm{cm}$ increment (initial minute at $740^{\circ} \mathrm{C}$ ) to provide a beginning-to-end perspective of the aggregate phase transformation that takes place in the ramp segment.

Overview of Phase Evolution on the $740^{\circ} \mathrm{C}$ Plateau Figure 49 presents an overview of phase transformation along the $740^{\circ} \mathrm{C}$ plateau. On the right-hand side of this figure, we correlate each spectrum with the elapsed time at $740^{\circ} \mathrm{C}$ to provide a global view of the overall reaction kinetics. In just a few minutes at $740^{\circ} \mathrm{C}$, the broad bands associated with the amorphous mush are completely gone, and several new crystalline phases (in addition to $\mathrm{CuO}$ ) are clearly evident. In just a little over ten min, phonons attributable to $\mathrm{BaF}_{2}$ and $\mathrm{BaCeO}_{3}$ are apparent, YBCO is starting to form, and other bands are present in the spectra, which we will subsequently discuss and assign. In a little over $30 \mathrm{~min}$ the spectra are dominated by $\mathrm{YBCO} / \mathrm{T}$ phonons; in fact, the last vestiges of $\mathrm{CuO}$ appear at the 34-min point. It is noteworthy that the ORNL XRD scans of this region detected the in-growth of $\mathrm{BaF}_{2}$ and $\mathrm{BaCeO}_{3}$ and the formation of $\mathrm{YBCO}$ (not specifically the " $\mathrm{T}$ " form) but not the $\mathrm{CuO}$ or other new phases. 


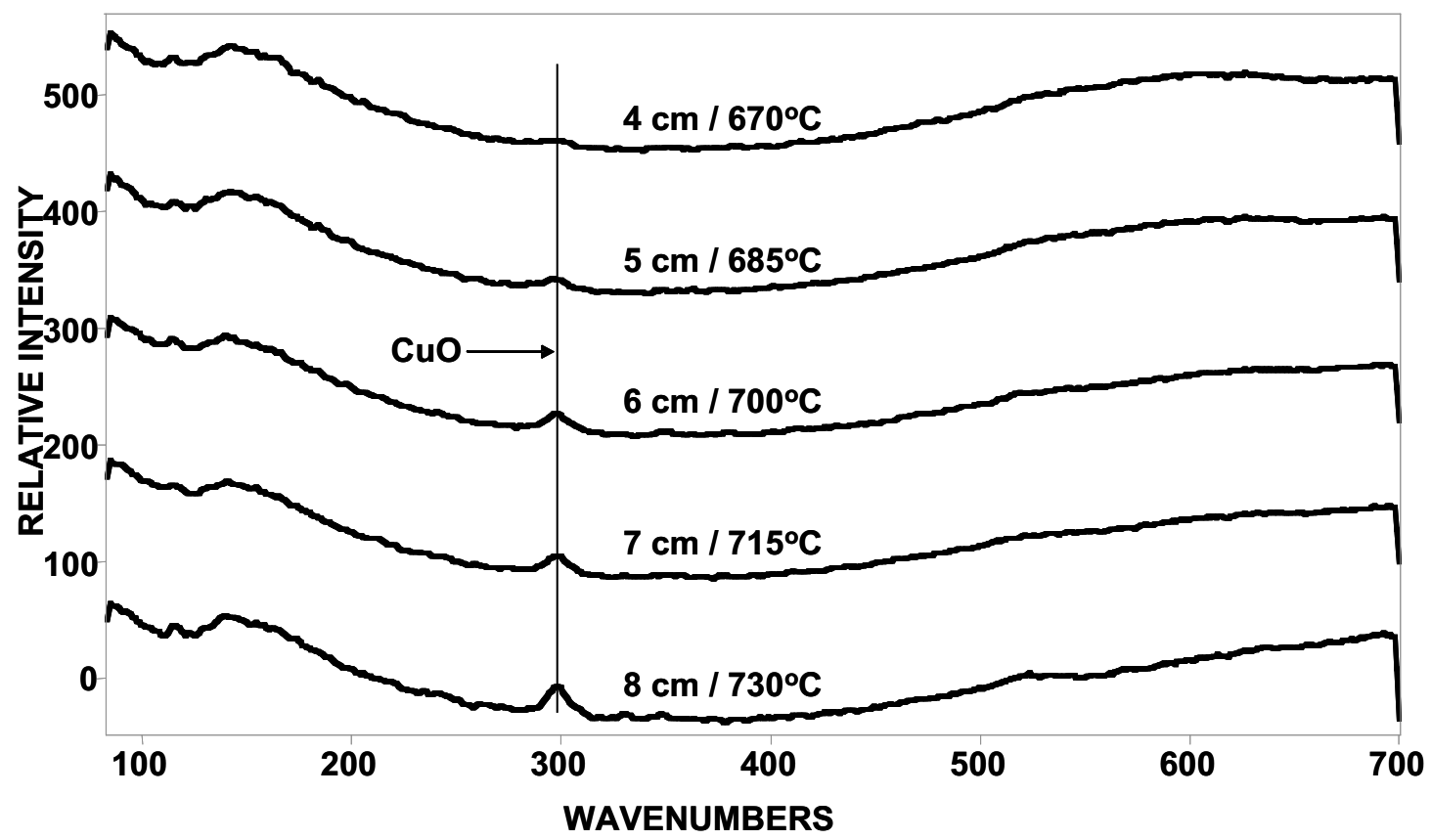

Fig. 47 Raman spectra of $\mathrm{Y}-\mathrm{BaF}_{2}-\mathrm{Cu}$ precursor at five increments in ramp zone of 1.25-m time-gradient-processed tape received from ORNL. Indicated temperature is the maximum temperature reached before quench at that location.

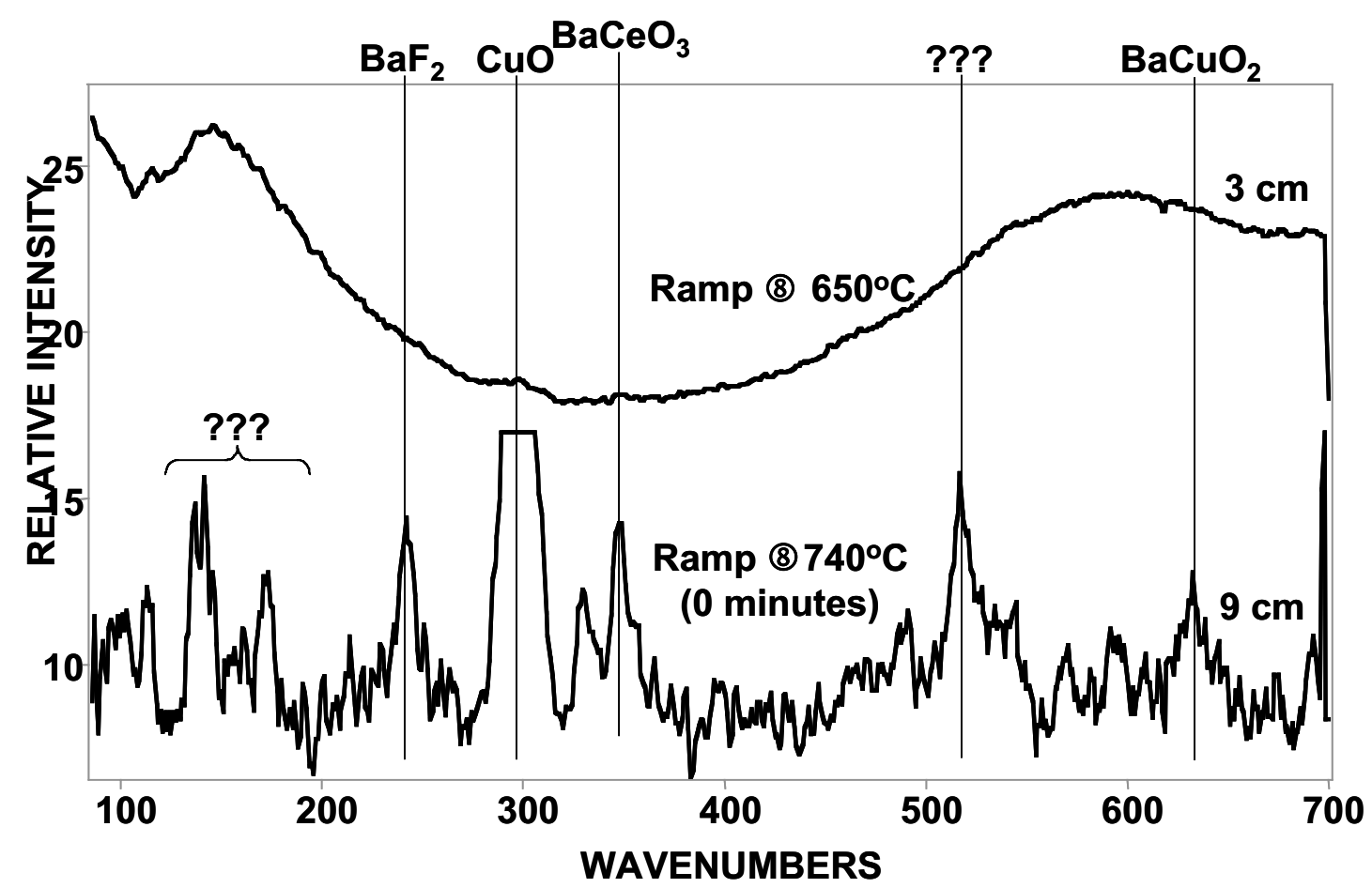

Fig. 48 Raman spectra recorded at $650^{\circ} \mathrm{C}$ ramp increment and at increment where precursor just reached transformation temperature $\left(740^{\circ} \mathrm{C}\right)$ before quench. Note onset of crystallization in latter case (lower spectrum). 


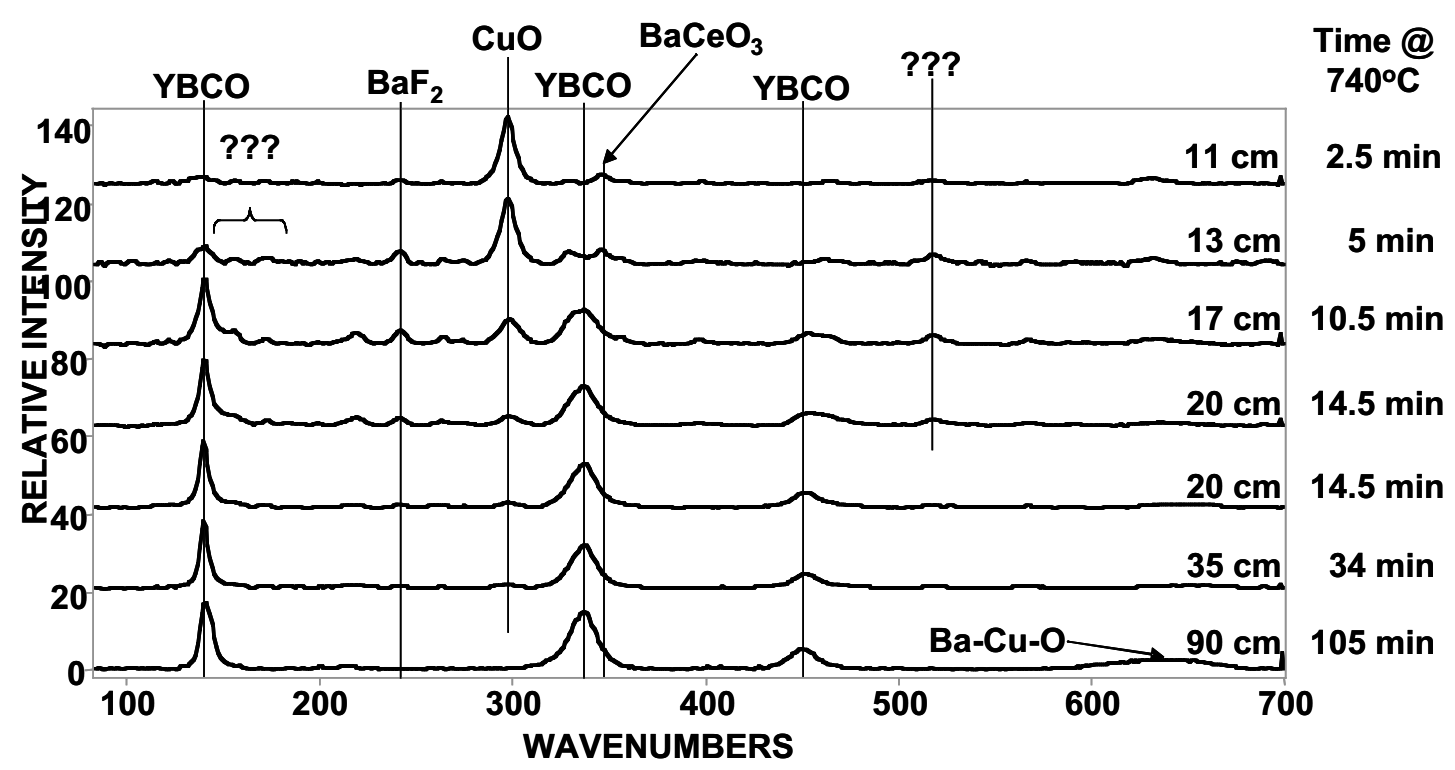

Fig. 49 Raman spectra of increments along portion of 1.25-m time-gradientprocessed $Y B C O$ tape that reached treatment temperature of $740^{\circ} \mathrm{C}$. Time each increment spent at $740^{\circ} \mathrm{C}$ before quench is indicated along right side of plot.

New Phases in the First 20 Min at $740^{\circ} \mathrm{C}$ A series of bands that begins to emerge in the first few minutes at $740^{\circ} \mathrm{C}$ and persists through the first $20 \mathrm{~min}$ exhibits a compelling correlation with the phonon pattern of the $\mathrm{Y}_{2} \mathrm{Cu}_{2} \mathrm{O}_{5}$ phase (referred to hereinafter as 202). This correlation can be seen in Fig. 50, where we plot the precursor spectra for $\approx 0$ and $\approx 16$ min of treatment at $740^{\circ} \mathrm{C}$, together with the spectrum of pure 202 powder, which was recorded under the same instrument conditions used to examine the $1.25-\mathrm{m}$ tape. The correlation at $0 \mathrm{~min}$ is clearer than that at $16 \mathrm{~min}$, where $\mathrm{YBCO}$ and $\mathrm{BaF}_{2}$ modes tend to mask some of the 202 modes. In addition, modes in the range $590-650 \mathrm{~cm}^{-1}$ are attributed to barium cuprates (Ba-Cu-O) [7]. These results imply that the 202 and $\mathrm{Ba}-\mathrm{Cu}-\mathrm{O}$ phases (along with $\mathrm{CuO}$ ) are intermediates to $\mathrm{YBCO}$ formation. Furthermore, none of these phases was conclusively identified during the ORNL XRD examinations. Figure 51 is included to show that these phases persist through the early stages of YBCO formation.

The Heartland of YBCO Formation and the "Sweet Spot" Figure 52 presents Raman spectra that cover the time range at $740^{\circ} \mathrm{C}$, extending from the final appearance of modes due to intermediates (i.e., $\mathrm{CuO}, 202$, and $\mathrm{Ba}-\mathrm{Cu}-\mathrm{O}$ ), through the region of optimum $\mathrm{YBCO} / \mathrm{T}$ presence, to the onset of an overprocessed state, where $\mathrm{Ba}-\mathrm{Cu}-\mathrm{O}$ modes reappear. We believe these results define a "sweet spot" in the time-at $-740^{\circ} \mathrm{C}$ progression where the film is composed of nearly phase-pure $\mathrm{YBCO} / \mathrm{T}$. 


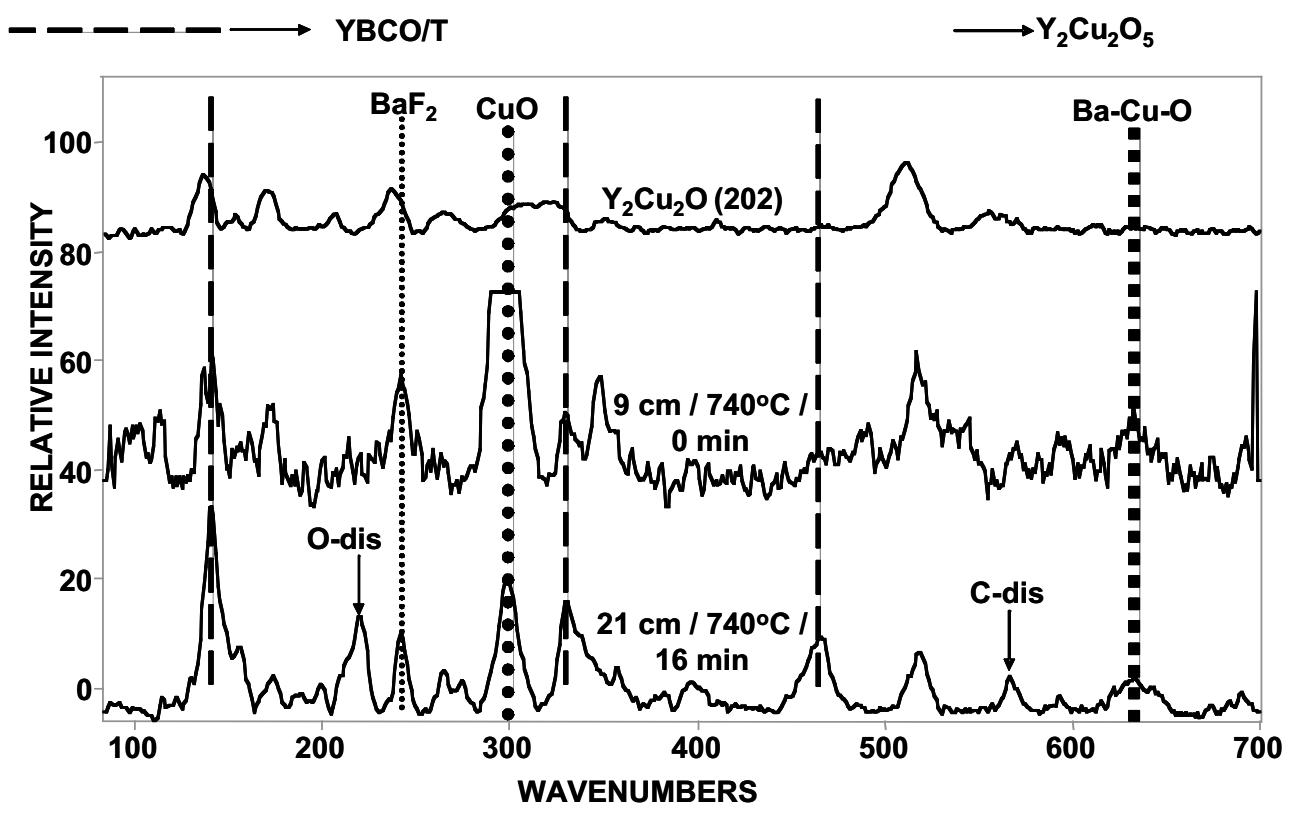

Fig. 50 Plot correlating Raman bands for pure $\mathrm{Y}_{2} \mathrm{Cu}_{2} \mathrm{O}_{5}$ powder (top spectrum) with bands seen early in $Y B C O$ precursor transformation during time-gradientprocessing of YBCO tape. Middle spectrum is for 9-cm increment that just reached $740^{\circ} \mathrm{C}$ before quench; lower spectrum for 16-cm increment that spent $\approx 16$ min at $740^{\circ} \mathrm{C}$ before quench. "O-dis" and "C-dis" are bands attributed to oxygen-disorder-and cation-disorder-induced phonons of YBCO [9].

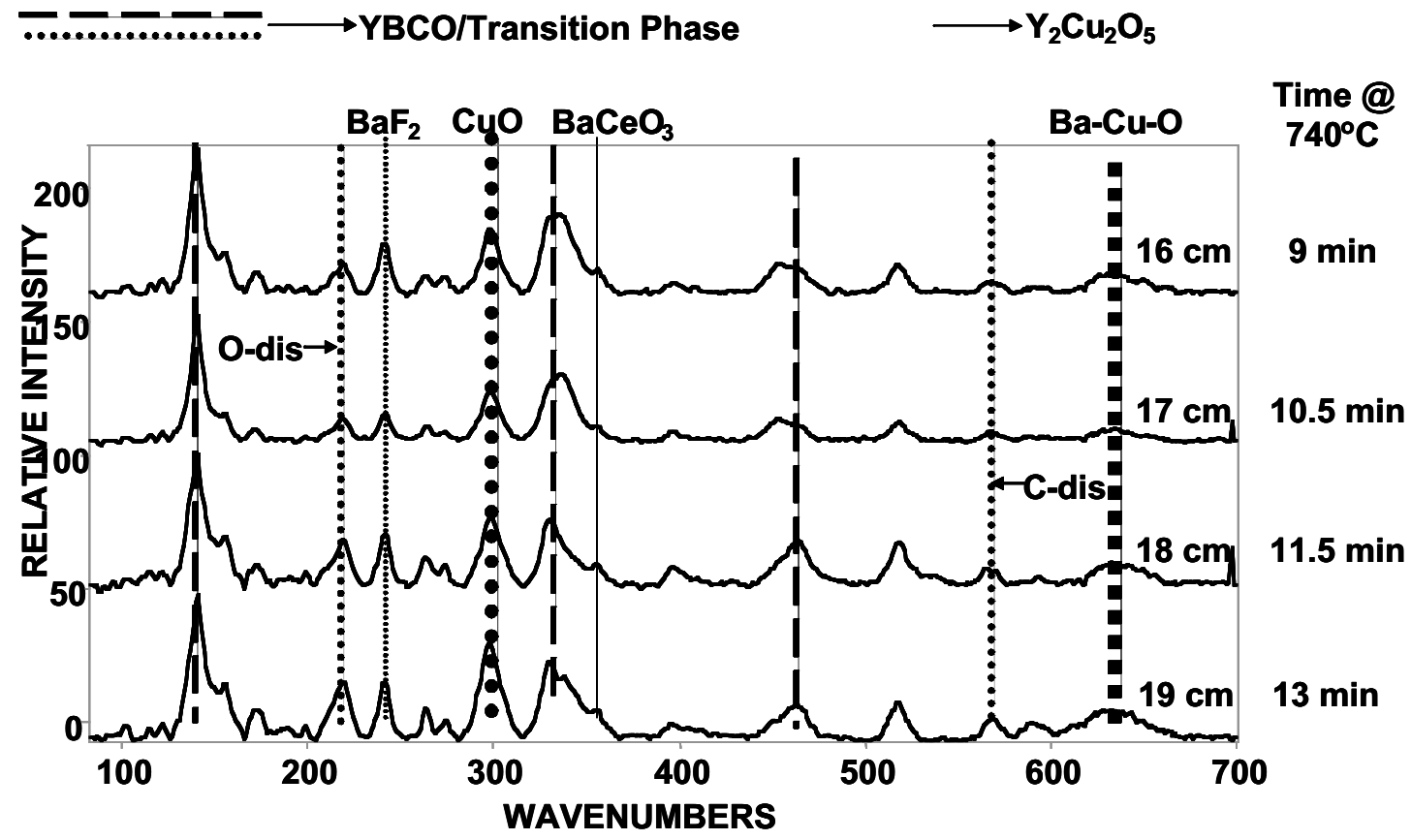

Fig. 51 Raman spectra of increments in midportion of phase transition region, showing persistence of $\mathrm{Y}_{2} \mathrm{Cu}_{2} \mathrm{O}_{5}$ and $\mathrm{CuO}$ phonons in early stages of $\mathrm{YBCO}$ formation along 1.25-m YBCO time-gradient-processed tape. 
Figure 52 shows that the "sweet spot" during processing occurs in the range from 50 to $70 \mathrm{~min}$ at $740^{\circ} \mathrm{C}$. In the blowup of the 40-79-min region, shown in Fig. 53 , we see little evidence of any phonons other than the three characteristic ones due to $\mathrm{YBCO} / \mathrm{T}$ but do note the onset of the well-studied YBCO cation disorder mode $\left(\approx 580 \mathrm{~cm}^{-1}[9]\right)$ in the 79-min spectrum. The "sweet spot" observed for the 1000 -nm YBCO tape specimen produced by Dominic Lee corresponds to $\approx 220$ $230 \mathrm{~min}$ at $740^{\circ} \mathrm{C}$ (roughly four times that for the 280-nm YBCO tape specimen), illustrating the same approximately linear relationship between processing time and YBCO film thickness that was deduced from the ORNL XRD measurements.

By recording dozens of individual Raman spectra along the length of the ORNL TGP tapes and consolidating them into three-dimensional histograms, the phase evolution can be graphically portrayed to reveal many of the salient features of the precursor conversion process. Figure 54 shows a typical histogram that portrays the Raman band frequency (in wavenumbers) and the relative band intensity as a function of distance (and concomitantly, time) with respect to the tape origin. This particular histogram covers the major portion of the $\approx 300 \mathrm{~nm}$ precursor tape that experienced the soak temperature $\left(740^{\circ} \mathrm{C}\right)$ for some period of time.

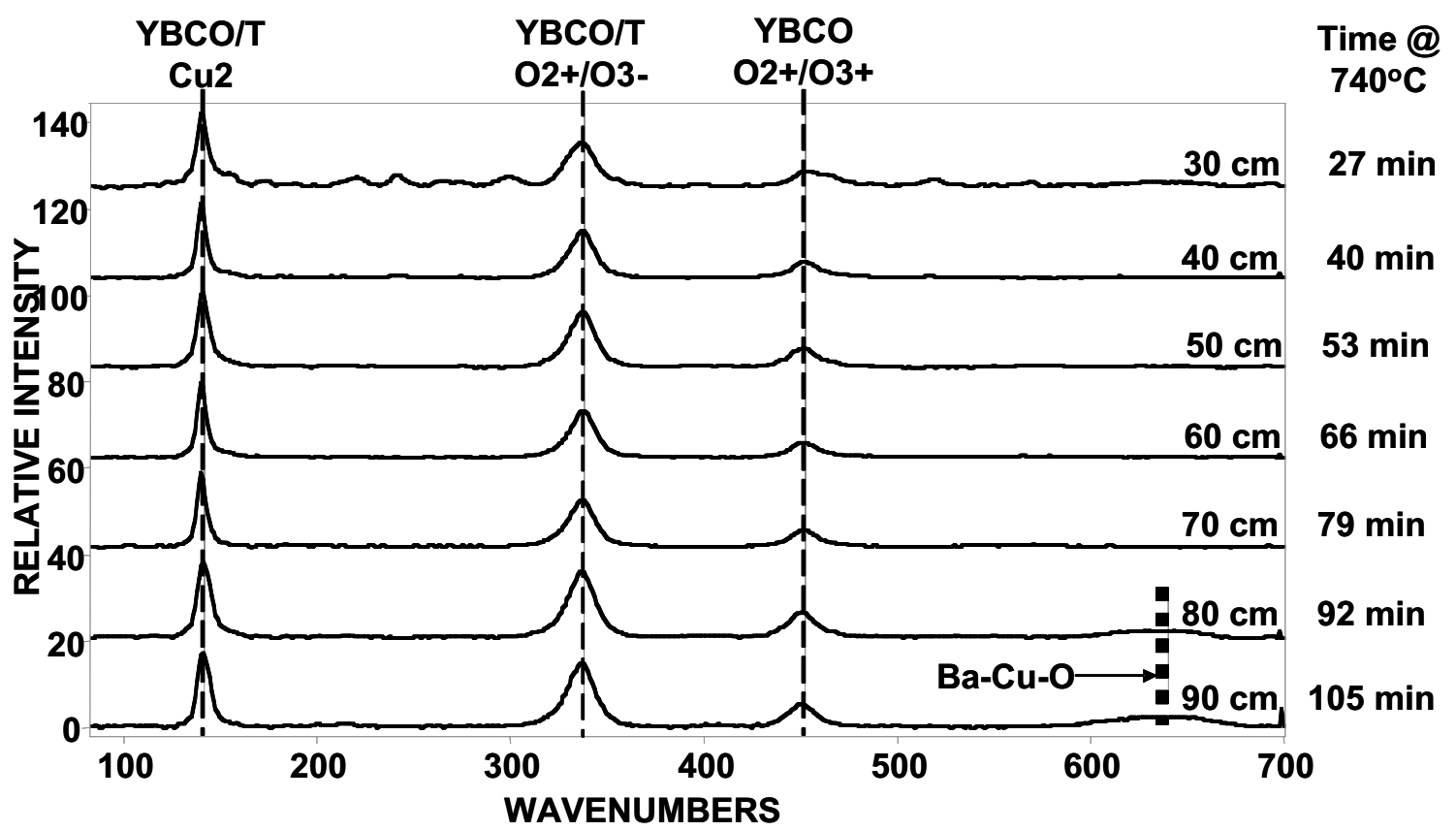

Fig. 52 Raman spectra of increments that range from end of transformation region to beginning of over-processed region along 1.25-m YBCO time-gradient-processed tape. "Sweet spot" appears to occur in range of 50-70 min at $740^{\circ} \mathrm{C}$ for 280-nm-thick YBCO film. 


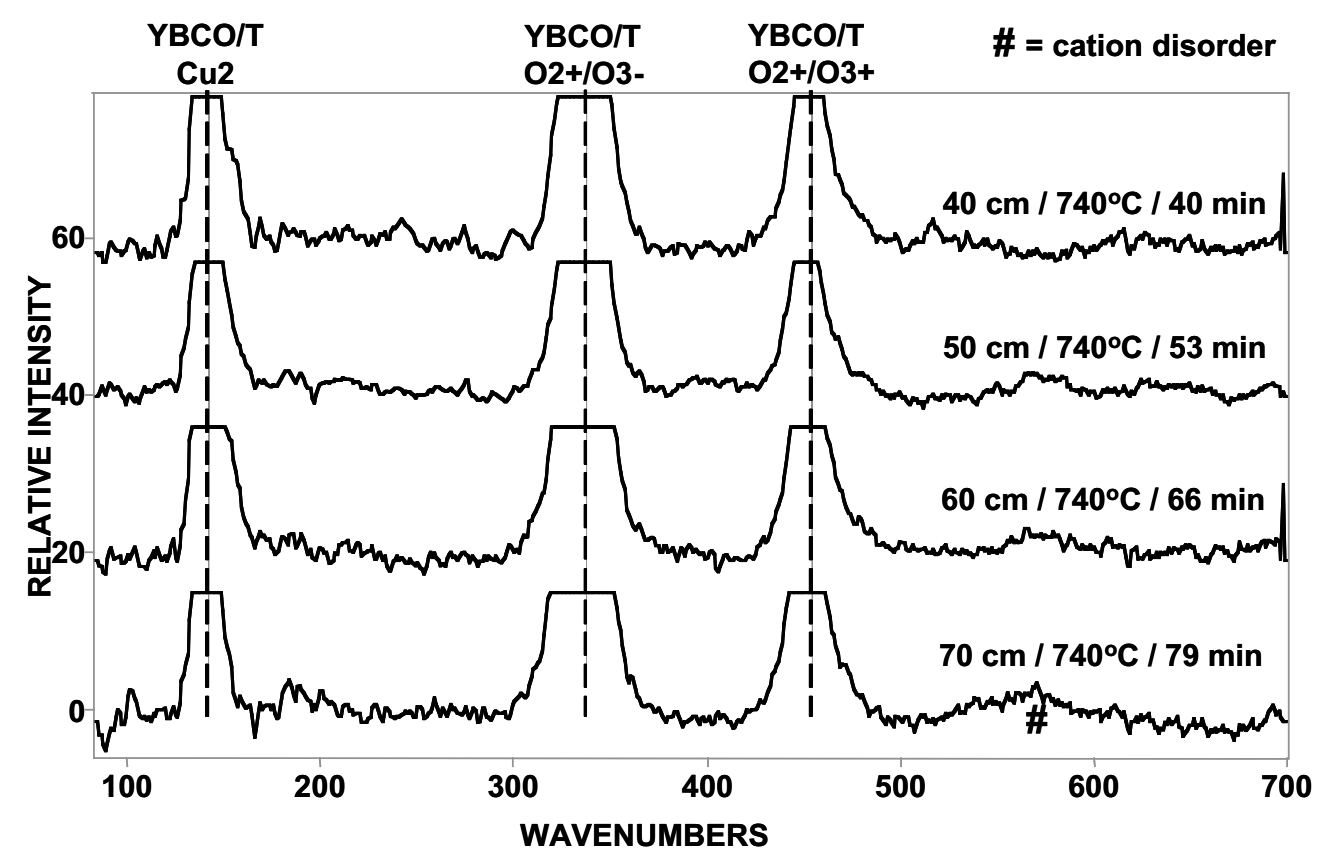

Fig. 53 Intensity-scale-expanded Raman spectra at four increments in region of "sweet spot" along YBCO time-gradient-processed tape. No second phases and only minimal cation disorder can be observed.

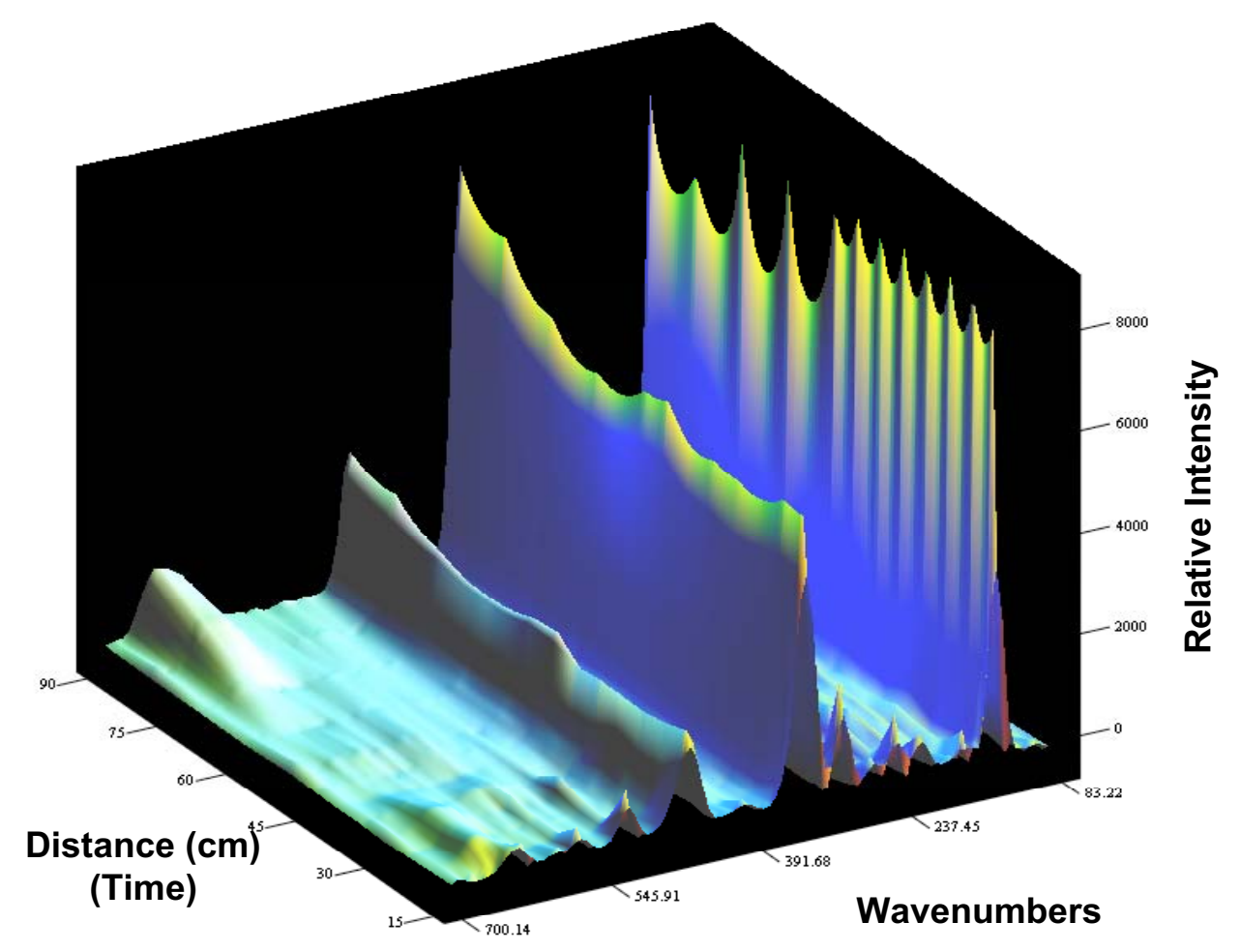

Fig. 54. Raman spectral frequency (wavenumbers) and relative intensity vs. position $(\mathrm{cm})$ along $\approx 300 \mathrm{~nm}$ ORNL TGP tape. Results portrayed here cover most of the tape that experienced the soak temperature $\left(740^{\circ} \mathrm{C}\right)$ for some period of time. 
The histogram in Fig. 54 is dominated by three of the tetragonal YBCO phonons (at $\approx 450 \mathrm{~cm}^{-1}, 330 \mathrm{~cm}^{-1}$, and $145 \mathrm{~cm}^{-1}$ ), but other Raman bands associated with $\mathrm{BaF}_{2}, \mathrm{CuO}, \mathrm{Y}_{2} \mathrm{Cu}_{2} \mathrm{O}_{5}$, and barium cuprates (as identified above) are also clearly present. After forming the families of 3-D histograms for each of the two ORNL TGP tapes, it became clear that the most informative view was the one looking down from above on the distance (time)-wavenumber plane, using gray scale or color to portray intensity. Two such plots for the $\approx 300 \mathrm{~nm}$ YBCO tape specimen are presented in Figs. 55 and 56.

Figure 55 shows the early stages of the reaction process that encompass the ramp-up segment and the first $15 \mathrm{~min}$ at $740^{\circ} \mathrm{C}$. In this plot, all the intensities are normalized to the intensity of the $\mathrm{CuO}$ phonon at $\approx 300 \mathrm{~cm}^{-1}$, because that mode is present at some detectable level across the entire time span of the plot. The two broadband envelopes at the left edge of the plot are due to Raman scattering from the amorphous mush discussed above. This mush dissipates before the soak temperature is reached, leaving only $\mathrm{CuO}$ as a Raman-detectable phase. Quite possibly, this dissipation occurs as a result of the formation of a liquid phase from which the three characteristic tetragonal $\mathrm{YBCO}(\mathrm{YBCO} / \mathrm{T})$ phonons begin to emerge after $\approx 4 \mathrm{~min}$ at $740^{\circ} \mathrm{C}$.

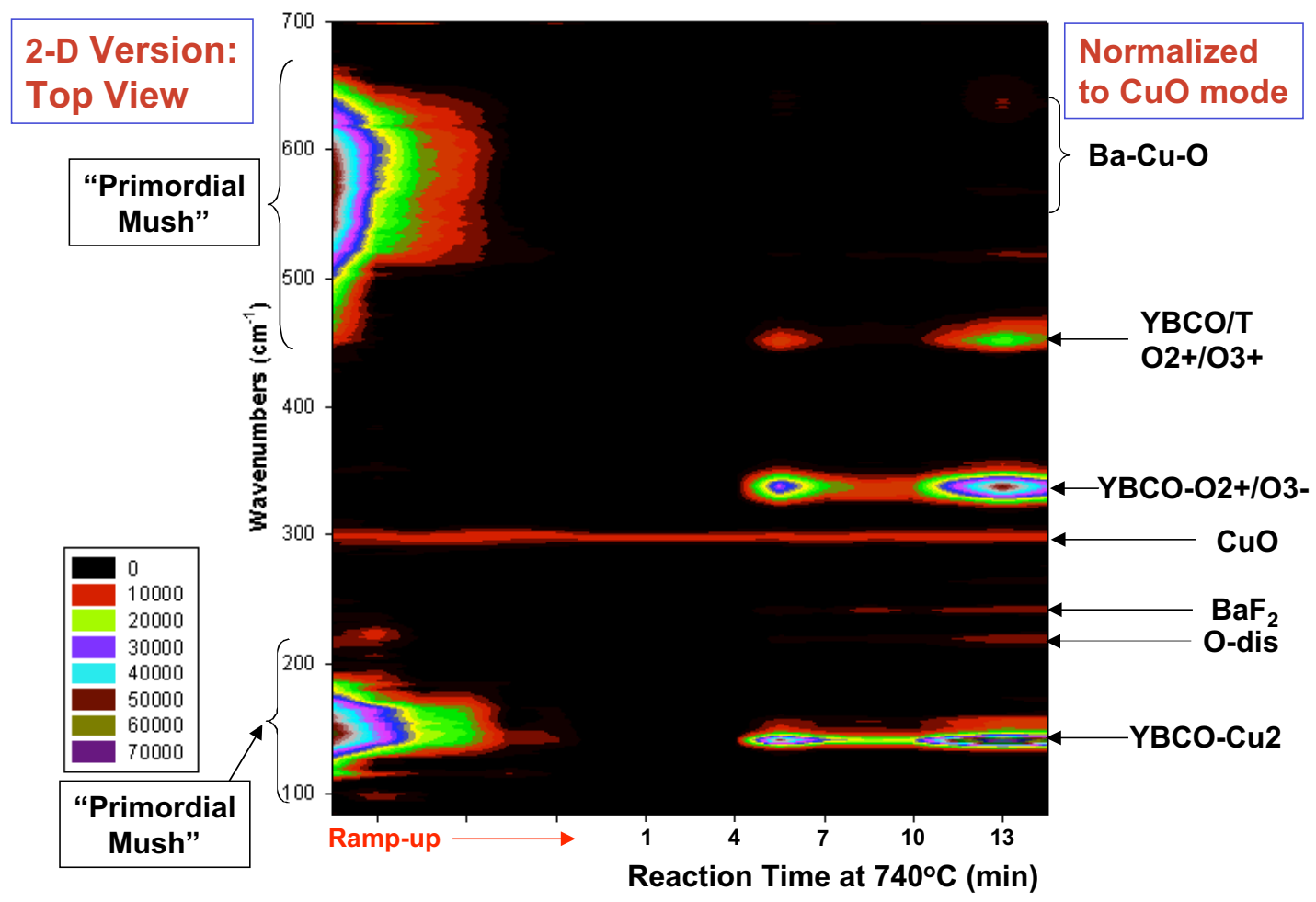

Fig. 55 Top view of Raman frequency-reaction time plane (intensities given by color) for $\approx 300 \mathrm{~nm}$ TGP tape during ramp-up and early soak at $740^{\circ} \mathrm{C}$. Intensities are normalized to that of $\mathrm{CuO}$ mode at $\approx 300 \mathrm{~cm}^{-1}$. 


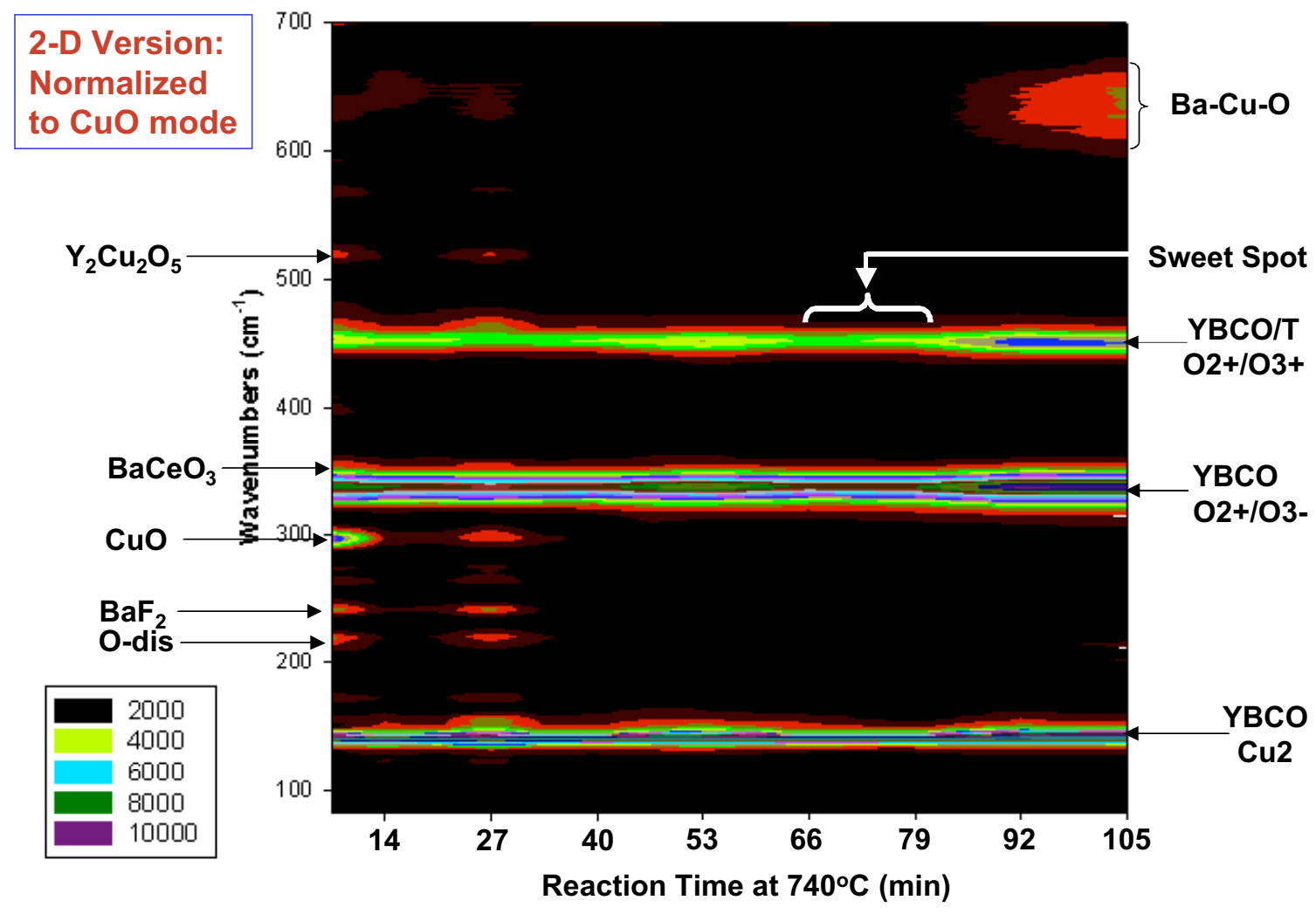

Fig. 56 Top view of Raman frequency-reaction time plane (intensities given by color) for $\approx 300 \mathrm{~nm}$ TGP tape from early to late in soak at $740^{\circ} \mathrm{C}$. Intensities are normalized to that of $\mathrm{Cu} 2$ mode of $Y B C O / T$ at $\approx 145 \mathrm{~cm}^{-1}$.

Figure 56 shows the time-wavenumber plot for the remainder of the $\approx 300$ $\mathrm{nm}$ precursor tape (from $10 \mathrm{~min}$ at $740^{\circ} \mathrm{C}$ to the over-processed domains at the end of the precursor coated segment). In this plot, all the Raman band intensities are normalized to the $\mathrm{Cu} 2\left(145 \mathrm{~cm}^{-1}\right)$ mode of $\mathrm{YBCO} / \mathrm{T}$ because the $\mathrm{Cu} 2$ mode is persistently present over this entire segment of the tape. Moving from left to right in this plot, it is noteworthy that (1) Raman bands associated with $\mathrm{BaCeO}_{3}$, $\mathrm{BaF}_{2}, \mathrm{CuO}, \mathrm{Y}_{2} \mathrm{Cu}_{2} \mathrm{O}_{5}$, and barium cuprates vanish after approximately $30 \mathrm{~min}$ at $740^{\circ} \mathrm{C}$, (2) there is an intermediate time domain from 30 to $90 \mathrm{~min}$ at $740^{\circ} \mathrm{C}$ wherein the three $\mathrm{YBCO} / \mathrm{T}$ phonons sharpen up to form the indicated "sweet spot" associated with the optimum performance point for this particular wire as demonstrated by Lee et al. at ORNL, and (3) beyond the $90 \mathrm{~min}$ at $740^{\circ} \mathrm{C}$, the three $\mathrm{YBCO} / \mathrm{T}$ phonons experience broadening along with the reappearance of barium cuprate (Ba-Cu-O) phonons, indicating the onset of $\mathrm{YBCO}$ decomposition presumably due to over-processing. Two other weak Raman bands that appear during the early stages of YBCO formation, indicated as C-dis and O-dis in Figs. 55 and 56, are ascribed to cation disorder and oxygen atom disorder, respectively, in the YBCO lattice. Their presence in the early stages of YBCO formation is both understandable and expected. 
Plots comparable to Figs. 55 and 56 were also constructed from R2R Raman data for the $\approx 1000 \mathrm{~nm}$ precursor tape specimen. The results and corresponding conclusions are tacitly the same as for the $\approx 300 \mathrm{~nm}$ precursor, with the expected exception that the thrice-thicker precursor took approximately three times longer at $740^{\circ} \mathrm{C}$ to reach the "sweet spot" condition. Figure 57 shows the plot for the $\approx 1000 \mathrm{~nm}$ precursor specimen that corresponds to the conditions/ results in Fig. 56. The non-YBCO spectral features seen in Fig. 56 are present in Fig. 57 as well, and actually appear with greater vividness in most cases.

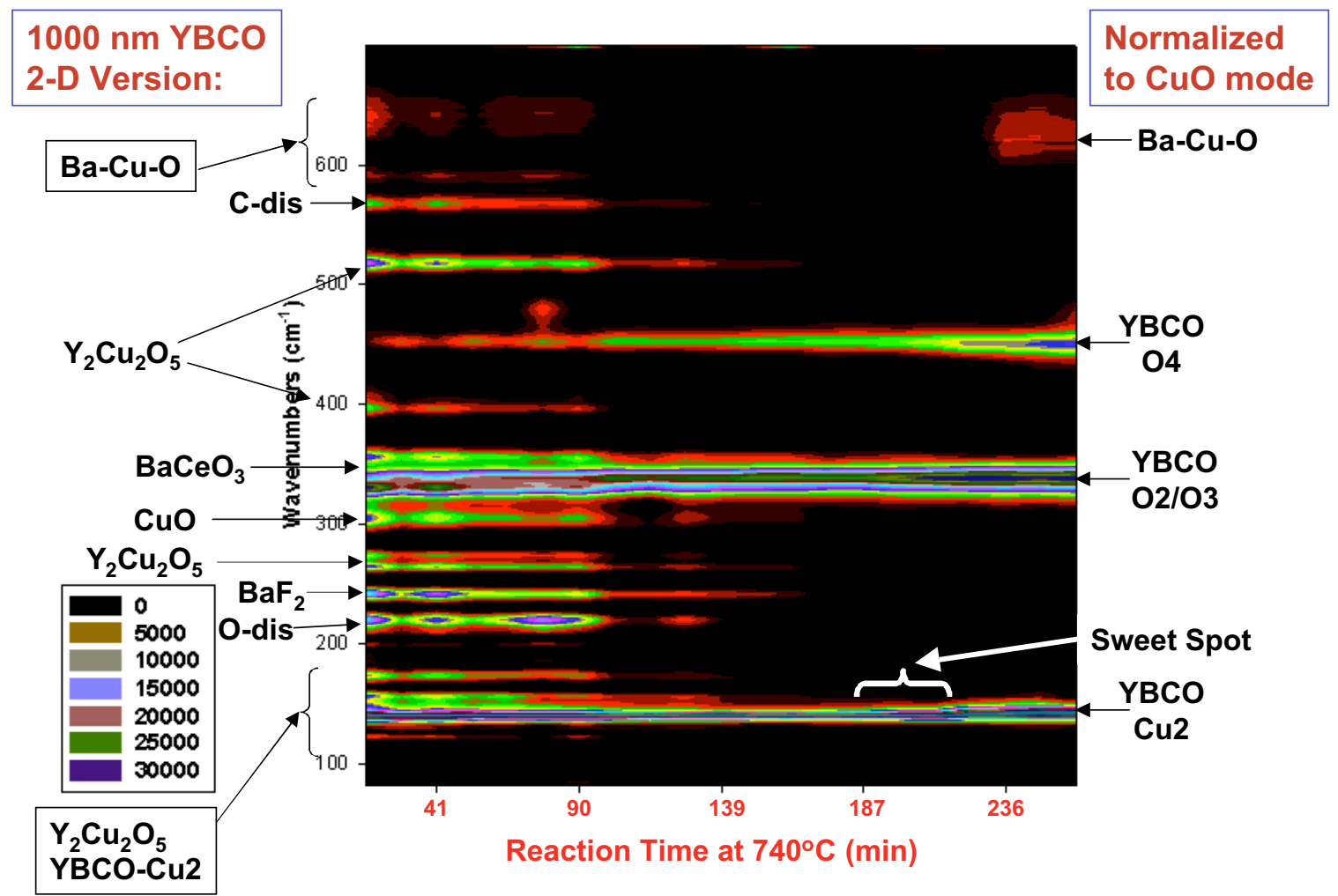

Fig. 57 Top view of Raman frequency-reaction time plane (intensities given by color) for $\approx 1000 \mathrm{~nm}$ TGP tape from early to late in soak at $740^{\circ} \mathrm{C}$. Intensities are normalized to that of Cu2 mode of $Y B C O / T$ at $\approx 145 \mathrm{~cm}^{-1}$.

\subsubsection{Oxygen Doping of YBCO Grain Boundaries}

Grain boundaries with misorientation angles larger than $\approx 5^{\circ}$ can severely limit the critical current of coated conductors. There are, in principle, two ways to address this problem. One is to avoid producing such grain boundaries during deposition of the superconductor, but this becomes more difficult as the length and thickness of conductors increase; the other is to enhance the properties of the grain boundaries by doping, e. g., by secondary oxygenation treatments. In our study we observed that in the over-doped regime, where the superconducting transition temperature $\left(T_{c}\right)$ of the films decreases with 
increasing oxygen doping, the critical current density $\left(\mathrm{J}_{\mathrm{c}}\right)$ of the grain boundaries continues to increase. Thus films with the best $\mathrm{J}_{\mathrm{c}}$ values do not necessarily exhibit the highest $\mathrm{T}_{\mathrm{c}}$. If oxygen doping proves successful in enhancing the properties of low angle grain boundaries, it could greatly simplify the production of high-quality coated conductors.

In an effort to improve critical currents of coated conductors by oxygen doping of grain boundaries, we are developing oxygenation techniques for artificial grain boundaries in $\mathrm{YBCO}$ films on $\mathrm{SrTiO}_{3}$ bicrystals and will then apply these techniques to coated conductors. In this study, we used YBCO films deposited on $\mathrm{SrTiO}_{3}$ bicrystals with [001] tilt grain boundaries (GBs) between $0^{\circ}$ and $24^{\circ}$. By varying the oxygenation temperature between 500 and $300^{\circ} \mathrm{C}$, we changed the oxygen concentration of a given film from the under-doped to the over-doped regime. The $\mathrm{J}_{\mathrm{c}}$ was determined by a contact-free method from the maximum persistent current that can be induced in a thin-film ring containing two segments of the grain boundary.

Rings without secondary oxygenation treatments We investigated several rings on single crystal substrates and on bicrystal substrates with GB angles of $5^{\circ}$, $10^{\circ}, 15^{\circ}$, and $24^{\circ}$. Our contact-free method for measuring the $\mathrm{J}_{\mathrm{c}}$ is especially advantageous for very high critical currents like those in rings with small angle GBs. In a single-crystal thin-film ring of thickness $200 \mathrm{~nm}$ and width $0.5 \mathrm{~mm}$, the critical current at low temperature can be as high as $50 \mathrm{~A}$, corresponding to a $\mathrm{J}_{\mathrm{C}}$ of $5 \times 10^{7} \mathrm{~A} / \mathrm{cm}^{2}$. Such a large current can be induced in the thin-film ring with a magnetic field of only $\approx 30 \mathrm{mT}$. Typically, $\mathrm{J}_{\mathrm{c}}$ for single crystal films varied from 3 $\times 10^{6} \mathrm{~A} / \mathrm{cm}^{2}$ at $77 \mathrm{~K}$ to $4 \times 10^{7} \mathrm{~A} / \mathrm{cm}^{2}$ at $4.2 \mathrm{~K}$. Rings containing a $5^{\circ} \mathrm{GB}$ exhibited $\mathrm{J}_{\mathrm{C}}$ values that were very similar to those of the single crystal films.

Figure 58 shows the critical current at $77 \mathrm{~K}$ as function of GB angle for rings in the as-made state, i.e. without a secondary oxygenation treatment. These results are in good agreement with literature values published previously. There are two distinct regions in this plot: $0-5^{\circ}$ where $\mathrm{J}_{\mathrm{c}}$ is independent of the angle, being limited by the film material, not the $G B$, and $>5^{\circ}$ where $\mathrm{J}_{\mathrm{c}}$ drops exponentially with GB angle, now being limited by the rapidly deteriorating GBs. In the crossover between these two regions, at $\approx 5-10^{\circ}$, secondary oxygen treatments are expected to be the most beneficial for coated conductors.

The crossover from film-limited $\mathrm{J}_{\mathrm{C}}$ to GB-limited $\mathrm{J}_{\mathrm{C}}$ shown in Fig. 58 as function of GB angle, can also be seen as function of temperature. This is demonstrated in Fig. 59, which shows the current density of a film with a strong $10^{\circ} \mathrm{GB}$. The ring was cooled in zero field to $<50 \mathrm{~K}$. A magnetic field of $1 \mathrm{mT}$ was then applied to induce a current of $1.55 \mathrm{~A}$, corresponding to a current density, J, of $1.55 \times 10^{6} \mathrm{~A} / \mathrm{cm}^{2}$. 


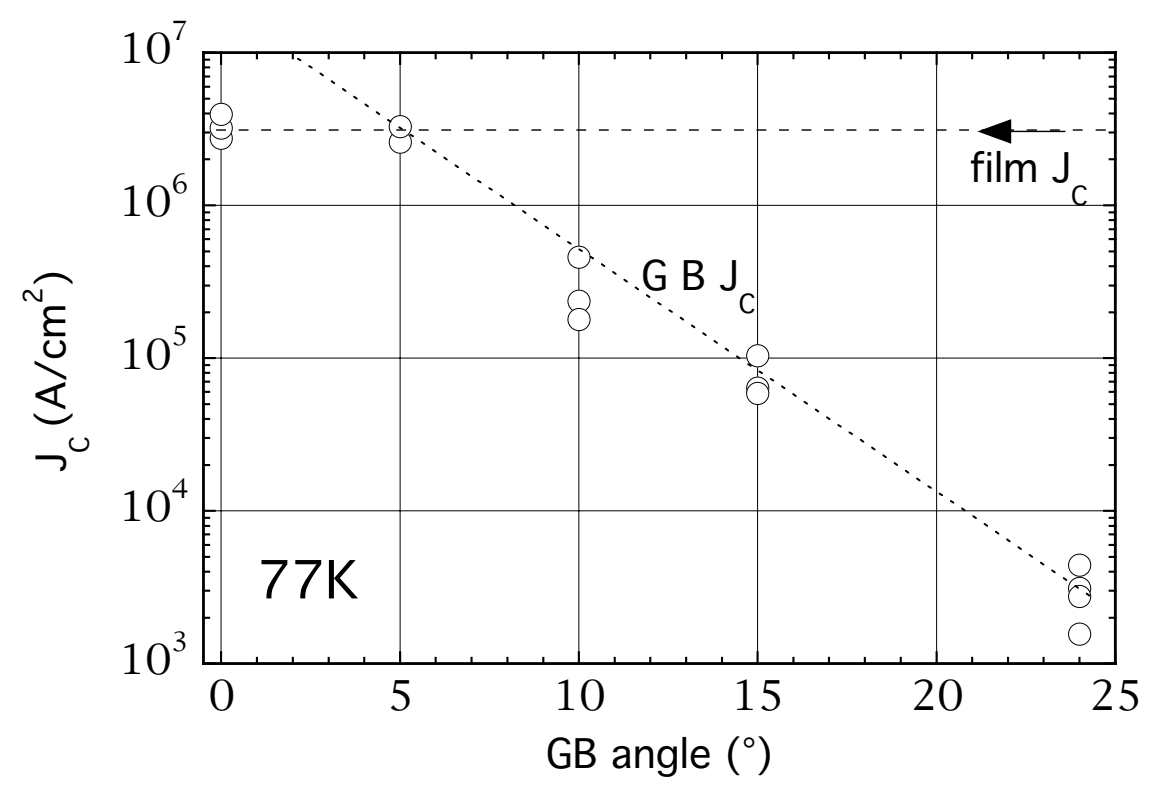

Fig. 58 Critical current densities of all GBs investigated vs. GB angle. Horizontal dashed line represents $J_{c}$ of the film, dotted line that of the GBs. With increasing $G B$ angle, a crossover from film-limited $J_{c}$ to GB-limited $J_{C}$ occurs near $5^{\circ}$.

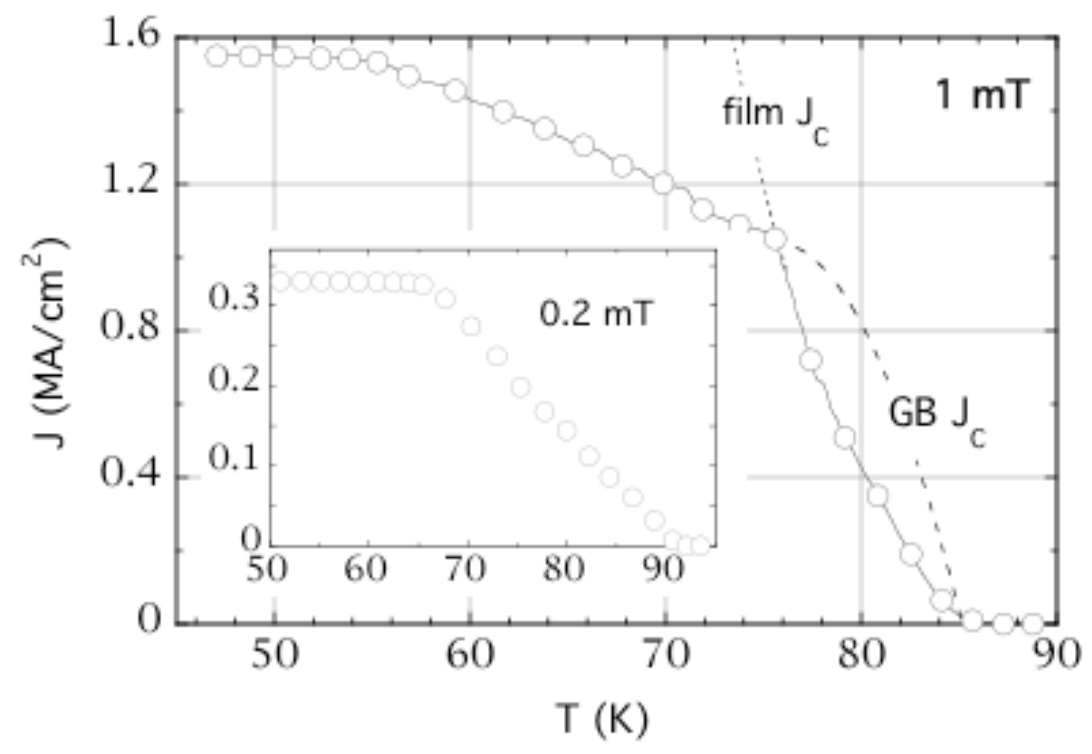

Fig. 59 Persistent current in ring containing $10^{\circ} \mathrm{GB}$. Applying magnetic field of 1 $m T$ at low temperature induces current density of $1.55 \mathrm{MA} / \mathrm{cm}^{2}$ in the ring. Kink at $56 \mathrm{~K}$ signals that temperature-dependent $G B$ critical current limits persistent current above this temperature. Second kink at $\approx 75 \mathrm{~K}$ signals crossover to film-limited persistent current. Dotted and dashed lines estimate temperature dependence of film and GB critical currents, respectively. Inset displays persistent current of ring with "normal" $10^{\circ} \mathrm{GB}$. Units of the inset are identical to those of main figure. 
As the temperature is raised (Fig. 59), J initially remains constant until $\mathrm{J}_{\mathrm{C}, \mathrm{BB}}(\mathrm{T})$, the critical current density of the $\mathrm{GB}$, falls below this value (at $\approx 55 \mathrm{~K}$ ). As the temperature is further increased, $\mathrm{J}_{\mathrm{C}, \mathrm{CB}}$ limits the persistent current flowing in the ring. For most GBs, $\mathrm{J}_{\mathrm{C}, \mathrm{CB}}$ decreases almost linearly with temperature and drops to zero near the $\mathrm{T}_{\mathrm{c}}$ of the film. However, Fig. 59 shows a second kink for this particular ring at $\approx 75 \mathrm{~K}$. Above this temperature, $\mathrm{J}_{\mathrm{C}}$ of the film, $\mathrm{J}_{\mathrm{c}_{\text {, film }}}$ becomes smaller than $\mathrm{J}_{\mathrm{C}, \mathrm{BB}}$. The dashed and dotted lines represent extrapolations that indicate how $\mathrm{J}_{\mathrm{C}, \mathrm{BB}}$ and $\mathrm{J}_{\mathrm{C} \text {, film }}$ might vary with temperature $\left(\mathrm{J}_{\mathrm{C}, \mathrm{CB}}\right.$ must reach zero near $\mathrm{T}_{\mathrm{c}}$ for the film, $88.1 \mathrm{~K}$ ). The inset in Fig. 59 shows how $\mathrm{J}$ varies with temperature for another, weaker $10^{\circ} \mathrm{GB}$. In this ring, a J of $0.33 \mathrm{MA} / \mathrm{cm}^{2}$ was originally induced below $50 \mathrm{~K}$ by an applied field of $0.2 \mathrm{mT}$. Here $\mathrm{J}_{\mathrm{C}, \mathrm{GB}}$ varies nearly linearly with $\mathrm{T}$ all the way up to the $\mathrm{T}_{\mathrm{C}}$ of the film $(91.7 \mathrm{~K})$, which was typical of most GBs that were investigated.

Figure 60 shows $\mathrm{J}_{\mathrm{C}, \mathrm{GB}}$ for four rings containing $10^{\circ} \mathrm{GBs}$. All data are for rings in the as-made state, i.e., without any secondary oxygenation treatments. As can be seen, there is considerable variation in $\mathrm{J}_{\mathrm{C}, \mathrm{GB}}$ of the rings, with a clear correlation between $J_{c}$ and $T_{c}$ of the films $\left(T_{c}\right.$ values are listed in Fig. 60 next to the ring number). The ring with the highest $\mathrm{T}_{\mathrm{c}}$ has the lowest $\mathrm{J}_{\mathrm{c}}$ and vice versa. Figure 61 shows $J_{C}$ at $77 \mathrm{~K}$ as function of $T_{C}$ of the four rings. There is nearly a factor of three difference between the lowest and highest $\mathrm{J}_{\mathrm{c}}$. The origin of this difference is not known. All films are nominally made in the same way. The relatively large variation in $T_{c}$ could be due to a variation in the oxygen concentration that results, for example, from seemingly minor differences in the cool-down procedures after film growth. It is therefore of interest to systematically investigate how changing the oxygen concentration affects $\mathrm{J}_{\mathrm{C}}$ of the GBs.

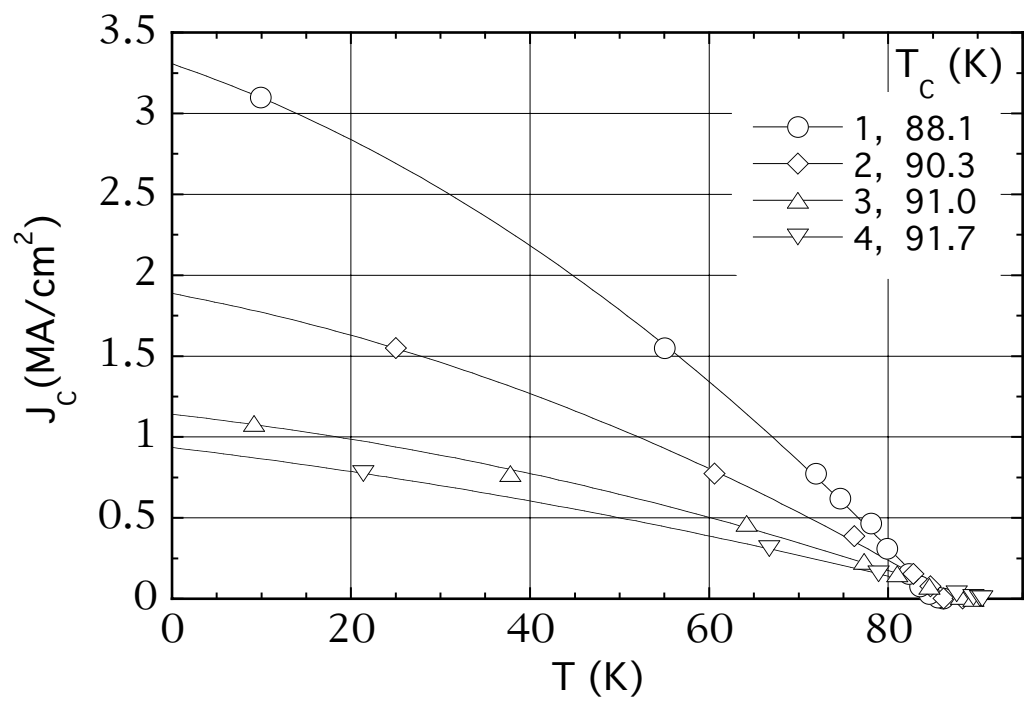

Fig. $60 J_{c}(T)$ for four rings each containing a $10^{\circ} \mathrm{GB}$. Listed next to the ring number are the $T_{c}$ values of the film material for that ring. 


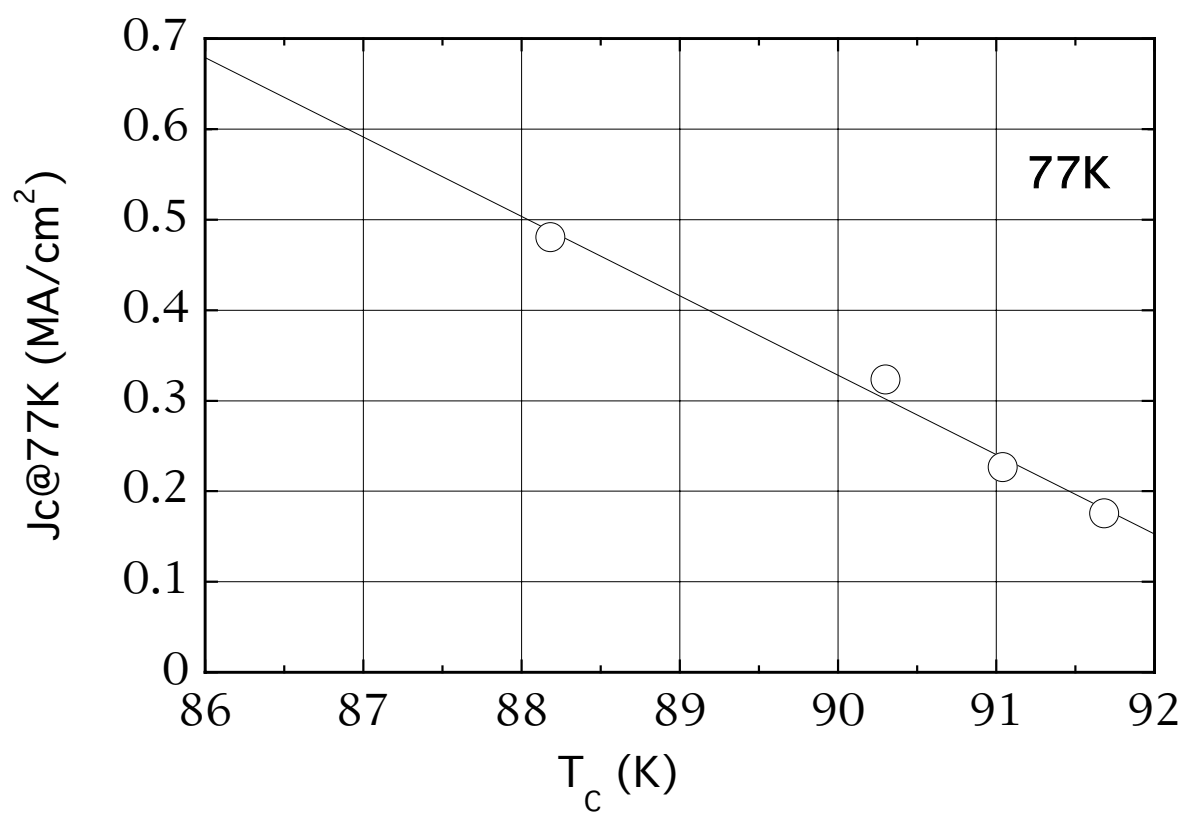

Fig. 61. $J_{c}$ at $77 \mathrm{~K}$ vs. $T_{c}$ for the four rings with $10^{\circ} \mathrm{GBs}$ shown in Fig. 60.

Effect of oxygen doping To change the oxygen concentration of the thinfilm rings, they are placed inside a container made of melt-textured $\mathrm{YBCO}$ and annealed in flowing oxygen at temperatures between 500 and $350^{\circ} \mathrm{C}$. The annealing times range from one hour to several days. From the variation in the superconducting transition temperature of the films, we can estimate the oxygen concentration through comparison with YBCO single crystal work. We do not know, however, the oxygen concentration inside the GBs. We assume it varies along with the oxygen concentration of the film.

Most previous doping studies on GBs were made on $24^{\circ}$ GBs. To compare the results of oxygen doping with previous work, we systematically investigated how the critical current of $24^{\circ}$ GBs varies with oxygen doping. Figure 62 displays $\mathrm{J}_{\mathrm{C}}$ vs. $\mathrm{T}$ for a $24^{\circ} \mathrm{GB}$ ring with rather low $\mathrm{J}_{\mathrm{c}}$ in the as-made state. After secondary oxygenation treatments at $450^{\circ} \mathrm{C}$ and $400^{\circ} \mathrm{C}, \mathrm{T}_{\mathrm{C}}$ systematically decreases from $91.7 \mathrm{~K}$ in the as-made state to $90.8 \mathrm{~K}$ after oxygenation at $400^{\circ} \mathrm{C}$ (the $\mathrm{T}_{\mathrm{c}}$ values are listed in Fig. 62, next to the oxygenation temperature). This trend clearly indicates that the film is in the over-doped state, because $T_{C}$ decreases with increasing oxygenation level. Over-doping the film is accompanied by a dramatic increase in $\mathrm{J}_{\mathrm{C}}$ as seen in Fig. 62. For example, $\mathrm{J}_{\mathrm{C}}$ at 77 $\mathrm{K}$ increases from $1.5 \mathrm{kA} / \mathrm{cm}^{2}$ in the as-made state to $17.4 \mathrm{kA} / \mathrm{cm}^{2}$ after annealing at $400^{\circ} \mathrm{C}$, more than a factor of ten. Oxygenation increased $\mathrm{J}_{\mathrm{C}}$ at $77 \mathrm{~K}$ by smaller factors $(\approx 5)$ for other $24^{\circ} \mathrm{GBs}$. We expect that annealing below $400^{\circ} \mathrm{C}$ should 
further increase the oxygen concentration, resulting in lower $\mathrm{T}_{\mathrm{c}}$ and even higher $\mathrm{J}_{\mathrm{c}}$ values. However, in the present investigation, we observed a degradation of the GBs after repeated oxygenation treatments resulting in irreversible decrease in $\mathrm{J}_{\mathrm{c}}$. We believe that residues on the film left over from the photolithographic process cause this degradation. In the future we will produce the film rings by a masking technique, thus avoiding this contamination. From single crystal work, it is known that $\mathrm{T}_{\mathrm{c}}$ can be reduced to $\approx 87 \mathrm{~K}$ by over-doping. We thus can expect an even larger increase in $\mathrm{J}_{\mathrm{c}}$ if we can avoid degradation of the GBS.

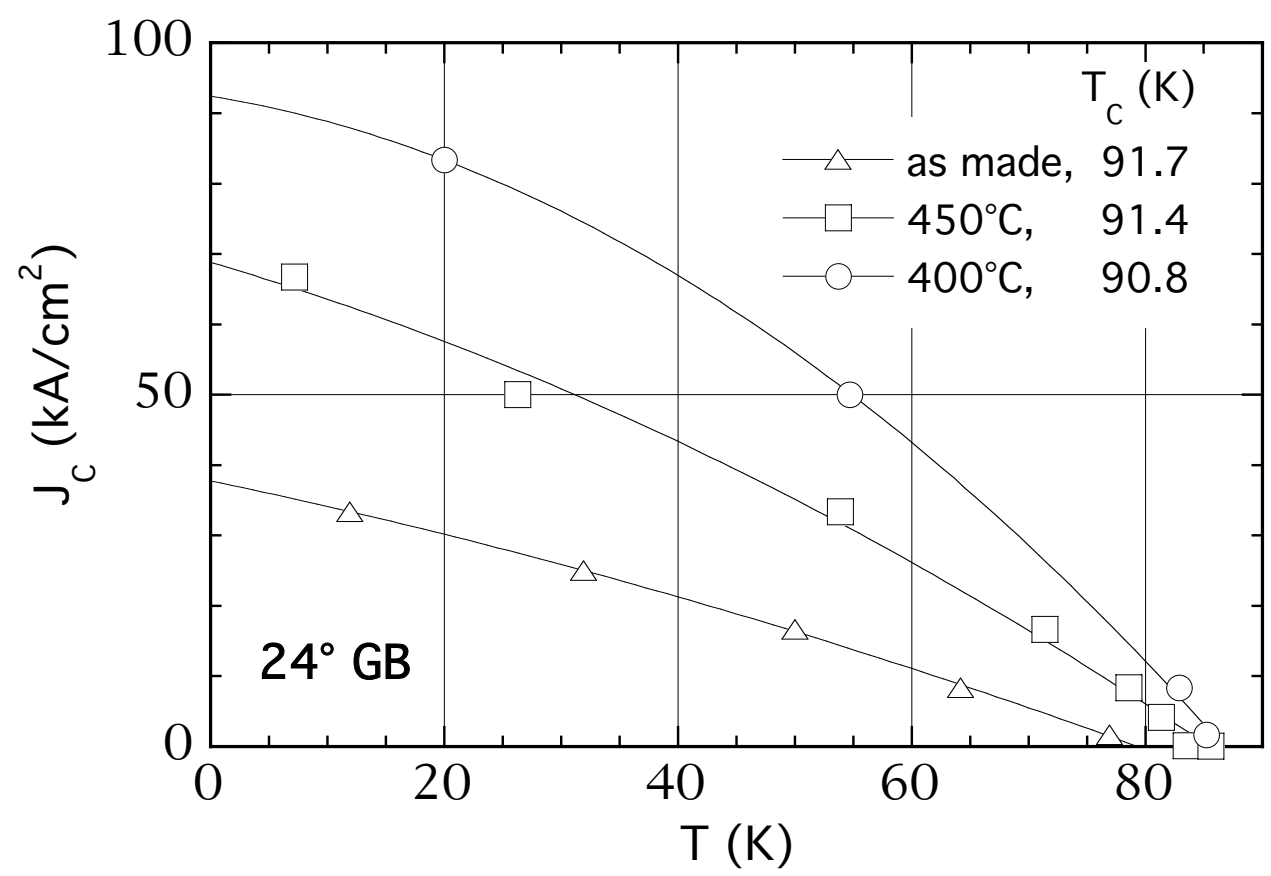

Fig. $62 J_{c}(T)$ of $24^{\circ} \mathrm{GB}$ in as-made state and after secondary oxygenation at $450^{\circ} \mathrm{C}$ and $400^{\circ} \mathrm{C}$. Film $T_{c}$ values in the various states are also listed.

Secondary oxygenation treatments also improved the $\mathrm{J}_{\mathrm{C}}$ of rings with 10 and $15^{\circ}$ GBs but not as dramatically as for the $24^{\circ}$ GB. Figs. 63 and 64 show how $\mathrm{J}_{\mathrm{C}}$ increases with increasing oxygen doping. The oxygenation temperatures are listed in the figures together with the $T_{c}$ values. In both cases, $T_{c}$ decreases by more than $2 \mathrm{~K}$, indicating that the films are strongly overdoped, but $\mathrm{J}_{\mathrm{c}}$ increases only marginally. As with films with $24^{\circ} \mathrm{GBs}$, we observed irreversible degradation of the GBs after repeated oxygenation treatments. Oxygenation is expected to produce much more significant increases in $\mathrm{J}_{\mathrm{C}}$ if the degradation can be avoided. 


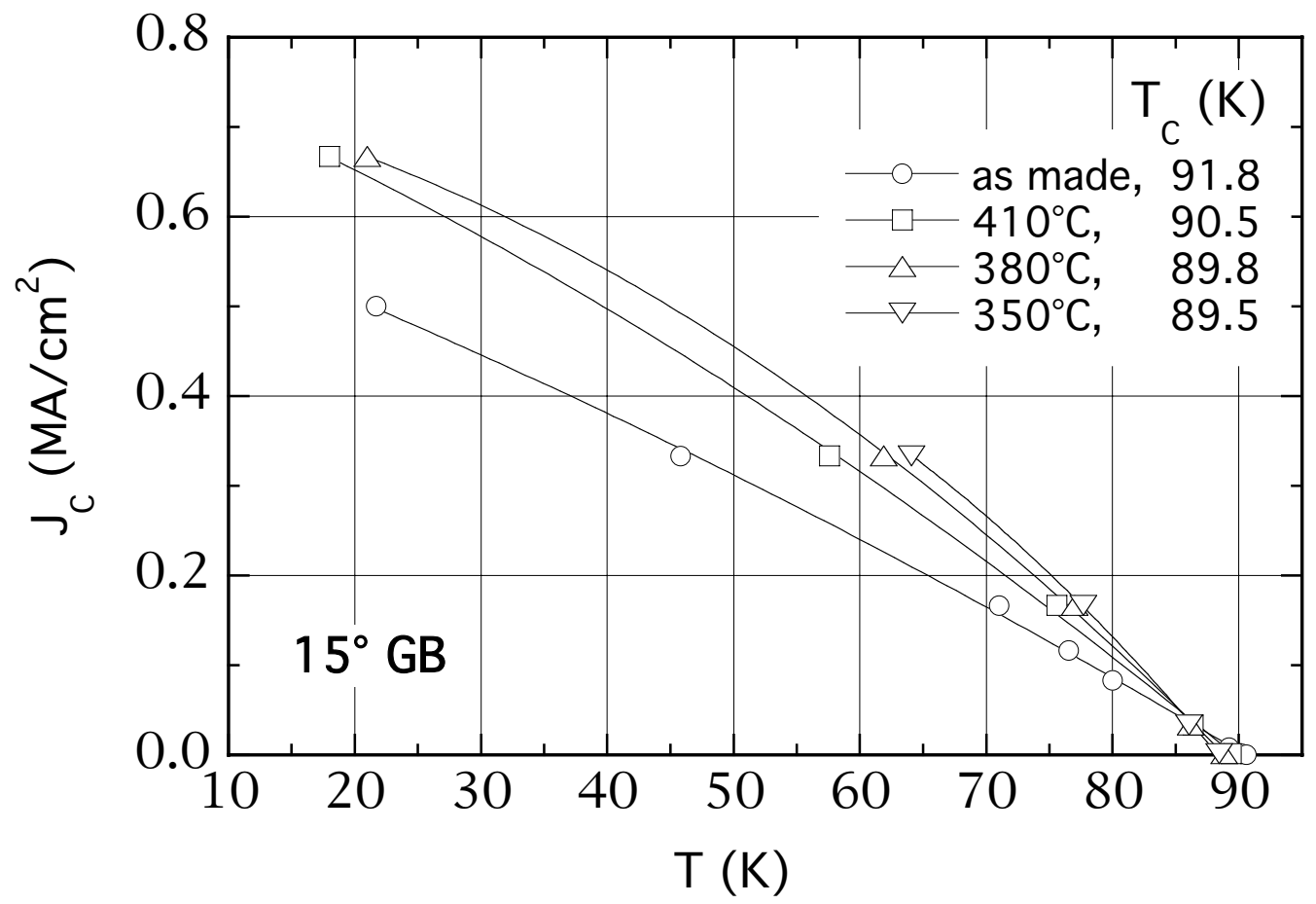

Fig. $63 J_{c}(T)$ of a $15^{\circ} \mathrm{GB}$ in the as-made state and after secondary oxygenation at $410^{\circ} \mathrm{C}, 380^{\circ} \mathrm{C}$ and $350^{\circ} \mathrm{C}$. Film $T_{c}$ values in the various states are also listed.

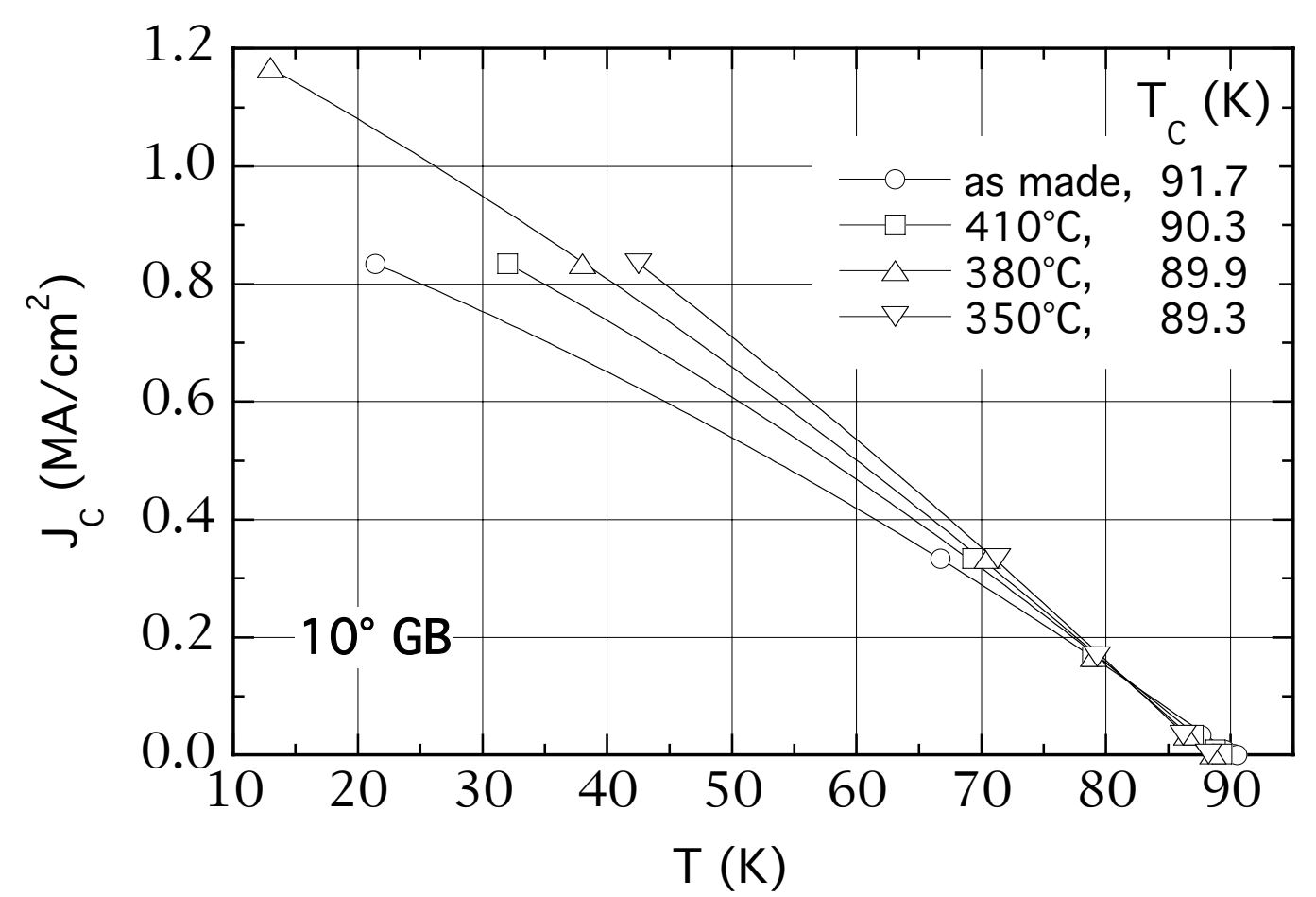

Fig. 64. $J_{c}(\mathrm{~T})$ of a $10^{\circ} \mathrm{GB}$ in the as-made state and after secondary oxygenation at $410^{\circ} \mathrm{C}, 380^{\circ} \mathrm{C}$ and $350^{\circ} \mathrm{C}$. Film $T_{c}$ values in the various states are also listed. 


\subsubsection{Mechanical Properties of Coated Conductors}

Coated conductors will be subjected to various stresses during their application, e.g., bending stresses that result from the winding of coils or stresses arising from Lorentz forces during the passage of currents. Such stresses may cause microcracking in the superconductor layer, possibly degrading its superconducting properties and reducing its service life. It is, therefore, important to assess the ability of coated conductors to withstand expected operational stresses. In addition, processing of the multi-layer coated conductor introduces residual stresses in the superconductor and other layers, which affect the service life and performance of the conductor. In FY 2003, we began to address these issues by measuring the strain tolerance of YBCO in ISD-based coated conductors, and we initiated an effort to evaluate the residual stresses in their individual layers.

\subsection{6.a Strain Tolerance of YBCO in ISD-Based Conductors}

Coated conductors will encounter mechanical strains/stresses during both processing and service. Because these strains/stresses may damage the superconductor and possibly degrade or destroy its superconducting properties, it is important to evaluate the critical value of strain (strain tolerance) at which conductors exhibit degradation in superconductivity, and subsequently, develop an understanding of the relationship between strain tolerance and various processing parameters.

As our initial focus in this area, we evaluated the effect of the YBCO thickness on the tensile bend-strain tolerance of multilayer coated conductors. Specifically, coated conductors were fabricated with the standard ISD architecture: $\mathrm{HC} / \mathrm{ISD} \mathrm{MgO} / \mathrm{HE} \mathrm{MgO} / \mathrm{YSZ} / \mathrm{CeO}_{2} / \mathrm{YBCO}$. For this study, the thickness of the $\mathrm{HC}$ substrate, ISD and $\mathrm{HE} \mathrm{MgO}$ layers, and the $\mathrm{YSZ}$ and $\mathrm{CeO}_{2}$ buffer layers were kept constant, and the thickness of the YBCO layer was varied from 0.1 to $0.5 \mu \mathrm{m}$. The ISD-MgO layer was deposited at room temperature by the electron beam evaporation technique at a deposition angle of $55^{\circ}$ and had a thickness of $1 \mu \mathrm{m}$. The HE-MgO layer $\left(0.5 \mu \mathrm{m}\right.$ thick) was deposited at $700^{\circ} \mathrm{C}$ at a deposition angle of $0^{\circ}$ using the same ISD system. The $\approx 0.1-0.2-\mu$ m-thick YSZ and $\approx 10$-nm-thick $\mathrm{CeO}_{2}$ layers were deposited at $800^{\circ} \mathrm{C}$ by PLD. The YBCO layer was deposited at $760^{\circ} \mathrm{C}$ by the PLD method.

Strain tolerance of the ISD coated conductors was measured by first subjecting the conductor to increasing levels of tensile bend strain $(\varepsilon)$, followed by measuring critical current $\left(\mathrm{I}_{\mathrm{C}}\right)$ by the inductive method. The critical current $\left(\mathrm{I}_{\mathrm{C}}\right)$ of the strained conductors, normalized with respect to the critical current $\left(\mathrm{I}_{\mathrm{c} 0}\right)$ in the unstrained condition, is plotted as a function of applied bend strain $(\varepsilon)$ in Fig. 65. For a given YBCO layer thickness, the critical strain $\left(\varepsilon_{\mathrm{cr}}\right)$ was taken as the strain at which the conductor exhibited the onset of degradation in $\mathrm{I}_{\mathrm{C}}$. The variation of $\varepsilon_{\mathrm{cr}}$ with YBCO layer thickness is shown in Fig. 66. Also, plotted in 
the figure are critical strain values that were calculated analytically by using an assumed toughness of $0.4 \mathrm{MPa} \sqrt{\mathrm{m}}$ for the $\mathrm{YBCO}$. As seen in the figure, the measured $\varepsilon_{\mathrm{cr}}$ decreased from $0.39 \%$ for YBCO with a thickness of $0.2 \mu \mathrm{m}$ to $0.17 \%$ for YBCO with a thickness of $0.5 \mu \mathrm{m}$. This inverse relationship between $\varepsilon_{\mathrm{cr}}$ and YBCO layer thickness is consistent with the analytical prediction.

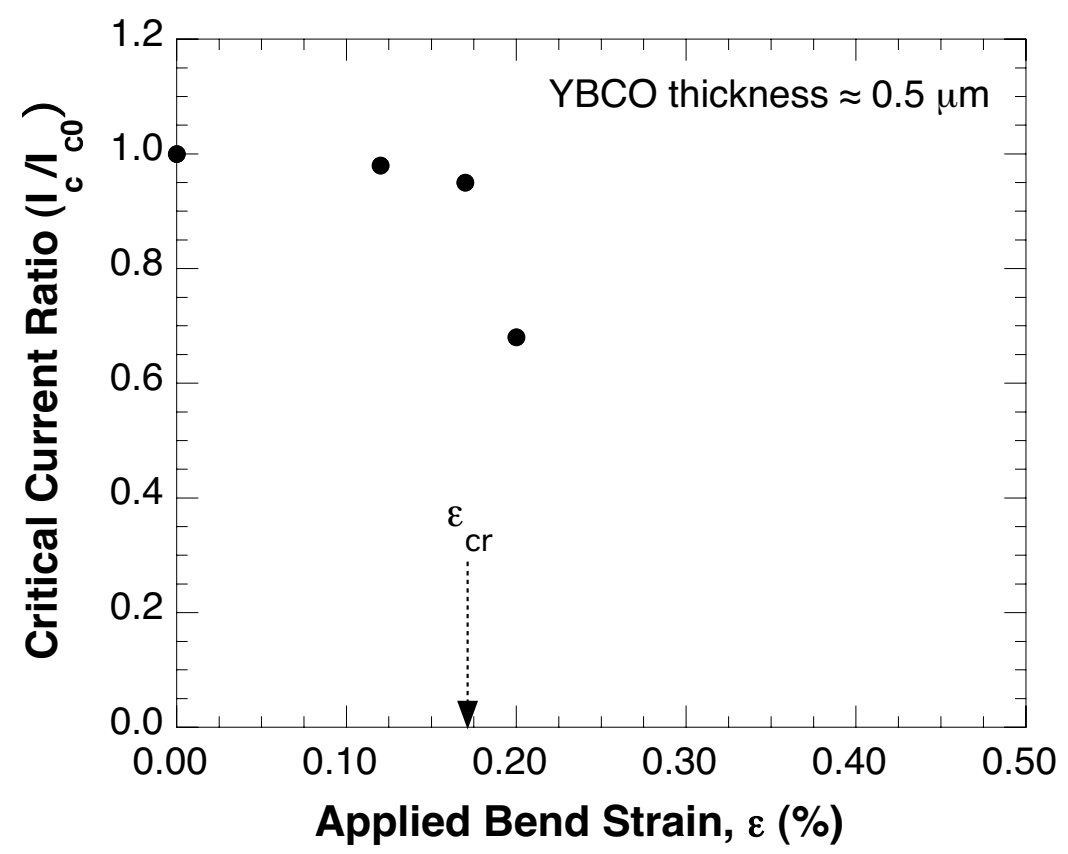

Fig. 65 Variation of critical current ratio $\left(I_{c} / I_{c 0}\right)$ as a function of applied bend strain $\varepsilon$. The critical strain $\varepsilon_{c r}=0.17 \%$.

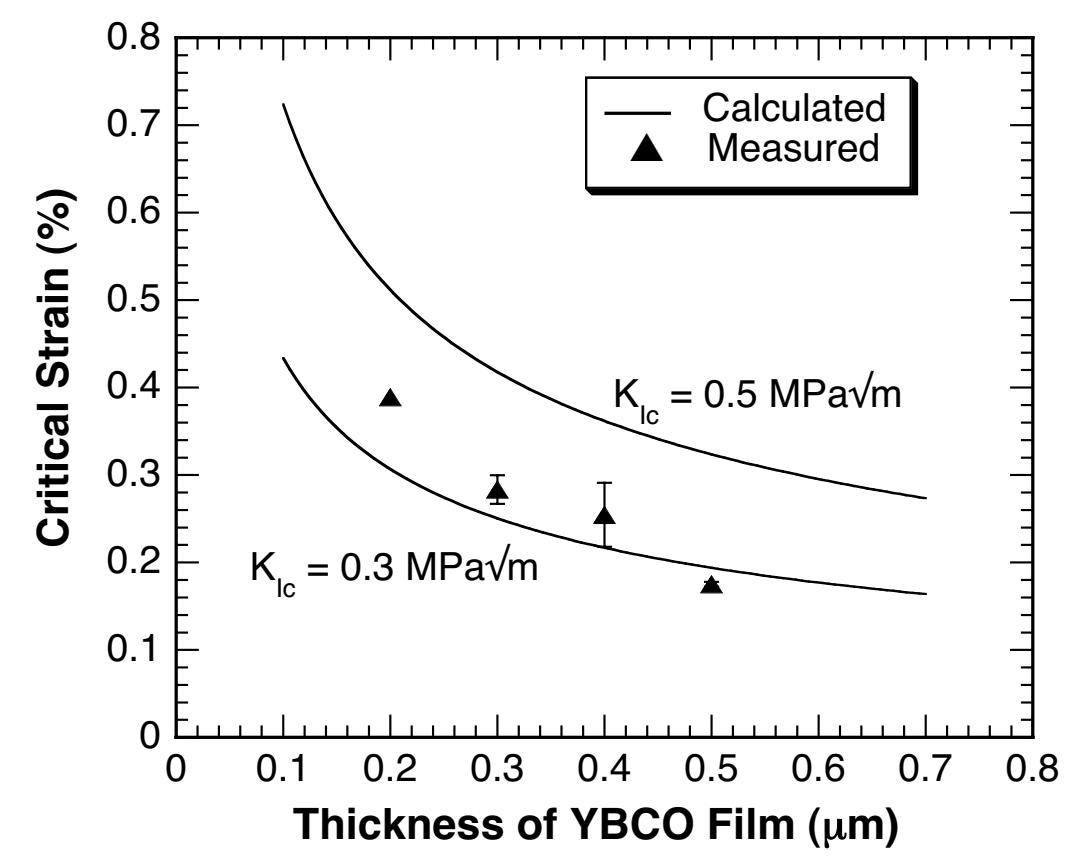

Fig. 66 Measured critical strain $\left(\varepsilon_{c r}\right)$ vs. YBCO layer thickness. Calculated critical strain values based on analytical prediction are also shown. 


\subsection{6.b Residual Stress Evaluation}

Residual strains, and hence residual stresses, develop during processing of coated conductors due to differences in thermal expansion for the various layers. Additional intrinsic stresses caused by film growth may also contribute to the residual stresses. These stresses may cause microcracking, which may cause deterioration of the superconductor. Therefore, it is critical to evaluate residual stresses in coated conductors and improve our understanding of the relationship between residual stresses and various processing parameters.

Previously, we evaluated the residual stresses in thin films with a bilayer configuration using both an X-ray technique and curvature measurements based on 3-D optical interferometry. Specifically, we evaluated residual stresses in $\mathrm{ZrO}_{2}$ on Hastelloy $\mathrm{C}(\mathrm{HC})$ and $\mathrm{YBCO}$ films on $\mathrm{LaAlO}_{3}$ substrates. Currently, we are extending our measurements to coated conductors with the standard ISD architecture: $\mathrm{YBCO} / \mathrm{CeO}_{2} / \mathrm{YSZ} / \mathrm{HE}-\mathrm{MgO} / \mathrm{ISD}-\mathrm{MgO} / \mathrm{HC}$. In order to measure and predict residual stresses in the superconductor layer, residual stresses in the buffer layers must be known. To this end, we initiated the measurement of residual stress in the individual layers of the multi-layer conductor. Stresses were evaluated by 3-D optical interferometry, which measures the change in substrate curvature that results from depositing the layer of interest. With the measured changes in curvature, stresses in the individual layers are estimated using a modified form of Stoney's equation[10].

First, measurements were made to determine residual stresses in ISD MgO that was deposited on an HC substrate at room temperature. Prior to depositing the $\mathrm{MgO}$ layer, the $\mathrm{HC}$ substrate (thickness $\approx 150 \mu \mathrm{m}$ ) was polished to a $1 \mu \mathrm{m}$ surface finish and its curvature was measured by optical interferometry. The $\mathrm{MgO}$ layer (thickness $\approx 2 \mu \mathrm{m}$ ) was then deposited on the HC substrate at room temperature by the ISD process, and the resulting substrate curvature was measured again. Using the difference in curvature before and after the deposition of $\mathrm{MgO}$, the average residual stress was estimated to be $50 \mathrm{MPa}$. Because $\mathrm{MgO}$ was deposited at room temperature, the measured residual stress in this case is believed to be the contribution of the intrinsic stress developed during the film growth. Currently, effort is in progress to deposit the remaining buffer layers and estimate their residual stresses.

\subsection{Bi-Pb-Sr-Ca-Cu-O Conductor}

\subsubsection{Modifications to Thermal Slide Heat Treatment of Bi-2223 Conductors}

In a series of recent publications [11-13], we reported on extensive work with American Superconductor (AMSC) to develop a new heat treatment 
paradigm for the $\mathrm{Ag} / \mathrm{Bi}-2223$ composite conductor. This new paradigm, called thermal slide heat treatment (TSHT) and described in [12], incorporates systematic variations of the oxygen partial pressure $\left(\mathrm{pO}_{2}\right)$ and temperature $(\mathrm{T})$ into the first heat treatment (HT-1) of Ag/Bi-2223 composite wire. The general nature of these variations relative to a conventional single $\mathrm{pO}_{2} / \mathrm{T}$ HT- 1 is indicated in Figure 67. The purpose of the TSHT approach to HT-1 is to reduce the size of non-superconducting second phases (NSPs) at the end of HT-1, i.e., just prior to the intermediate size reduction (ISR) that is done to redensify the Bi2223 filament cores.

Five-step TSHT-type HT-1
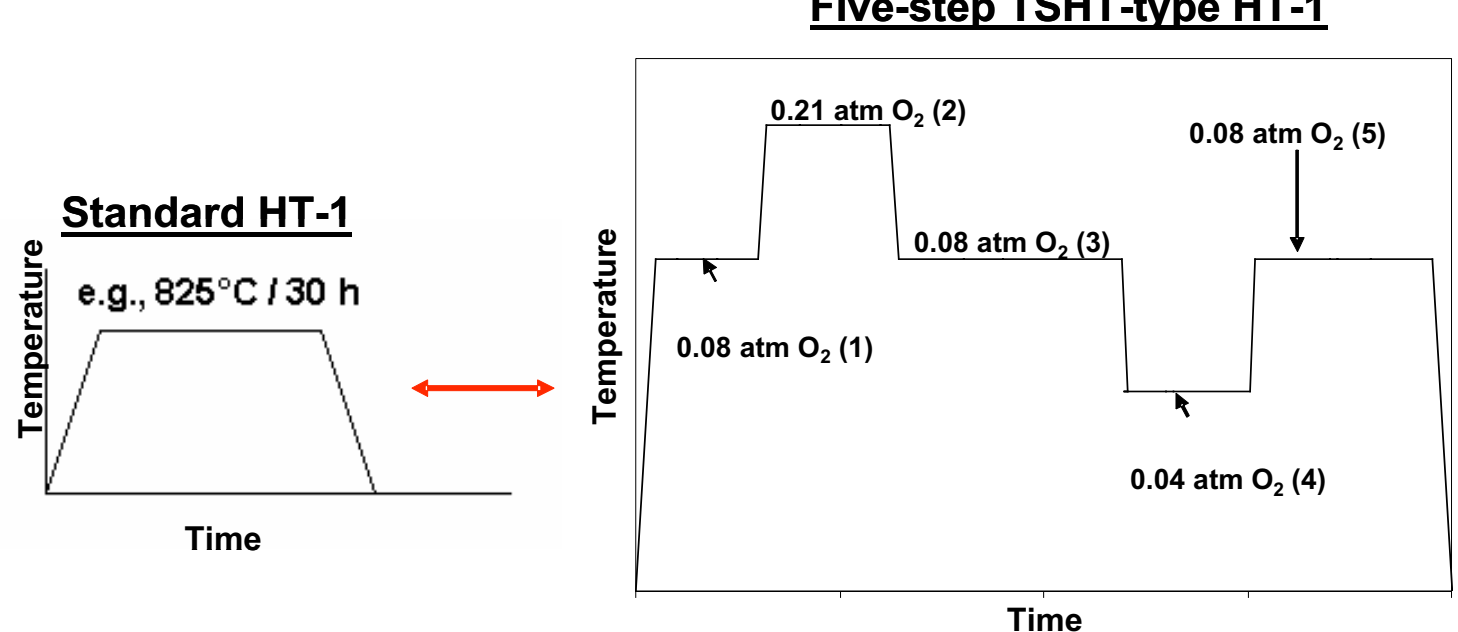

Time

Fig. 67 Schematic representations of the standard AMSC HT-1 and the fivestep TSHT-type HT-1.

Reducing the size of NSPs is important to reduce the damage done to soft Bi-2223 grain colonies by hard NSPs--mainly alkaline earth cuprates (AECs)-during the ISR rolling operation. As we reported in [12], there is a clear correlation between the volumetric concentration of large NSPs (i.e., NSP particles $>1 \mu \mathrm{m}^{2}$ ) after HT- 1 and the $\mathrm{J}_{\mathrm{c}}$ of the fully processed wire. From the work reported in [12] and [13], we showed that the TSHT approach significantly reduces the number of large NSPs after HT-1, which leads to fully processed $\mathrm{Ag} / \mathrm{Bi}-2223$ wire with measurably higher $\mathrm{J}_{\mathrm{C}}$ values [12]. A summary comparison of the results of electron microscopy and electrical performance measurements is given in Table 2. The parameter $\mathrm{J}_{\mathrm{c}}(\mathrm{TSHT}) / \mathrm{J}_{\mathrm{c}}$ (STD.) is the ratio of $\mathrm{J}_{\mathrm{c}}$ for fully processed wire specimens that received a TSHT-type HT-1 to the $\mathrm{J}_{\mathrm{c}}$ for specimens from the same batch of wire that received the standard AMSC HT-1. Our experience with TSHT-treated specimens has consistently shown that they produce a post HT-1 microstructure with smaller sized NSPs both on an areaaveraged basis and in terms of the largest ones that actually form. 
Table 2. Summary of processing and performance results for a series of Ag/Bi-2223 wires that received AMSC standard HT-1s and Base Case (BC) TSHT type HT-1s. The NSP area percent and particle size parameters were measured after HT-1.

\begin{tabular}{|l|c|c|c|c|c|}
\hline HT-1 Type & $\begin{array}{c}\text { Avg. Area } \\
\text { Percent NSP }\end{array}$ & $\begin{array}{c}\text { Largest } \\
\text { NSP }\end{array}$ & $\begin{array}{c}90 \% \text { of } \\
\text { Particles }\end{array}$ & NSP mix & $\frac{\text { Jc(TSHT) }}{\text { Jc(STD.) }}$ \\
\hline AMSC STD. & $>12 \%$ & $>30$ sq. $\mu \mathrm{m}$ & $\leq 13$ sq. $\mu \mathrm{m}$ & $2 / 1$ and $14 / 24$ AEC & - \\
BC TSHT & $<8 \%$ & $<10$ sq. $\mu \mathrm{m}$ & $\leq 4$ sq. $\mu \mathrm{m}$ & $2 / 1$ and $14 / 24$ AEC & 1.13 \\
\hline
\end{tabular}

Subsequent investigation of the TSHT approach with other AMSC production wire batches has indicated that additional improvements in terms of processing costs and performance may be possible if the extent of conversion of Bi-2212 in the precursor powder to Bi-2223 during HT-1 can be reduced. In recent studies we have focused on modified TSHTs with shorter total heat treatment times, wherein the time reductions were achieved by reducing the length of time spent at the high conversion rate condition of 0.08 atm $\mathrm{O}_{2} / 825^{\circ} \mathrm{C}$. Specifically, the duration of Steps 3 and 5 in Fig. 67 were reduced to varying extents. The results of a recently completed series of studies of this type are summarized in Table 3. As expected, shortening the overall time of the TSHT process led to reduced conversion of $\mathrm{Bi}-2212$ to $\mathrm{Bi}-2223$, thus providing more potential for phase evolution and liquid phase development during the final heat treatment. Also noteworthy is the observation that the $\mathrm{J}_{c}(\mathrm{TSHT}) / \mathrm{J}_{\mathrm{c}}$ (STD.) ratio maintained the levels obtained with the 2800 minute base case TSHT HT-1 down to TSHT HT-1 treatment times of 1400 minutes.

Table 3. Summary of processing and performance results for a series of Ag/Bi-2223 wires that received modified TSHT-type HT-1s. The \%Bi-2223 after HT-1 is determined by the ratio of the intensity of the (0010) diffraction line of Bi-2223 to the sum of the intensities of the Bi-2223 (0010) and Bi-2212 (008) diffraction lines [12].

\begin{tabular}{|c|c|c|c|c|c|}
\hline Parameter & $\begin{array}{c}\text { Base Case } \\
\text { TSHT }\end{array}$ & $\begin{array}{c}\text { MOD-01 } \\
\text { TSHT }\end{array}$ & $\begin{array}{c}\text { MOD-02 } \\
\text { TSHT }\end{array}$ & $\begin{array}{c}\text { MOD-03 } \\
\text { TSHT }\end{array}$ & $\begin{array}{c}\text { MOD-04 } \\
\text { TSHT }\end{array}$ \\
\hline Total Time (minutes) & 2800 & 2100 & 1400 & 1100 & 1200 \\
$\%$ \%i-2223 after HT-1 & $95+$ & ca. 94 & ca. 88 & ca. 77 & ca. 83 \\
Jc(TSHT)/Jc(STD.) & 1.13 & 1.13 & 1.14 & 1.07 & 1.02 \\
\hline
\end{tabular}




\section{References}

1. Practical Superconductor Development for Electrical Power Applications, Argonne National Laboratory, Annual Report for FY 2001.

2. Practical Superconductor Development for Electrical Power Applications, Argonne National Laboratory, Quarterly Report for Jan.-Mar., 2002.

3. Practical Superconductor Development for Electrical Power Applications, Argonne National Laboratory, Annual Report for FY 2002.

4. J. D. Budai, R. T. Young, B. S. Chao, Appl. Phys. Lett. 62, (15), 1836-1838 (1993).

5. M. Zhou, H. Guo, D.M. Liu, T.Y. Zuo, L.H. Zhai, Y.L. Zhou, R.P. Wang, S.H. Pan, H.H. Wang, Physica C, 337, 101-105 (2000).

6. B. W. Kang, A. Goyal, D. F. Lee, J. E. Mathis, E. D. Specht, P. M. Martin, and D. M. Kroeger, J. Mater. Res. 17, 1750 (2002).

7. J. R. Ferraro and V. A. Maroni, Applied Spectroscopy 44, 351-366 (1990).

8. M. N. Iliev, American Chemical Society Symposium Series 730, 107-119 (1999).

9. G. Gibson, L. F. Cohen, R. G. Humphreys, and J. L. MacManus-Driscoll, Physica C 333, 139-145 (2000).

10. G. Stoney, Proc. R. Soc. London, Ser. A 82, 172 (1909).

11. Y.L. Tang, D.J. Miller, R.M. Baurceanu, V.A. Maroni, and R.D. Parrella, Supercond. Sci. Technol. 15, 1365-1371 (2002).

12. R.M. Baurceanu, V.A. Maroni, N. N. Merchant, A.K. Fischer, M.J. McNallan, and R.D. Parrella, Supercond. Sci. Technol. 15, 1167-1175 (2002).

13. R.M. Baurceanu, V.A. Maroni, N. N. Merchant, A.K. Fischer, M.J. McNallan, and R.D. Parrella, Supercond. Sci. Technol. 15, 1160-1166 (2002). 


\section{Patents 2000-2003}

Fabrication of High Temperature Superconductors

Uthamalingam Balachandran, Stephen E. Dorris, Beihai Ma, and Meiya Li

U.S. Patent No. 6,579,360 B2 (June 17, 2003).

Metallic Substrates for High-Temperature Superconductors

T. Truchan, D. Miller, K. C. Goretta, U. Balachandran, and R. Foley (U. of IL)

U.S. Patent No. 6,455,166 (Sept. 24, 2002).

Method for Preparing High-Temperature Superconductor

Uthamalingam Balachandran and Michael P. Chudzik

U.S. Patent No. 6,361,598 (March 26, 2002).

Shielded High- $\mathrm{T}_{\mathrm{C}}$ BSCCO Tapes or Wires for High-Field Applications

Uthamalingam Balachandran, Milan Lelovic, and Nicholas G. Eror

U.S. Patent No. 6,252,096 (June 26, 2001); U.S. Patent 6,466,805 (Oct. 15, 2002).

Thermomechanical Means to Improve Critical Current Density of BSCCO Tapes Uthamalingam Balachandran, Roger B. Poeppel, Pradeep Haldar (IGC), and Lesizek Motowidlo (IGC), U.S. Patent 6,240,619 (June 5, 2001).

Method of Manufacturing a High-Temperature Superconductor with Improved Transport Properties

Uthamalingam Balachandran, Richard Siegel, and Thomas Askew

U.S. Patent No. 6,191,075 (February 20, 2001).

Bearing Design for Flywheel Energy Storage Using High- $\mathrm{T}_{\mathrm{c}}$ Superconductors John R. Hull and Thomas M. Mulcahy U.S. Patent No. 6,153,958 (Nov. 28, 2000).

Method and Apparatus for Measuring Gravitational Acceleration Utilizing a High- Temperature Superconducting Bearing

John R. Hull

U.S. Patent 6,079,267 (June 27, 2000).

Improvements in Levitation Pressure and Friction Losses in Superconducting Bearings,

John R. Hull

U.S. Patent 6,175,175 (January 16, 2001).

Trapped Field Internal Dipole Superconducting Motor/Generator John R. Hull

U.S. Patent 6,169,352 (January 2, 2001). 
Reluctance Apparatus for Flywheel Energy Storage

John R. Hull

U.S. Patent 6,097,118 (August 1, 2000).

Large Area Bulk Superconductors

Dean J. Miller and Michael B. Field

U.S. Patent 6,410,487 (June 25, 2002).

\section{Publications: FY 2003}

R. M. Baurceanu, V. A. Maroni, N. M. Merchant, A. K. Fischer, M. J. McNallan, and R. D. Parella, Investigation of a Multi-setpoint First Heat Treatment Methodology for the Silver-Sheathed ( $\mathrm{Bi}, \mathrm{Pb})_{2} \mathrm{Sr}_{2} \mathrm{Ca}_{2} \mathrm{Cu}_{3} \mathrm{O}_{\mathrm{x}}$ Composite Conductor, Superconductor Science and Technology, 15, 1167-1175 (2002).

R. M. Baurceanu, V. A. Maroni, N. M. Merchant, A. K. Fischer, M. J. McNallan, and R. D. Parella, Time Evolution of Phase Composition and Microstructure in the Ag/Bi-2223 Composite Superconductor Heat-treated at Specific $\mathrm{pO}_{2} /$ Temperature Setpoints, Superconductor Science and Technology, 15, 11601166 (2002).

V. A. Maroni, K. Venkataraman, A. J. Kropf, C. U. Segre, Y. Huang, G. N. Riley, Jr., Nondestructive Analysis of Phase Evolution and Microstructure Development in $\mathrm{Ag} /(\mathrm{Bi}, \mathrm{Pb})_{2} \mathrm{Sr}_{2} \mathrm{Ca}_{2} \mathrm{Cu}_{3} \mathrm{O}_{\mathrm{x}}$ Composite Conductor by $25 \mathrm{keV}$ Transmission X-ray Diffraction, Physica C, 382 , 21-26 (2002).

Y. L. Tang, D. J. Miller, R. M. Baurceanu, V. A. Maroni, and R. D. Parella, Improved Microstructure in Ag/Bi-2223 Composite Tapes by Systematic Variation of Heat Treatment Parameters, Superconductor Science and Technology, 15, 1365-1371 (2002).

J. H. Je (POSTECH), H. You, W. G. Cullen, V. A. Maroni, B. Ma, R. E. Koritala, and C. Thieme (American Superconductor), Detection of a Pd-Ni Interlayer at the $\mathrm{Pd} / \mathrm{Ni}$ Interface of an Epitaxial Pd Film on Cube Textured Nickel (001), Physica C 383 (2002) 241-246.

M. Li, B. Ma, R. E. Koritala, B. L. Fisher, K. Venkataraman, and U. Balachandran, Pulsed Laser Deposition of YBCO Thin Films on IBAD-YSZ Substrates, Supercond. Sci. Technol. 16 (2003) 105-109. 
B. Ma, M. Li, R. E. Koritala, B. L. Fisher, A. R. Markowitz, R. A. Erck, R. Baurceanu, S. E. Dorris, D. J. Miller, and U. Balachandran, Pulsed Laser Deposition of YBCO Films on ISD MgO Buffered Metal Tapes, Supercond. Sci. Technol. 16 (2003) 464-472.

M. Li, B. Ma, R. E. Koritala, B. L. Fisher, K. Venkataraman, V. A. Maroni, V. Vlasko-Vlasov, P. Berghuis, U. Welp, K. E. Gray, and U. Balachandran, Pulsed Laser Deposition of c-Axis Untilted YBCO Films on c-Axis Tilted ISD MgOBuffered Metallic Substrates, Physica C 387 (2003) 373-381.

U. Balachandran, B. Ma, M. Li, B. L. Fisher, R. E. Koritala, D. J. Miller, and S. E. Dorris, Development of Coated Conductors by Inclined Substrate Deposition, Physica C 392-396 (2003) 806-814.

S. Tsukui, R. E. Koritala, M. Li, K. C. Goretta, M. Adachi, J. E. Baker, and J. L. Routbort, Oxygen and Cation Diffusion in YBCO Coated Conductors, Physica C 392-396 (2003) 841-846.

H. Claus, B. Ma, A. P. Paulikas, Y. Tang, R. Nikolova, B. W. Veal, and K. E. Gray, Oxygen Doping of Grain Boundaries in $\mathrm{YBa}_{2} \mathrm{Cu}_{3} \mathrm{O}_{\mathrm{x}}$ Bicrystal Films, Proc. 6th European Conf. on Applied Superconductivity, Sorrento, Napoli, Italy, Sept. 1418, 2003.

K. Venkataraman, A. J. Kropf, C. U. Segre, Q. X. Jia, A. Goyal, B. W. Kang, S. Chattopadhyay, H. You, V. A. Maroni, Detection of Interfacial Strain and Phase Separation in $\mathrm{MBa}_{2} \mathrm{Cu}_{3} \mathrm{O}_{7-x}$ Thin Films Using Raman Spectroscopy and X-ray Diffraction Space Mapping, Physica C 402, 1-16 (2004).

V. K. Vlasko-Vlasov, H. Claus, U. Welp, K. E. Gray (MSD), B. Ma, and U. Balachandran, Improving Ratio of the Grain Boundary and Bulk Critical Currents in $\mathrm{YBa}_{2} \mathrm{Cu}_{3} \mathrm{O}_{7-\delta}$ Films, Appl. Phys. Lett., 84(2), 242-244 (2004).

\section{$\underline{\text { Submitted }}$}

J. R. Hull and M. Murakami, Applications of Bulk High-Temperature Superconductors, submitted for publication as an invited contribution to Proc. IEEE (Sept 2003). 


\section{Distribution for ANL-04/19}

Internal:
U. Balachandran
Y.S. Cha
G. Crabtree
S. E. Dorris
H. Drucker
B. L. Fisher
K. E. Gray

J. Harmon

R. B. Poeppel

J. R. Hull

S. Lake

B. Ma

V. A. Maroni

D. J. Miller

J. J. Picciolo

A. C. Raptis

W. J. Shack

W. W. Schertz

J. P. Singh

A. M. Wolsky

TIS Files

External:

DOE-OSTI (2)

ANL-E Library

ANL-W Library

U.S. Department of Energy, Washington:

Office of Electric Transmission and Distribution:

W. Parks

J. Daley

R. George

M. Reed

H. Chhabra

Basic Energy Sciences-Materials Science:

T. J. Fitzsimmons

R. Gottschall

B. Strauss

A. Dragoo

Energy Technology Division Review Committee:

M. L. Corradini, University of Wisconsin

J. C. Davis, LLNL (retired)

J. M. Deutch, MIT

N. G. Eror, University of Pittsburgh

C. Logan, Industrial Quality, Inc.

M. A. Ulrickson, Sandia National Laboratory

R. Zoughi, University of Missouri-Rolla 
Other - Industry - University

S. Ahmed, Southern California Edison, Rosemead, CA

J. Badin, Energetics, Inc., Columbia, MD

P. Barnes, Air Force Research Laboratory, Wright-Patterson Air Force Base

P. Berdahl, E. O. Lawrence Berkeley National Laboratory

R. Bhattacharya, National Renewable Energy Laboratory

R. S. Bhattacharya, UES, Inc., Dayton, OH

P. Chu, University of Houston

P. Clem, Sandia National Laboratories

C. Cox, Bob Lawrence and Associates, Inc., Alexandria, VA

A. Day, Boeing Corp., Seattle, WA

D. Driscoll, Rockwell Automation, Cleveland, $\mathrm{OH}$

J. Ekin, National Institute of Standards and Technology, Boulder, CO

P. Grant, EPRI, Palo Alto, CA

D. Gubser, Naval Research Laboratory

P. Haldar, University at Albany, State University of New York

R. Hammond, Laboratory for Advanced Materials, Stanford University

R. A. Hawsey, Oak Ridge National Laboratory

P. Herz, TMS, Inc., Gaithersburg, MD

R. L. Hughey, Southwire Co., Carrollton, GA

Y. Iwasa, Francis Bitter National Magnet Lab., Massachusetts Institute of Technology

M. Justiniano, Energetics, Inc., Columbia, MD

D. C. Larbalestier, University of Wisconsin - Madison

M. Levine, E. O. Lawrence Berkeley National Laboratory

A. Malozemoff, American Superconductor Corp., Westborough, MA

K. Marken, Oxford Instruments, Inc., Carteret, NJ

B. McCallum, Ames Laboratory, Ames, IA

S. Mehta, Waukesha Electric Systems, Waukesha, WI

M. W. Morgan, Ability Engineering Technology, Inc., South Holland, IL

C. Oberly, Wright Air Force Laboratory, Dayton, $\mathrm{OH}$

P. Pellegrino, IGC-SuperPower, Schenectady, NY

D. Peterson, Los Alamos National Laboratory

C. H. Rosner, CardioMag Imaging, Inc., Schenectady, NY

R. Russo, Lawrence Berkeley National Laboratory

H. Schneider-Muntan, National High Magnetic Field Laboratory, Tallahassee, FL

J. Schwartz, Florida State University

V. Selvamanickam, IGC-SuperPower, Schenectady, NY

M. Strasik, Boeing Corp., Seattle

M. Suenaga, Brookhaven National Laboratory

H. Weinstock, Air Force Office of Scientific Research, Arlington, VA

D. O. Welch, Brookhaven National Laboratory

W. Wong-Ng, NIST 